\title{
Completely Integrable Systems Associated with Classical Root Systems
}

Toshio OSHIMA

Graduate School of Mathematical Sciences, University of Tokyo, 3-8-1, Komaba, Meguro-ku, Tokyo 153-8914, Japan

E-mail: oshima@ms.u-tokyo.ac.jp

URL: http://akagi.ms.u-tokyo.ac.jp/

Received December 14, 2006, in final form March 19, 2007; Published online April 25, 2007

Original article is available at http://www.emis.de/journals/SIGMA/2007/061/

\begin{abstract}
We study integrals of completely integrable quantum systems associated with classical root systems. We review integrals of the systems invariant under the corresponding Weyl group and as their limits we construct enough integrals of the non-invariant systems, which include systems whose complete integrability will be first established in this paper. We also present a conjecture claiming that the quantum systems with enough integrals given in this note coincide with the systems that have the integrals with constant principal symbols corresponding to the homogeneous generators of the $B_{n}$-invariants. We review conditions supporting the conjecture and give a new condition assuring it.
\end{abstract}

Key words: completely integrable systems; Calogero-Moser systems; Toda lattices with boundary conditions

2000 Mathematics Subject Classification: 81R12; 70H06

To the memory of Professor Vadim B. Kuznetsov

\section{Introduction}

A Schrödinger operator

$$
P=-\frac{1}{2} \sum_{j=1}^{n} \frac{\partial^{2}}{\partial x_{j}^{2}}+R(x)
$$

with the potential function $R(x)$ of $n$ variables $x=\left(x_{1}, \ldots, x_{n}\right)$ is called completely integrable if there exist $n$ differential operators $P_{1}, \ldots, P_{n}$ such that

$$
\begin{aligned}
& {\left[P_{i}, P_{j}\right]=0 \quad(1 \leq i<j \leq n)} \\
& P \in \mathbb{C}\left[P_{1}, \ldots, P_{n}\right] \\
& P_{1}, \ldots, P_{n} \quad \text { are algebraically independent. }
\end{aligned}
$$

In this paper, we explicitly construct the integrals $P_{1}, \ldots, P_{n}$ for completely integrable potential functions $R(x)$ of the form

$$
R(x)=\sum_{1 \leq i<j \leq n}\left(u_{i j}^{-}\left(x_{i}-x_{j}\right)+u_{i j}^{+}\left(x_{i}+x_{j}\right)\right)+\sum_{k=1}^{n} v_{k}\left(x_{k}\right)
$$

${ }^{\star}$ This paper is a contribution to the Vadim Kuznetsov Memorial Issue 'Integrable Systems and Related Topics'. The full collection is available at http://www.emis.de/journals/SIGMA/kuznetsov.html 
appearing in other papers. The Schrödinger operators with these commuting differential operators treated in this paper include Calogero-Moser-Sutherland systems (cf. [5, 22, 27, 28, 36]), Heckman-Opdam's hypergeometric systems (cf. [34] for type $A_{n-1}$, [11] in general), their extensions (cf. $[1,8,14,23,24,25,30])$ and finite Toda lattices corresponding to (extended) Dynkin diagrams for classical root systems (cf. [2, 9, 10, 17, 26, 33, 38]) and those with boundary conditions (cf. $[8,13,18,19,20,21,23,30])$.

Put $\partial_{j}=\partial / \partial x_{j}$ for simplicity. We denote by $\sigma(Q)$ the principal symbol of a differential operator of $Q$. For example, $\sigma(P)=-(1 / 2)\left(\xi_{1}^{2}+\cdots+\xi_{n}^{2}\right)$.

We note that [40] proves that the potential function is of the form (1.3) if

$$
\sigma\left(P_{k}\right)=\sum_{1 \leq j_{1}<\cdots<j_{k} \leq n} \xi_{j_{1}}^{2} \cdots \xi_{j_{k}}^{2} \quad \text { for } \quad k=1, \ldots, n .
$$

In this case we say that $R(x)$ is an integrable potential function of type $B_{n}$ or of the classical type. Moreover when $R(x)$ is symmetric with respect to the coordinate $\left(x_{1}, \ldots, x_{n}\right)$ and invariant under the coordinate transformation $\left(x_{1}, x_{2}, \ldots, x_{n}\right) \mapsto\left(-x_{1}, x_{2}, \ldots, x_{n}\right)$, then $R(x)$ is determined by [32] for $n \geq 3$ and by [24] for $n=2$ and $P_{k}$ are calculated by [29].

Classifications of the integrable potential functions under certain conditions are given in $[23,24,25,30,37,40]$ etc. In Section 9 we review them and we present Conjecture which claims that the potential functions given in this note exhaust those of the completely integrable systems satisfying (1.4). We also give a new condition which assures Conjecture.

If $v_{k}=0$ for $k=1, \ldots, n$, we can expect $\sigma\left(P_{k}\right)=\sum_{1 \leq j_{1}<\cdots<j_{k} \leq n} \xi_{j_{1}}^{2} \cdots \xi_{j_{k}}^{2}$ for $k=1, \ldots, n-1$ and $\sigma\left(P_{n}\right)=\xi_{1} \xi_{2} \cdots \xi_{n}$ and we say the integrable potential function is of type $D_{n}$. If $v_{k}=0$ and $u_{i j}^{+}=0$ for $k=1, \ldots, n$ and $1 \leq i<j \leq n$, we can expect $P_{1}=\partial_{1}+\cdots+\partial_{n}, \sigma\left(P_{k}\right)=$ $\sum_{1 \leq j_{1}<\cdots<j_{k} \leq n} \xi_{j_{1}} \cdots \xi_{j_{k}}$ for $k=2, \ldots, n$ and we say that the integrable potential function is of type $A_{n-1}$. Note that the integrable potential function of type $A_{n-1}$ or $D_{n}$ is of type $B_{n}$.

The elliptic potential function of type $A_{n-1}$ with

$$
u_{i j}^{-}(t)=C \wp\left(t ; 2 \omega_{1}, 2 \omega_{2}\right)+C^{\prime}, \quad u_{i j}^{+}(t)=v_{k}(t)=0 \quad\left(C, C^{\prime} \in \mathbb{C}\right)
$$

(cf. [28]) and that of type $B_{n}$ with

$$
\begin{aligned}
& u_{i j}^{-}(t)=v_{i j}^{+}(t)=A \wp\left(t ; 2 \omega_{1}, 2 \omega_{2}\right), \\
& v_{k}(t)=\sum_{j=0}^{3} C_{j} \wp\left(t+\omega_{j} ; 2 \omega_{1}, 2 \omega_{2}\right)-\frac{C}{2}, \quad\left(A, C_{i}, C \in \mathbb{C}\right)
\end{aligned}
$$

introduced by [12] are most fundamental and their integrability and the integrals of higher order are established by $[25,29,32]$. Here $\wp\left(t ; 2 \omega_{1}, 2 \omega_{2}\right)$ is the Weierstrass elliptic function whose fundamental periods are $2 \omega_{1}$ and $2 \omega_{2}$ and

$$
\omega_{0}=0, \quad \omega_{1}+\omega_{2}+\omega_{3}=0 .
$$

Other potential functions are suitable limits of these elliptic potential functions, which is shown in $[8,13,33]$ etc. We will study integrable systems by taking analytic continuations of the integrals given in [25] with respect to a suitable parameter, which is done for the invariant systems (of type $A_{n-1}$ ) by [32] and (of types $B_{n}$ and $D_{n}$ ) by [29] and for the systems of type $A_{n-1}$ by [33]. The main purpose of this note is to give the explicit expression of the operators $P_{1}, \ldots, P_{n}$ in (1.2) in this unified way. Namely we construct enough commuting integrals of the non-invariant systems from those of the invariant systems given by [24, 29, 32]. Such study of the systems of types $A_{n-1}, B_{2}, B_{n}(n \geq 3)$ and $D_{n}$ are explained in Sections 3, 4, 5, 6, respectively. 
Since the integrals of the system of type $A_{n-1}$ are much simpler than those of type $B_{n}$, we review the above analytic continuation for the systems of type $A_{n-1}$ in Section 3 preceding to the study for the systems of type $B_{n}$. There are many series of completely integrable systems of type $B_{2}$, which we review and classify in Section 4 with taking account of the above unified way.

We present 8 series of potential functions of type $B_{n}$ in Section 5 . There are 3 (elliptic, trigonometric or hyperbolic and rational) series of the invariant potentials of type $B_{n}$ whose enough integrals are constructed by [25] and [29]. The complete integrability of the remaining 5 series of the potential functions is shown in Section 5, which is conjectured by [8] (4 series), partially proved by $[18,19,21]$ (3 series) and announced by [30] (5 series). The complete integrability of two series among them seems to be first established in this note. Note that when $n \geq 3$, our systems which do not belong to these 8 series of type $B_{n}$ are the CalogeroMoser systems with elliptic potentials and the finite Toda lattices of type $A_{n-1}^{(1)}$, whose complete integrability is known.

The main purpose of our previous study in [23, 24, 25, 30] is classification of the completely integrable systems associated with classical root systems. In this note we explicitly give integrals of all the systems classified in our previous study with reviewing known integrals.

Since our expression of $P_{k}$ is natural, we can easily define their classical limits without any ambiguity and get completely integrable Hamiltonians of dynamical systems together with their enough integrals. This is clarified in Section 7.

In Section 8 we examine ordinary differential operators which are analogues of the Schrödinger operators studied in this note.

\section{Notation and preliminary results}

Let $\left\{e_{1}, \ldots, e_{n}\right\}$ be the natural orthonormal base of the Euclidean space $\mathbb{R}^{n}$ with the inner product

$$
\langle x, y\rangle=\sum_{j=1}^{n} x_{j} y_{j} \quad \text { for } \quad x=\left(x_{1}, \ldots, x_{n}\right), y=\left(y_{1}, \ldots, y_{n}\right) \in \mathbb{R}^{n}
$$

Here $e_{j}=\left(\delta_{1 j}, \ldots, \delta_{n j}\right) \in \mathbb{R}^{n}$ with Kronecker's delta $\delta_{i j}$.

Let $\alpha \in \mathbb{R}^{n} \backslash\{0\}$. The reflection $w_{\alpha}$ with respect to $\alpha$ is a linear transformation of $\mathbb{R}^{n}$ defined by $w_{\alpha}(x)=x-\frac{2\langle\alpha, x\rangle}{\langle\alpha, \alpha\rangle} \alpha$ for $x \in \mathbb{R}^{n}$. Furthermore we define a differential operator $\partial_{\alpha}$ by

$$
\left(\partial_{\alpha} \varphi\right)(x)=\left.\frac{d}{d t} \varphi(x+t \alpha)\right|_{t=0}
$$

and then $\partial_{j}=\partial_{e_{j}}$.

The root system $\Sigma\left(B_{n}\right)$ of type $B_{n}$ is realized in $\mathbb{R}^{n}$ by

$$
\begin{aligned}
& \Sigma\left(A_{n-1}\right)^{+}=\left\{e_{i}-e_{j} ; 1 \leq i<j \leq n\right\} \\
& \Sigma\left(D_{n}\right)^{+}=\left\{e_{i} \pm e_{j} ; 1 \leq i<j \leq n\right\} \\
& \Sigma\left(B_{n}\right)_{S}^{+}=\left\{e_{k} ; 1 \leq k \leq n\right\} \\
& \Sigma\left(B_{n}\right)^{+}=\Sigma\left(D_{n}\right)^{+} \cup \Sigma\left(B_{n}\right)_{S}^{+}, \quad \text { for } \quad F=A_{n-1}, \quad D_{n} \quad \text { or } \quad B_{n} . \\
& \Sigma(F)=\left\{\alpha,-\alpha ; \alpha \in \Sigma(F)^{+}\right\} \quad \text {. }
\end{aligned}
$$

The Weyl groups $W\left(B_{n}\right)$ of type $B_{n}, W\left(D_{n}\right)$ of type $D_{n}$ and $W\left(A_{n-1}\right)$ of type $A_{n-1}$ are the groups generated by $w_{\alpha}$ for $\alpha \in \Sigma\left(B_{n}\right), \Sigma\left(D_{n}\right)$ and $\Sigma\left(A_{n-1}\right)$, respectively. The Weyl group 
$W\left(A_{n-1}\right)$ is naturally identified with the permutation group $\mathfrak{S}_{n}$ of the set $\{1, \ldots, n\}$ with $n$ elements. Let $\epsilon$ be the group homomorphism of $W\left(B_{n}\right)$ defined by

$$
\epsilon(w)=\left\{\begin{aligned}
1 & \text { if } \quad w \in W\left(D_{n}\right), \\
-1 & \text { if } \quad w \in W\left(B_{n}\right) \backslash W\left(D_{n}\right) .
\end{aligned}\right.
$$

The potential function (1.3) is of the form

$$
R(x)=\sum_{\alpha \in \Sigma\left(D_{n}\right)^{+}} u_{\alpha}(\langle\alpha, x\rangle)+\sum_{\alpha \in \Sigma\left(B_{n}\right)_{S}^{+}} v_{\beta}(\langle\beta, x\rangle)
$$

with functions $u_{\alpha}$ and $v_{\beta}$ of one variable. For simplicity we will denote

$$
\begin{aligned}
& u_{\alpha}(x)=u_{-\alpha}(x)=u_{\alpha}(\langle\alpha, x\rangle) \quad \text { for } \quad \alpha \in \Sigma\left(D_{n}\right)^{+}, \\
& v_{\beta}(x)=v_{-\beta}(x)=v_{\beta}(\langle\beta, x\rangle) \quad \text { for } \quad \beta \in \Sigma\left(B_{n}\right)_{S}^{+}, \\
& u_{i j}^{ \pm}(x)=u_{e_{i} \pm e_{j}}(x), \quad v_{k}(x)=v_{e_{k}}(x) .
\end{aligned}
$$

Lemma 1. For a bounded open subset $U$ of $\mathbb{C}$, there exists an open neighborhood $V$ of 0 in $\mathbb{C}$ such that the following statements hold.

i) The function $\lambda \sinh ^{-1} \lambda z$ is holomorphically extended to $(z, \lambda) \in(U \backslash\{0\}) \times V$ and the function is $1 / z$ when $\lambda=0$.

ii) Suppose $\operatorname{Re} \lambda>0$. Then the functions

$$
e^{2 \lambda t} \sinh ^{-2} \lambda(z \pm t) \quad \text { and } \quad e^{4 \lambda t}\left(\sinh ^{-2} \lambda(z \pm t)-\cosh ^{-2} \lambda(z \pm t)\right)
$$

are holomorphically extended to $(z, q) \in U \times V$ with $q=e^{-2 \lambda t}$ and the functions are $4 e^{\mp 2 \lambda z}$ and $16 e^{\mp 4 \lambda z}$, respectively, when $q=0$.

Proof. The claims are clear from

$$
\begin{aligned}
& \lambda^{-1} \sinh \lambda z=z+\sum_{j=1}^{\infty} \frac{\lambda^{2 j} z^{2 j+1}}{(2 j+1) !} \\
& 4 e^{-2 \lambda t} \sinh ^{2} \lambda(z \pm t)=e^{ \pm 2 \lambda z}\left(1-e^{-2 \lambda t} e^{\mp 2 \lambda z}\right)^{2}, \\
& \sinh ^{-2} \lambda z-\cosh ^{-2} \lambda z=4 \sinh ^{-2} 2 \lambda z .
\end{aligned}
$$

The elliptic functions $\wp$ and $\zeta$ of Weierstrass type are defined by

$$
\begin{aligned}
& \wp(z)=\wp\left(z ; 2 \omega_{1}, 2 \omega_{2}\right)=\frac{1}{z^{2}}+\sum_{\omega \neq 0}\left(\frac{1}{(z-\omega)^{2}}-\frac{1}{\omega^{2}}\right), \\
& \zeta(z)=\zeta\left(z ; 2 \omega_{1}, 2 \omega_{2}\right)=\frac{1}{z}+\sum_{\omega \neq 0}\left(\frac{1}{z-\omega}+\frac{1}{\omega}+\frac{z}{\omega^{2}}\right),
\end{aligned}
$$

where the sum ranges over all non-zero periods $2 m_{1} \omega_{1}+2 m_{2} \omega_{2}\left(m_{1}, m_{2} \in \mathbb{Z}\right)$ of $\wp$. The following are some elementary properties of these functions (cf. [41]).

$$
\begin{aligned}
& \wp(z)=\wp\left(z+2 \omega_{1}\right)=\wp\left(z+2 \omega_{2}\right), \\
& \zeta^{\prime}(z)=-\wp(z), \\
& \left(\wp^{\prime}\right)^{2}=4 \wp^{3}-g_{2} \wp-g_{3}=4\left(\wp-e_{1}\right)\left(\wp-e_{2}\right)\left(\wp-e_{3}\right), \\
& e_{\nu}=\wp\left(\omega_{\nu}\right) \quad \text { for } \quad \nu=1,2,3, \quad \omega_{3}=-\omega_{1}-\omega_{2} \quad \text { and } \quad \omega_{0}=0,
\end{aligned}
$$




$$
\begin{aligned}
& \wp(2 z)=\frac{1}{4} \sum_{\nu=0}^{4} \wp\left(z+\omega_{\nu}\right)=\frac{\left(12 \wp(z)^{2}-g_{2}\right)^{2}}{16 \wp^{\prime}(z)^{2}}-2 \wp(z), \\
& \wp\left(z ; 2 \omega_{2}, 2 \omega_{1}\right)=\wp\left(z ; 2 \omega_{1}, 2 \omega_{2}\right), \\
& \wp\left(z+\omega_{1} ; 2 \omega_{1}, 2 \omega_{2}\right)=e_{1}+\frac{\left(e_{1}-e_{2}\right)\left(e_{1}-e_{3}\right)}{\wp\left(z ; 2 \omega_{1}, 2 \omega_{2}\right)-e_{1}}, \\
& \wp\left(z ; \sqrt{-1} \lambda^{-1} \pi, \infty\right)=\lambda^{2} \sinh ^{-2} \lambda z+\frac{1}{3} \lambda^{2}, \\
& \wp(z ; \infty, \infty)=z^{-2}, \\
& \wp\left(z ; \omega_{1}, 2 \omega_{2}\right)=\wp\left(z ; 2 \omega_{1}, 2 \omega_{2}\right)+\wp\left(z+\omega_{1} ; 2 \omega_{1}, 2 \omega_{2}\right)-e_{1}, \\
& \left|\begin{array}{lll}
\wp\left(z_{1}\right) & \wp^{\prime}\left(z_{1}\right) & 1 \\
\wp\left(z_{2}\right) & \wp^{\prime}\left(z_{2}\right) & 1 \\
\wp\left(z_{3}\right) & \wp^{\prime}\left(z_{3}\right) & 1
\end{array}\right|=0 \quad \text { if } \quad z_{1}+z_{2}+z_{3}=0 \\
& \wp\left(z ; 2 \omega_{1}, 2 \omega_{2}\right)=-\frac{\eta_{1}}{\omega_{1}}+\lambda^{2} \sinh ^{-2} \lambda z+\sum_{n=1}^{\infty} \frac{8 n \lambda^{2} e^{-4 n \lambda \omega_{2}}}{1-e^{-4 n \lambda \omega_{2}}} \cosh 2 n \lambda z, \\
& \eta_{1}=\zeta\left(\omega_{1} ; 2 \omega_{1}, 2 \omega_{2}\right)=\frac{\pi^{2}}{\omega_{1}}\left(\frac{1}{12}-2 \sum_{n=1}^{\infty} \frac{n e^{-4 n \lambda \omega_{2}}}{1-e^{-4 n \lambda \omega_{2}}}\right), \\
& \tau=\frac{\omega_{2}}{\omega_{1}}, \quad q=e^{\pi i \tau}=e^{-2 \lambda \omega_{2}} \quad \text { and } \quad \lambda=\frac{\pi}{2 \sqrt{-1} \omega_{1}} .
\end{aligned}
$$

Here the sums in (2.10) converge if

$$
2 \operatorname{Im} \frac{\omega_{2}}{\omega_{1}}>\frac{|z|}{\left|\omega_{1}\right|}
$$

Let $0 \leq k<2 m$. Then (2.10) means

$$
\begin{aligned}
& \wp\left(z+\frac{k}{m} \omega_{2} ; 2 \omega_{1}, 2 \omega_{2}\right)=-\frac{\eta_{1}}{\omega_{1}}+4 \lambda^{2}\left(\frac{q^{k / m} e^{-2 \lambda z}}{\left(1-e^{-2 \lambda z} q^{k / m}\right)^{2}}+\sum_{n=1}^{\infty} \frac{q^{n(2-k / m)} e^{2 n \lambda z}}{1-q^{2 n}}\right), \\
& -\frac{\eta_{1}}{\omega_{1}}=4 \lambda^{2}\left(\frac{1}{12}-2 \sum_{n=1}^{\infty} \frac{n q^{2 n}}{1-q^{2 n}}\right) .
\end{aligned}
$$

Lemma 2. Let $k$ and $m$ be integers satisfying $0<k<2 m$. Put

$$
\begin{aligned}
& \wp_{0}\left(z ; 2 \omega_{1}, 2 \omega_{2}\right)=\wp\left(z ; 2 \omega_{1}, 2 \omega_{2}\right)+\frac{\eta_{1}}{\omega_{1}}, \\
& \lambda=\frac{\pi}{2 \sqrt{-1} \omega_{1}} \quad \text { and } \quad t=q^{1 / m}=e^{\pi i \omega_{2} /\left(m \omega_{1}\right)} .
\end{aligned}
$$

Then for any bounded open set $U$ in $\mathbb{C} \times \mathbb{C}$, there exists a neighborhood $V$ of the origin of $\mathbb{C}$ such that the following statements hold.

i) $\wp_{0}\left(z ; 2 \omega_{1}, 2 \omega_{2}\right)-\lambda^{2} \sinh ^{-2} \lambda z$ and $\wp_{0}\left(z+\omega_{1} ; 2 \omega_{1}, 2 \omega_{2}\right)+\lambda^{2} \cosh ^{-2} \lambda z$ are holomorphic functions of $(z, \lambda, q) \in U \times V$ and vanish when $q=0$.

ii) $\wp_{0}\left(z+(k / m) \omega_{2} ; 2 \omega_{1}, 2 \omega_{2}\right)$ is holomorphic for $(z, \lambda, t) \in U \times V$ and has zeros of order $\min \{k, 2 m-k\}$ along the hyperplane defined by $t=0$ and satisfies

$$
\begin{array}{ll}
\left.t^{-k} \wp_{0}\left(z+\frac{k}{m} \omega_{2} ; 2 \omega_{1}, 2 \omega_{2}\right)\right|_{t=0}=4 \lambda^{2} e^{-2 \lambda z} & (0<k<m), \\
\left.t^{-k} \wp_{0}\left(z+\frac{k}{m} \omega_{2} ; 2 \omega_{1}, 2 \omega_{2}\right)\right|_{t=0}=8 \lambda^{2} \cosh 2 \lambda z & (k=m),
\end{array}
$$




$$
\left.t^{k-2 m} \wp_{0}\left(z+\frac{k}{m} \omega_{2} ; 2 \omega_{1}, 2 \omega_{2}\right)\right|_{t=0}=4 \lambda^{2} e^{2 \lambda z} \quad(m<k<2 m) .
$$

For our later convenience we list up some limiting formula discussed above. Fix $\omega_{1}$ with $\sqrt{-1} \omega_{1}>0$ and let $\omega_{2} \in \mathbb{R}$ with $\omega_{2}>0$. Then $\lambda=\pi /\left(2 \sqrt{-1} \omega_{1}\right)>0$ and

$$
\begin{aligned}
& \sinh ^{2} \lambda\left(z+\omega_{1}\right)=-\cosh ^{2} \lambda z, \quad \cosh 2 \lambda\left(z+\omega_{1}\right)=-\cosh 2 \lambda z, \\
& \lim _{\lambda \rightarrow 0} \lambda^{2} \sinh ^{-2} \lambda z=\frac{1}{z^{2}} \\
& \lim _{N \rightarrow \pm \infty} e^{2 \lambda|N|} \sinh ^{-2} \lambda(z+N)=4 e^{\mp 2 \lambda z} \text {, } \\
& \lim _{\omega_{2} \rightarrow+\infty} \wp_{0}\left(z ; 2 \omega_{1}, 2 \omega_{2}\right)=\lambda^{2} \sinh ^{-2} \lambda z, \\
& \lim _{\omega_{2} \rightarrow+\infty} \wp_{0}\left(z+\omega_{1} ; 2 \omega_{1}, 2 \omega_{2}\right)=-\lambda^{2} \cosh ^{-2} \lambda z \\
& \lim _{\omega_{2} \rightarrow \infty} e^{2 r \lambda \omega_{2}} \wp_{0}\left(z+r \omega_{2} ; 2 \omega_{1}, 2 \omega_{2}\right)=4 \lambda^{2} e^{-2 \lambda z} \quad \text { if } \quad 0<r<1 \text {, } \\
& \lim _{\omega_{2} \rightarrow \infty} e^{2 \lambda \omega_{2}} \wp_{0}\left(z+\omega_{2} ; 2 \omega_{1}, 2 \omega_{2}\right)=8 \lambda^{2} \cosh 2 \lambda z, \\
& \lim _{\omega_{2} \rightarrow \infty} e^{2(2-r) \lambda \omega_{2}} \wp_{0}\left(z+r \omega_{2} ; 2 \omega_{1}, 2 \omega_{2}\right)=4 \lambda^{2} e^{2 \lambda z} \quad \text { if } \quad 1<r<2 .
\end{aligned}
$$

\section{Type $A_{n-1}(n \geq 3)$}

The completely integrable Schrödinger operator of type $A_{n-1}$ is of the form

$$
P=-\frac{1}{2} \sum_{j=1}^{n} \frac{\partial^{2}}{\partial x_{j}^{2}}+\sum_{1 \leq i<j \leq n} u_{i j}^{-}\left(x_{i}-x_{j}\right) .
$$

Denoting

$$
u_{e_{i}-e_{j}}(x)=u_{e_{j}-e_{i}}(x)=u_{i j}^{-}\left(x_{i}-x_{j}\right),
$$

we put

$$
\begin{aligned}
P_{k} & =\sum_{0 \leq \nu \leq k / 2} \frac{1}{2^{\nu} \nu !(k-2 \nu) !(n-k) !} \sum_{w \in \mathfrak{S}_{n}} u_{w\left(e_{1}-e_{2}\right)} u_{w\left(e_{3}-e_{4}\right)} \cdots u_{w\left(e_{2 \nu-1}-e_{2 \nu}\right)} \partial_{w\left(e_{2 \nu+1}\right)} \cdots \partial_{w\left(e_{k}\right)} \\
& =\sum_{0 \leq \nu \leq k / 2} \sum u_{i_{1} i_{2}}^{-} \cdots u_{i_{2 \nu-1} i_{2 \nu}}^{-} \partial_{i_{2 \nu+1}} \cdots \partial_{i_{k}}
\end{aligned}
$$

according to the integrals given in $[25,32]$. We will examine the functions $u_{i j}^{-}(t)$ which satisfy

$$
\left[P_{i}, P_{j}\right]=0 \quad \text { for } \quad 1 \leq i<j \leq n
$$

Here we note that

$$
\begin{aligned}
& P=P_{2}-\frac{1}{2} P_{1}^{2}, \quad P_{1}=\partial_{1}+\cdots+\partial_{n}, \\
& P_{2}=\sum_{1 \leq i<j \leq n} \partial_{i} \partial_{j}+\sum_{1 \leq i<j \leq n} u_{i j}^{-}\left(x_{i}-x_{j}\right), \\
& P_{3}=\sum_{1 \leq i<j<k \leq n} \partial_{i} \partial_{j} \partial_{k}+\sum_{k=1}^{n} \sum_{\substack{1 \leq i<j \leq n \\
i \neq k, j \neq k}} u_{i j}^{-}\left(x_{i}-x_{j}\right) \partial_{k} .
\end{aligned}
$$




$$
\begin{aligned}
P_{4} & =\sum_{1 \leq i<j<k<\ell \leq n} \partial_{i} \partial_{j} \partial_{k} \partial_{\ell}+\sum_{1 \leq k<\ell \leq n} \sum_{\substack{1 \leq i<j \leq n \\
i \neq k, \ell, j \neq k, \ell}} u_{i j}^{-} \partial_{k} \partial_{\ell}+\sum_{1 \leq i<j<k<\ell \leq n}\left(u_{i j}^{-} u_{k \ell}^{-}+u_{i k}^{-} u_{j \ell}^{-}+u_{i \ell}^{-} u_{j k}^{-}\right) \\
& =\sum \partial_{i} \partial_{j} \partial_{k} \partial_{\ell}+\sum u_{i j}^{-} \partial_{k} \partial_{\ell}+\sum u_{i j}^{-} u_{k \ell}^{-}, \\
P_{5} & =\sum \partial_{i} \partial_{j} \partial_{k} \partial_{\ell} \partial_{m}+\sum u_{i j}^{-} \partial_{k} \partial_{\ell} \partial_{m}+\sum u_{i j}^{-} u_{k \ell}^{-} \partial_{m}, \\
P_{6} & =\sum \partial_{i} \partial_{j} \partial_{k} \partial_{\ell} \partial_{m} \partial_{\nu}+\sum u_{i j}^{-} \partial_{k} \partial_{\ell} \partial_{m} \partial_{\nu}+\sum u_{i j}^{-} u_{k \ell}^{-} \partial_{m} \partial_{\nu}+\sum u_{i j}^{-} u_{k \ell}^{-} u_{m \nu}^{-},
\end{aligned}
$$

Since $W\left(A_{n-1}\right)$ is naturally isomorphic to the permutation group $\mathfrak{S}_{n}$ of the set $\{1, \ldots, n\}$, we will identify them. In $[25,32]$, the integrable potentials of type $A_{n-1}$ which are invariant under the action of $\mathfrak{S}_{n}$ are determined and moreover (3.2) with (3.1) is proved. They are

$$
u_{e_{i}-e_{j}}(x)=u\left(\left\langle e_{i}-e_{j}, x\right\rangle\right)
$$

with an even function $u$ and

(Ellip- $\left.A_{n-1}\right)$ Elliptic potential of type $A_{n-1}$ :

$$
\begin{aligned}
& u(t)=C \wp_{0}\left(t ; 2 \omega_{1}, 2 \omega_{2}\right), \\
& R_{E}\left(A_{n-1} ; x_{1}, \ldots, x_{n} ; C, 2 \omega_{1}, 2 \omega_{2}\right)=C \sum_{1 \leq i<j \leq n} \wp_{0}\left(x_{i}-x_{j} ; 2 \omega_{1}, 2 \omega_{2}\right),
\end{aligned}
$$

(Trig- $\left.A_{n-1}\right)$ Trigonometric potential of type $A_{n-1}$ :

$$
u(t)=C \sinh ^{-2} \lambda t, \quad R_{T}\left(A_{n-1} ; x_{1}, \ldots, x_{n} ; C, \lambda\right)=C \sum_{1 \leq i<j \leq n} \sinh ^{-2} \lambda\left(x_{i}-x_{j}\right),
$$

(Rat- $\left.A_{n-1}\right)$ Rational potential of type $A_{n-1}$ :

$$
u(t)=\frac{C}{t^{2}}, \quad R_{R}\left(A_{n-1} ; x_{1}, \ldots, x_{n} ; C\right)=\sum_{1 \leq i<j \leq n} \frac{C}{\left(x_{i}-x_{j}\right)^{2}} .
$$

We review how the integrability of (Ellip- $\left.A_{n-1}\right)$ implies the integrability of other systems.

Since it follows from (2.14) that

$$
\begin{aligned}
& \lim _{\omega_{2} \rightarrow \infty} R_{E}\left(A_{n-1} ; x ; \frac{C}{\lambda^{2}}, 2 \omega_{1}, 2 \omega_{2}\right)=R_{T}\left(A_{n-1} ; x ; C, \lambda\right), \\
& u(t)=\lim _{\omega_{2} \rightarrow \infty} \frac{C}{\lambda^{2}} \wp_{0}\left(t ; 2 \omega_{1}, 2 \omega_{2}\right)=C \sinh ^{-2} \lambda t,
\end{aligned}
$$

the integrability (3.2) for (Trig- $A_{n-1}$ ) follows from that for (Ellip- $A_{n-1}$ ) by the analytic continuation of $u_{e_{i}-e_{j}}(x)$ and $P_{k}$ with respect to $q$ (cf. (2.10), (3.1), (3.3) and Lemma 2 i)).

The integrability for (Rat- $\left.A_{n-1}\right)$ is similarly follows in view of Lemma 1 with

$$
\begin{aligned}
& \lim _{\lambda \rightarrow 0} R_{T}\left(A_{n-1} ; x ; \lambda^{2} C, \lambda\right)=R_{R}\left(A_{n-1} ; x ; C\right), \\
& u(t)=\lim _{\lambda \rightarrow 0} \lambda^{2} C \sinh ^{-2} \lambda t .
\end{aligned}
$$

This argument using the analytic continuation for the proof of (3.2) is given in [32].

As is shown [33], there are two other integrable potentials of type $A_{n-1}$ to which this argument can be applied. 
(Toda- $A_{n-1}^{(1)}$ ) Toda potential of type $A_{n-1}^{(1)}$ :

$$
R_{L}\left(A_{n-1}^{(1)} ; x ; C, \lambda\right)=\sum_{i=1}^{n-1} C e^{\lambda\left(x_{i}-x_{i+1}\right)}+C e^{\lambda\left(x_{n}-x_{1}\right)},
$$

(Toda- $A_{n-1}$ ) Toda potential of type $A_{n-1}$ :

$$
R_{L}\left(A_{n-1} ; x ; C, \lambda\right)=\sum_{i=1}^{n-1} C e^{\lambda\left(x_{i}-x_{i+1}\right)} .
$$

The integrability (3.2) for these potentials similarly follows in view of Lemmas 1, 2 and

$$
\begin{aligned}
\lim _{\omega_{2} \rightarrow \infty} R_{E}\left(A_{n-1} ; x_{1}-\frac{2 \omega_{2}}{n}, \ldots, x_{k}-\frac{2 k \omega_{2}}{n}, \ldots, x_{n}-2 \omega_{2} ; \frac{e^{(4 / n) \lambda \omega_{2}}}{4 \lambda^{2}} C, 2 \omega_{1}, 2 \omega_{2}\right) \\
=R_{L}\left(A_{n-1}^{(1)} ; x ; C,-2 \lambda\right), \\
u_{e_{i}-e_{j}}(x)=\lim _{\omega_{2} \rightarrow \infty} \frac{e^{(4 / n) \lambda \omega_{2}}}{4 \lambda^{2}} C \wp_{0}\left(x_{i}-x_{j}+\frac{2(j-i) \omega_{2}}{n} ; 2 \omega_{1}, 2 \omega_{2}\right) \\
\quad=\left\{\begin{array}{lll}
C e^{-2 \lambda\left(x_{i}-x_{i+1}\right)} & \text { if } 1<j=i+1 \leq n, \\
C e^{-2 \lambda\left(x_{n}-x_{1}\right)} & \text { if } i=1 \text { and } j=n, \\
0 & \text { if } 1 \leq i<j \leq n \text { and } j-i \neq 1, n-1
\end{array}\right.
\end{aligned}
$$

and

$$
\begin{aligned}
\lim _{N \rightarrow \infty} R_{T} & \left(A_{n-1} ; x_{1}-N, \ldots, x_{n}-n N ; \frac{e^{2 \lambda N}}{4} C, \lambda\right)=R_{L}\left(A_{n-1} ; x ; C,-2 \lambda\right), \\
u_{e_{i}-e_{j}}(x) & =\lim _{N \rightarrow \infty} \frac{e^{2 \lambda N}}{4} C \sinh ^{-2} \lambda\left(x_{i}-x_{j}+(j-i) N\right) \\
& =\left\{\begin{array}{lll}
C e^{-2 \lambda\left(x_{i}-x_{i+1}\right)} & \text { if } & 1<j=i+1 \leq n, \\
0 & \text { if } 1 \leq i<j \leq n \text { and } j \neq i+1,
\end{array}\right.
\end{aligned}
$$

respectively, if $\operatorname{Re} \lambda>0$. The restriction $\operatorname{Re} \lambda>0$ is removed also by the analytic continuation.

Thus the following theorem is obtained by the analytic continuation of the integrals (3.1) of (Ellip- $A_{n-1}$ ) whose commutativity (3.2) is assured by [32].

Theorem $1\left(A_{n-1},[10,11,28,32,33,34]\right.$, etc.). The Schrödinger operators with the potential functions (Ellip- $\left.A_{n-1}\right),\left(\right.$ Trig- $\left.A_{n-1}\right),\left(\right.$ Rat- $\left.A_{n-1}\right),\left(\right.$ Toda- $\left.A_{n-1}^{(1)}\right)$ and (Toda- $\left.A_{n-1}\right)$ are completely integrable and their integrals are given by (3.1) with $u_{e_{i}-e_{j}}(x)$ in the above.

Remark 1. i) There are quite many papers studying these Schrödinger operators of type $A_{n-1}$. The proof of this theorem using analytic continuation is explained in [33].

ii) The complete integrability (3.2) for (Ellip- $\left.A_{n-1}\right)$ is first established by [32, Theorem 5.2], whose proof is as follows. The equations $\left[P_{1}, P_{k}\right]=\left[P_{2}, P_{k}\right]=0$ for $k=1, \ldots, n$ are easily obtained by direct calculations with the formula (2.9) (cf. [32, Lemma 5.1]). Then the relation $\left[P_{2},\left[P_{i}, P_{j}\right]\right]=0$ and periodicity and symmetry of $P_{k}$ imply $\left[P_{i}, P_{j}\right]=0$ (cf. [32, Lemma 3.5]). Note that the proof of the integrability given in [28, $\S 5$ Proposition 2 and Appendix E] is not correct as is clarified in [32, Remark 3.7] (cf. [33, § 4.2]).

Note that the complete integrability for (Trig- $A_{n-1}$ ) is shown in [34].

iii) If $\operatorname{Re} \lambda>0$, we also have

$$
\lim _{N \rightarrow \infty} R_{L}\left(A_{n-1}^{(1)} ; x_{k}+k N ; C e^{-2 \lambda N} ;-2 \lambda\right)=R_{L}\left(A_{n-1} ; x ; C,-2 \lambda\right) .
$$


iv) Note that

$$
R_{L}\left(A_{n-1} ; x_{k}+N_{k} ; C, \lambda\right)=\sum_{i=1}^{n-1} C e^{\lambda\left(N_{i}-N_{i+1}\right)} \cdot e^{\lambda\left(x_{i}-x_{i+1}\right)} .
$$

Hence $e^{\lambda\left(x_{1}-x_{2}\right)}-e^{\lambda\left(x_{2}-x_{3}\right)}$ gives the potential function of a completely integrable system of type $A_{n-1}$ with $n=3$ but the potential function

$$
\lim _{\lambda \rightarrow 0} \lambda^{-1}\left(e^{\lambda\left(x_{1}-x_{2}\right)}-e^{\lambda\left(x_{2}-x_{3}\right)}\right)=x_{1}-2 x_{2}+x_{3}
$$

does not give such a system because it does not satisfy (9.3) in Remark 13.

Considering the limit of the parameters of the integrable potential function, we should take care of the limit of integrals.

v) Let $P_{n}(t)$ denote the differential operator $P_{n}$ in (3.1) defined by replacing $u_{i j}^{-}$by $\tilde{u}_{i j}^{-}=u_{i j}^{-}+t$ with a constant $t \in \mathbb{C}$. Then

$$
\begin{aligned}
& P_{n}(t)=\sum_{0 \leq k \leq n / 2} \frac{(2 k) !}{2^{k} k !} P_{n-2 k} t^{k} \quad \text { with } \quad P_{0}=1, \\
& {\left[P_{n}(s), P_{n}(t)\right]=0 \quad \text { for } \quad s, t \in \mathbb{C} .}
\end{aligned}
$$

In fact, the term $u_{12}^{-} u_{34}^{-} \cdots u_{2 j-1,2 j}^{-} \partial_{2 j+1} \partial_{2 j+2} \cdots \partial_{n-2 k}$ appears only in the coefficient of $t^{k}$ in the right hand side of (3.4) and it is contained in the terms

$$
\tilde{u}_{i_{n-2 k+1} i_{n-2 k+2}} \cdots \tilde{u}_{i_{n-1} i_{n}}^{-} \tilde{u}_{12}^{-} \cdots \tilde{u}_{2 j-1,2 j}^{-} \partial_{2 j+1} \cdots \partial_{n-2 k}
$$

of $P_{n}(t)$, where the number of the possibilities of these $\tilde{u}_{i_{n-2 k+1} i_{n-2 k+2}} \cdots \tilde{u}_{i_{n-1} i_{n}}$ is $(2 k) ! /\left(2^{k} k !\right)$ because $\left\{i_{n-2 k+1}, i_{n-2 k+2}, \ldots, i_{n}\right\}=\{n-2 k+1, \ldots, n\}$.

vi) Since

$$
P_{k-1}=(n-k+1)\left[P_{k}, x_{1}+\cdots+x_{n}\right] \quad \text { for } \quad k=2, \ldots n,
$$

$\left[P_{k}, P_{2}\right]=0$ implies $\left[P_{k-1}, P_{2}\right]=0$ by the Jacobi identity. Here we note that

$$
\left[u_{12}^{-} u_{34}^{-} \cdots u_{2 j-1,2 j}^{-} \partial_{2 j+1} \cdots \partial_{k-1} \partial_{\nu}, x_{\nu}\right]=u_{12}^{-} u_{34}^{-} \cdots u_{2 j-1,2 j}^{-} \partial_{2 j+1} \cdots \partial_{k-1}
$$

for $\nu=k, k+1, \ldots, n$.

vi) The potential functions of (Trig- $\left.A_{n-1}\right)$, (Rat- $\left.A_{n-1}\right)$ and (Toda- $\left.A_{n-1}\right)$ are specializations of more general integrable potential functions of type $B_{n}$ (cf. Definition 5).

In the following diagram we show the relations among integrable potentials of type $A_{n-1}$ by taking limits.

Hierarchy of Integrable Potentials of Type $A_{n-1}(n \geq 3)$

$$
\begin{aligned}
& \text { Toda- } A_{n-1}^{(1)} \rightarrow \text { Toda- } A_{n-1} \\
& \text { Ellip- } A_{n-1} \quad \rightarrow \quad \text { Trig- } A_{n-1} \quad \rightarrow \quad \text { Rat- } A_{n-1}
\end{aligned}
$$




\section{Type $B_{2}$}

In this section we study the following commuting differential operators $P$ and $P_{2}$.

$$
\begin{aligned}
& P=-\frac{1}{2}\left(\frac{\partial^{2}}{\partial x^{2}}+\frac{\partial^{2}}{\partial y^{2}}\right)+R(x, y), \\
& P_{2}=\frac{\partial^{4}}{\partial x^{2} \partial y^{2}}+S \quad \text { with } \quad \text { ord } S<4, \\
& {\left[P, P_{2}\right]=0 .}
\end{aligned}
$$

The Schrödinger operators $P$ of type $B_{2}$ in this section are known to be completely integrable. They are listed in $[23,24,30]$. We review them and give the explicit expression of $P_{2}$.

First we review the arguments given in [23, 24]. Since $P$ is self-adjoint, we may assume $P_{2}$ is also self-adjoint by replacing $P_{2}$ by its self-adjoint part if necessary. Here for

$$
A=\sum a_{i j}(x, y) \frac{\partial^{i+j}}{\partial x^{i} \partial y^{j}}
$$

we define

$$
{ }^{t} A=\sum(-1)^{i+j} \frac{\partial^{i+j}}{\partial x^{i} \partial y^{j}} a_{i j}(x, y)
$$

and $A$ is called self-adjoint if ${ }^{t} A=A$.

Lemma 3 ([23]). Suppose $P$ and $P_{2}$ are self-adjoint operators satisfying (4.1). Then

$$
\begin{aligned}
& R(x, y)=u^{+}(x+y)+u^{-}(x-y)+v(x)+w(y), \\
& P_{2}=\left(\frac{\partial^{2}}{\partial x \partial y}+u^{-}(x-y)-u^{+}(x+y)\right)^{2}-2 w(y) \frac{\partial^{2}}{\partial x^{2}}-2 v(x) \frac{\partial^{2}}{\partial y^{2}}+4 v(x) w(y)+T(x, y)
\end{aligned}
$$

and the function $T(x, y)$ satisfies

$$
\begin{aligned}
& \frac{\partial T(x, y)}{\partial x}=2\left(u^{+}(x+y)-u^{-}(x-y)\right) \frac{\partial w(y)}{\partial y}+4 w(y) \frac{\partial}{\partial y}\left(u^{+}(x+y)-u^{-}(x-y)\right), \\
& \frac{\partial T(x, y)}{\partial y}=2\left(u^{+}(x+y)-u^{-}(x-y)\right) \frac{\partial v(x)}{\partial x}+4 v(x) \frac{\partial}{\partial x}\left(u^{+}(x+y)-u^{-}(x-y)\right) .
\end{aligned}
$$

Conversely, if a function $T(x, y)$ satisfies (4.3) for suitable functions $u^{ \pm}(t), v(t)$ and $w(t)$, then (4.1) is valid for $R(x, y)$ and $P_{2}$ defined by (4.2).

Remark 2. i) If $w(y)=0$, then $T(x, y)$ does not depend on $x$.

ii) The self-adjointness of $P_{2}$ and the vanishing of the third order term of $\left[P, P_{2}\right]$ imply that $P_{2}$ should be of the form (4.2) with a suitable function $T(x, y)$. Then the vanishing of the first order term implies (4.3). The last claim in Lemma 3 is obtained by direct calculation.

Since $T(x, y)$ satisfying (4.3) is determined by $\left(u^{-}, u^{+} ; v, w\right)$ up to the difference of constants, we will write $T\left(u^{-}, u^{+} ; v, w\right)$ for the corresponding $T(x, y)$ which is an element of the space of meromorphic functions of $(x, y)$ modulo constant functions and define $Q\left(u^{-}, u^{+} ; v, w\right)$ by

$$
T\left(u^{-}, u^{+} ; v, w\right)=2\left(u^{-}(x-y)+u^{+}(x+y)\right)(v(x)+w(y))-4 Q\left(u^{-}, u^{+} ; v, w\right) .
$$

Note that $T\left(u^{-}, u^{+} ; v, w\right)$ and $Q\left(u^{-}, u^{+} ; v, w\right)$ are defined only if the function $T(x, y)$ satisfying (4.3) exists. The following lemma is a direct consequence of (4.3) and this definition of $Q$. 
Lemma 4 ([23]). Suppose $T\left(u^{-}, u^{+} ; v, w\right)$ and $T\left(u_{i}^{-}, u_{i}^{+} ; v_{j}, w_{j}\right)$ are defined. Then for any $C$, $C_{i}, C_{j}^{\prime} \in \mathbb{C}$ the left hand sides of the following identities are also defined and

$$
\begin{aligned}
& \left.T\left(u^{-}(t)+C, u^{+}(t)+C ; v(t), w(t)\right)\right)=T\left(u^{-}(t), u^{+}(t) ; v(t), w(t)\right), \\
& \left.Q\left(u^{-}(t), u^{+}(t) ; v(t)+C, w(t)+C\right)\right)=Q\left(u^{-}(t), u^{+}(t) ; v(t), w(t)\right), \\
& Q\left(u^{-}(C t), u^{+}(C t) ; v(C t), w(C t)\right)=\left.Q\left(u^{-}(t), u^{+}(t) ; v(t), w(t)\right)\right|_{x \rightarrow C x, y \rightarrow C y,} \\
& Q\left(\sum_{i=1}^{2} A_{i} u_{i}^{-}, \sum_{i=1}^{2} A_{i} u_{i}^{+} ; \sum_{j=1}^{2} C_{j} v_{j}, \sum_{j=1}^{2} C_{j} w_{j}\right)=\sum_{i=1}^{2} \sum_{j=1}^{2} A_{i} C_{j} Q\left(u_{i}^{-}, u_{i}^{+} ; v_{j}, w_{j}\right) .
\end{aligned}
$$

Hence the left hand sides give pairs $P$ and $P_{2}$ with $\left[P, P_{2}\right]=0$.

For simplicity we will use the notation

$$
Q\left(u^{-}, u^{+} ; v\right)=Q\left(u^{-}, u^{+} ; v, v\right), \quad Q(u ; v, w)=Q(u, u ; v, w), \quad Q(u ; v)=Q(u, u ; v, v) .
$$

The same convention will be also used for $T\left(u^{-}, u^{+} ; v, w\right)$. The integrable potentials of type $B_{2}$ in this note are classified into three kinds. The potentials of the first kind are the unified integrable potentials which are in the same form as those of type $B_{n}$ with $n \geq 3$, which we call normal integrable potentials of type $B_{2}$.

The integrable potentials of type $B_{2}$ admit a special transformation called dual which does not exist in $B_{n}$ with $n \geq 3$. Hence there are normal potentials and their dual in the invariant integrable potentials of type $B_{2}$. Because of this duality, there exist another kind of invariant integrable potentials of type $B_{2}$, which we call special integrable potentials of type $B_{2}$.

In this section we present $(R(x, y), T(x, y))$ as suitable limits of elliptic functions as in the previous section since it helps to study the potentials of type $B_{n}$ in Section 5 . We reduce the complete integrability of the limits to that of a systems with elliptic potentials. But we can also check (4.3) by direct calculations (cf. Remark 5).

\subsection{Normal case}

In this subsection we study the integrable systems (4.1) with (4.2) which have natural extension to type $B_{n}$ for $n \geq 3$ and have the form

$$
u^{-}(t)=A u_{0}^{-}(t), \quad u^{+}(t)=A u_{0}^{+}(t), \quad v(t)=\sum_{j=0}^{3} C_{j} v_{j}(t), \quad w(t)=\sum_{j=0}^{3} C_{j} w_{j}(t)
$$

with any $A, C_{0}, C_{1}, C_{2}, C_{3} \in \mathbb{C}$. These systems are expressed by the symbol

$$
\left(\left\langle u_{0}^{-}\right\rangle,\left\langle u_{0}^{+}\right\rangle ;\left\langle v_{0}, v_{1}, v_{2}, v_{3}\right\rangle,\left\langle w_{0}, w_{1}, w_{2}, w_{3}\right\rangle\right) .
$$

The most general system is the following (Ellip- $\left.B_{2}\right)$ defined by elliptic functions, which is called Inozemtsev model [12].

Theorem 2 ( $B_{2}$ : Normal case, $[12,24,23,30]$ etc.). The operators $P$ and $P_{2}$ defined by the following pairs of $R(x, y)$ and $T(x, y)$ satisfy (4.1) and (4.2).

(Ellip- $\left.B_{2}\right): \quad\left(\langle\wp(t)\rangle ;\left\langle\wp(t), \wp\left(t+\omega_{1}\right), \wp\left(t+\omega_{2}\right), \wp\left(t+\omega_{3}\right)\right\rangle\right)$

$$
v(x)=\sum_{j=0}^{3} C_{j} \wp\left(x+\omega_{j}\right), \quad w(y)=\sum_{j=0}^{3} C_{j} \wp\left(y+\omega_{j}\right),
$$




$$
\begin{aligned}
& u^{-}(x-y)=A \wp(x-y), \quad u^{+}(x+y)=A \wp(x+y), \\
& R(x, y)=A(\wp(x-y)+\wp(x+y))+\sum_{j=0}^{3} C_{j}\left(\wp\left(x+\omega_{j}\right)+\wp\left(y+\omega_{j}\right)\right), \\
& T(x, y)=2 A(\wp(x-y)+\wp(x+y))\left(\sum_{j=0}^{3} C_{j}\left(\wp\left(x+\omega_{j}\right)+\wp\left(y+\omega_{j}\right)\right)\right. \\
& -4 A \sum_{j=0}^{3} C_{j} \wp\left(x+\omega_{j}\right) \wp\left(y+\omega_{j}\right) .
\end{aligned}
$$

$\left(\right.$ Trig- $\left.B_{2}\right): \quad\left(\left\langle\sinh ^{-2} \lambda t\right\rangle ;\left\langle\sinh ^{-2} \lambda t, \sinh ^{-2} 2 \lambda t, \sinh ^{2} \lambda t, \sinh ^{2} 2 \lambda t\right\rangle\right)$

$$
\begin{aligned}
& v(x)=C_{0} \sinh ^{-2} \lambda x+C_{1} \cosh ^{-2} \lambda x+C_{2} \sinh ^{2} \lambda x+\frac{1}{4} C_{3} \sinh ^{2} 2 \lambda x, \\
& w(y)=C_{0} \sinh ^{-2} \lambda y+C_{1} \cosh ^{-2} \lambda y+C_{2} \sinh ^{2} \lambda y+\frac{1}{4} C_{3} \sinh ^{2} 2 \lambda y, \\
& u^{-}(x-y)=A \sinh ^{-2} \lambda(x-y), \quad u^{+}(x+y)=A \sinh ^{-2} \lambda(x+y), \\
& R(x, y)=A\left(\sinh ^{-2} \lambda(x-y)+\sinh ^{-2} \lambda(x+y)\right)+C_{0}\left(\sinh ^{-2} \lambda x+\sinh ^{-2} \lambda y\right) \\
&+C_{1}\left(\cosh ^{-2} \lambda x+\cosh ^{-2} \lambda y\right)+C_{2}\left(\sinh ^{2} \lambda x+\sinh ^{2} \lambda y\right) \\
&+\frac{1}{4} C_{3}\left(\sinh ^{2} 2 \lambda x+\sinh ^{2} 2 \lambda y\right), \\
& T(x, y)=2 A\left(\sinh ^{-2} \lambda(x-y)+\sinh ^{-2} \lambda(x+y)\right) \\
& \quad+\left(C_{0}\left(\sinh ^{-2} \lambda x+\sinh ^{-2} \lambda y\right)+C_{1}\left(\cosh ^{-2} \lambda x+\cosh ^{-2} \lambda y\right)\right. \\
&\left.+C_{2}\left(\sinh ^{2} \lambda x+\sinh ^{2} \lambda y\right)+\frac{1}{4} C_{3}\left(\sinh ^{2} 2 \lambda x+\sinh ^{2} 2 \lambda y\right)\right) \\
& \quad-4 A\left(C_{0} \sinh ^{-2} \lambda x \cdot \sinh ^{-2} \lambda y-C_{1} \cosh ^{-2} \lambda x \cdot \cosh ^{-2} \lambda y\right. \\
&\left.+C_{3}\left(\sinh ^{2} \lambda x+\sinh ^{2} \lambda y+2 \sinh ^{2} \lambda x \cdot \sinh ^{2} \lambda y\right)\right) .
\end{aligned}
$$

$\left(\right.$ Rat- $\left.B_{2}\right): \quad\left(\left\langle t^{-2}\right\rangle ;\left\langle t^{-2}, t^{2}, t^{4}, t^{6}\right\rangle\right)$

$$
\begin{aligned}
& v(x)=C_{0} x^{-2}+C_{1} x^{2}+C_{2} x^{4}+C_{3} x^{6}, \quad w(y)=C_{0} y^{-2}+C_{1} y^{2}+C_{2} y^{4}+C_{3} y^{6}, \\
& u^{-}(x-y)=\frac{A}{(x-y)^{2}}, \quad u^{+}(x+y)=\frac{A}{(x+y)^{2}}, \\
& R(x, y)=\frac{A}{(x-y)^{2}}+\frac{A}{(x+y)^{2}}+C_{0}\left(x^{-2}+y^{-2}\right)+C_{1}\left(x^{2}+y^{2}\right)+C_{2}\left(x^{4}+y^{4}\right)+C_{3}\left(x^{6}+y^{6}\right), \\
& T(x, y)=2\left(u^{-}(x-y)+u^{+}(x+y)\right)(v(x)+w(y))-4 A\left(\frac{C_{0}}{x^{2} y^{2}}+C_{2}\left(x^{2}+y^{2}\right)\right. \\
& \left.\quad+C_{3}\left(x^{4}+y^{4}+3 x^{2} y^{2}\right)\right)=8 A \frac{2 C_{0}+2 C_{1} x^{2} y^{2}+C_{2} x^{2} y^{2}\left(x^{2}+y^{2}\right)+2 C_{3} x^{4} y^{4}}{\left(x^{2}-y^{2}\right)^{2}} .
\end{aligned}
$$

(Toda- $D_{2}^{(1)}$-bry): $\quad\left(\langle\cosh 2 \lambda t\rangle ;\left\langle\sinh ^{-2} \lambda t, \sinh ^{-2} 2 \lambda t\right\rangle,\left\langle\sinh ^{-2} \lambda t, \sinh ^{-2} 2 \lambda t\right\rangle\right)$

$$
\begin{aligned}
& v(x)=C_{0} \sinh ^{-2} \lambda x+C_{1} \sinh ^{-2} 2 \lambda x, \quad w(y)=C_{2} \sinh ^{-2} \lambda y+C_{3} \sinh ^{-2} 2 \lambda y, \\
& u^{-}(x-y)=A \cosh 2 \lambda(x-y), \quad u^{+}(x+y)=A \cosh 2 \lambda(x+y), \\
& R(x, y)=A \cosh 2 \lambda(x-y)+A \cosh 2 \lambda(x+y) \\
& \quad+C_{0} \sinh ^{-2} \lambda x+C_{1} \sinh ^{-2} 2 \lambda x+C_{2} \sinh ^{-2} \lambda y+C_{3} \sinh ^{-2} 2 \lambda y,
\end{aligned}
$$


$T(x, y)=8 A\left(C_{0} \cosh 2 \lambda y+C_{2} \cosh 2 \lambda x\right)$.

(Toda- $B_{2}^{(1)}$-bry): $\quad\left(\left\langle e^{-2 \lambda t}\right\rangle ;\left\langle e^{2 \lambda t}, e^{4 \lambda t}\right\rangle,\left\langle\sinh ^{-2} \lambda t, \sinh ^{-2} 2 \lambda t\right\rangle\right)$

$$
\begin{aligned}
& v(x)=C_{0} e^{2 \lambda x}+C_{1} e^{4 \lambda x}, \quad w(y)=C_{2} \sinh ^{-2} \lambda y+C_{3} \sinh ^{-2} 2 \lambda y, \\
& u^{-}(x-y)=A e^{-2 \lambda(x-y)}, \quad u^{+}(x+y)=A e^{-2 \lambda(x+y)}, \\
& R(x, y)=A e^{-2 \lambda(x-y)}+A e^{-2 \lambda(x+y)}+C_{0} e^{2 \lambda x}+C_{1} e^{4 \lambda x}+C_{2} \sinh ^{-2} \lambda y+C_{3} \sinh ^{-2} 2 \lambda y, \\
& T(x, y)=4 A\left(C_{0} \cosh 2 \lambda y+2 C_{2} e^{-2 \lambda x}\right) .
\end{aligned}
$$

(Trig- $A_{1}^{(1)}$-bry): $\quad\left(\left\langle\sinh ^{-2} \lambda t\right\rangle, 0 ;\left\langle e^{-2 \lambda t}, e^{-4 \lambda t}, e^{2 \lambda t}, e^{4 \lambda t}\right\rangle\right)$

$$
\begin{aligned}
& v(x)=C_{0} e^{-2 \lambda x}+C_{1} e^{-4 \lambda x}+C_{2} e^{2 \lambda x}+C_{3} e^{4 \lambda x}, \\
& \begin{aligned}
w(y)=C_{0} e^{-2 \lambda y}+C_{1} e^{-4 \lambda y}+C_{2} e^{2 \lambda y}+C_{3} e^{4 \lambda y}, \\
u^{-}(x-y)=A \sinh ^{-2} \lambda(x-y), \quad u^{+}(x+y)=0, \\
R(x, y)=A \sinh ^{-2} \lambda(x-y)+C_{0}\left(e^{-2 \lambda x}+e^{-2 \lambda y}\right)+C_{1}\left(e^{-4 \lambda x}+e^{-4 \lambda y}\right) \\
\quad+C_{2}\left(e^{2 \lambda x}+e^{2 \lambda y}\right)+C_{3}\left(e^{4 \lambda x}+e^{4 \lambda y}\right), \\
T(x, y)=2 A \sinh ^{-2} \lambda(x-y)\left(C_{0}\left(e^{-2 \lambda x}+e^{-2 \lambda y}\right)\right. \\
\left.\quad+2 C_{1} e^{-2 \lambda(x+y)}+C_{2}\left(e^{2 \lambda x}+e^{2 \lambda y}\right)+2 C_{3} e^{2 \lambda(x+y)}\right) .
\end{aligned}
\end{aligned}
$$

$\left(\right.$ Toda- $\left.C_{2}^{(1)}\right): \quad\left(\left\langle e^{-2 \lambda t}\right\rangle, 0 ;\left\langle e^{2 \lambda t}, e^{4 \lambda t}\right\rangle,\left\langle e^{-2 \lambda t}, e^{-4 \lambda t}\right\rangle\right)$

$$
\begin{aligned}
& v(x)=C_{0} e^{2 \lambda x}+C_{1}^{4 \lambda x}, \quad w(y)=C_{2} e^{-2 \lambda y}+C_{3} e^{-4 \lambda y}, \\
& u^{-}(x-y)=A e^{-2 \lambda(x-y)}, \quad u^{+}(x+y)=0, \\
& R(x, y)=A e^{-2 \lambda(x-y)}+C_{0} e^{2 \lambda x}+C_{1}^{4 \lambda x}+C_{2} e^{-2 \lambda y}+C_{3} e^{-4 \lambda y}, \\
& T(x, y)=2 A\left(C_{0} e^{2 \lambda y}+C_{2} e^{-2 \lambda x}\right) .
\end{aligned}
$$

(Rat- $A_{1}$-bry): $\quad\left(\left\langle t^{-2}\right\rangle, 0 ;\left\langle t, t^{2}, t^{3}, t^{4}\right\rangle\right)$

$$
\begin{aligned}
& v(x)=C_{0} x+C_{1} x^{2}+C_{2} x^{3}+C_{3} x^{4}, \quad w(y)=C_{0} y+C_{1} y^{2}+C_{2} y^{3}+C_{3} y^{4}, \\
& u^{-}(x-y)=\frac{A}{(x-y)^{2}}, \quad u^{+}(x+y)=0, \\
& R(x, y)=\frac{A}{(x-y)^{2}}+C_{0}(x+y)+C_{1}\left(x^{2}+y^{2}\right)+C_{2}\left(x^{3}+y^{3}\right)+C_{3}\left(x^{4}+y^{4}\right), \\
& T(x, y)=\frac{2 A}{(x-y)^{2}}\left(C_{0}(x+y)+C_{1}\left(x^{2}+y^{2}\right)+C_{2} x y(x+y)+2 C_{3} x^{2} y^{2}\right) .
\end{aligned}
$$

Remark 3. For example, $\left(\left\langle t^{-2}\right\rangle, 0 ;\left\langle t, t^{2}, t^{3}, t^{4}\right\rangle\right)$ in the above (Rat- $A_{1}$-bry) means

$$
u^{-}(t)=A t^{-2}, \quad u^{+}(t)=0, \quad v(t)=w(t)=C_{0} t+C_{1} t^{2}+C_{2} t^{3}+C_{3} t^{4}
$$

with a convention similar to that in (4.5).

We will review the proof of the above theorem after certain remarks.

Remark 4. All the invariant integrable potentials of type $B_{2}$ together with $P_{2}$ are determined by $[24,25]$. They are classified into three cases. In the normal case they are (Ellip- $\left.B_{2}\right)$, (Trig- $\left.B_{2}\right)$ and (Rat- $B_{2}$ ) which have the following unified expression of the invariant potentials given by [32, Lemma 7.3], where the periods $2 \omega_{1}$ and $2 \omega_{2}$ may be infinite (cf. (2.6) and (2.7))

$$
R(x, y)=A \wp(x-y)+A \wp(x+y)+\frac{C_{4} \wp(x)^{4}+C_{3} \wp(x)^{3}+C_{2} \wp(x)^{2}+C_{1} \wp(x)+C_{0}}{\wp^{\prime}(x)^{2}}
$$




$$
\begin{aligned}
& +\frac{C_{4} \wp(y)^{4}+C_{3} \wp(y)^{3}+C_{2} \wp(y)^{2}+C_{1} \wp(y)+C_{0}}{\wp^{\prime}(y)^{2}}, \\
T(x, y)= & 4 A(\wp(x)-\wp(y))^{-2}\left(C_{4} \wp(x)^{2} \wp(y)^{2}+\frac{C_{3}}{2} \wp(x)^{2} \wp(y)\right. \\
& \left.+\frac{C_{3}}{2} \wp(x) \wp(y)^{2}+C_{2} \wp(x) \wp(y)+\frac{C_{1}}{2} \wp(x)+\frac{C_{1}}{2} \wp(y)+C_{0}\right) .
\end{aligned}
$$

This is the original form we found in the classification of the invariant integrable systems of type $B_{n}$ (cf. [25]). Later we knew Inozemtzev model and in fact, when the periods are finite, (2.3) and (2.5) show that the above potential function corresponds to (Ellip- $B_{2}$ ).

When $\omega_{1}=\omega_{2}=\infty, \wp(t)=t^{-2}$ and $(\wp(x)-\wp(y))^{-2}=x^{4} y^{4}\left(x^{2}-y^{2}\right)^{-2}$ and

$$
\begin{aligned}
R(x, y)= & \frac{A}{(x-y)^{2}}+\frac{A}{(x+y)^{2}}+\frac{1}{4}\left(C_{4} x^{-2}+C_{3}+C_{2} x^{2}+C_{1} x^{4}+C_{0} x^{6}\right) \\
& +\frac{1}{4}\left(C_{4} y^{-2}+C_{3}+C_{2} y^{2}+C_{1} y^{4}+C_{0} y^{6}\right), \\
T(x, y)= & 2 A\left(x^{2}-y^{2}\right)^{-2}\left(2 C_{4}+C_{3}\left(x^{2}+y^{2}\right)+2 C_{2} x^{2} y^{2}+C_{1} x^{2} y^{2}\left(x^{2}+y^{2}\right)+2 C_{0} x^{4} y^{4}\right) .
\end{aligned}
$$

We review these invariant cases discussed in [24, 32]. Owing to the identity

$$
\begin{aligned}
& 2\left(u^{-}-u^{+}\right) v^{\prime}+4 v\left(\left(u^{-}\right)^{\prime}-\left(u^{+}\right)^{\prime}\right)+\partial_{y}\left(2\left(u^{-}+u^{+}\right)(v+w)-4 v w\right) \\
& \quad=2\left|\begin{array}{ccc}
v & v^{\prime} & 1 \\
w & -w^{\prime} & 1 \\
u^{-} & -\left(u^{-}\right)^{\prime} & 1
\end{array}\right|+2\left|\begin{array}{ccc}
v & -v^{\prime} & 1 \\
w & -w^{\prime} & 1 \\
u^{+} & \left(u^{+}\right)^{\prime} & 1
\end{array}\right|
\end{aligned}
$$

and (2.9), the right hand side of the above is zero and we have (4.3) when

$$
u^{-}=C \wp(x-y)+C^{\prime}, \quad u^{+}=C \wp(x+y)+C^{\prime}, \quad v=C \wp(x)+C^{\prime} \quad \text { and } \quad w=C \wp(y)+C^{\prime}
$$

with $T(x, y)=2\left(u^{-}+u^{+}\right)(v+w)-4 v w$. Hence with $Q(\wp(t) ; \wp(t))=\wp(x) \wp(y)$, the function $T(x, y)$ given by (4.4) satisfies (4.3) with the above $u^{ \pm}, v$ and $w$. Using the transformations $(x, y) \mapsto\left(x+\omega_{j}, y+\omega_{j}\right)$, we have

$$
\wp\left(x+\omega_{j}\right) \wp\left(y+\omega_{j}\right)=Q\left(\wp\left(t+\omega_{j}\right) ; \wp\left(t+\omega_{j}\right)\right)=Q\left(\wp(t) ; \wp\left(t+\omega_{j}\right)\right)
$$

for $j=0,1,2,3$ because the function $\wp(x \pm y)$ does not change under these transformations (cf. (2.1)). Thus we have Theorem 2 for $\left(\right.$ Ellip- $\left.B_{2}\right)$ in virtue of Lemma 3 and Lemma 4.

Here we note that $\wp$ may be replaced by $\wp_{0}$.

By the limit under $\omega_{2} \rightarrow \infty$, we have the following (Trig- $B_{2}$ ) from (Ellip- $B_{2}$ ). See the proof of [29, Proposition 6.1] for the precise argument.

$\left(\right.$ Trig- $\left.B_{2}\right)$ :

$$
\begin{aligned}
& Q\left(\sinh ^{-2} \lambda t ; \sinh ^{-2} \lambda t\right)=\sinh ^{-2} \lambda x \cdot \sinh ^{-2} \lambda y \\
& Q\left(\sinh ^{-2} \lambda t ; \cosh ^{-2} \lambda t\right)=-\cosh ^{-2} \lambda x \cdot \cosh ^{-2} \lambda y, \\
& Q\left(\sinh ^{-2} \lambda t ; \sinh ^{2} \lambda t\right)=0, \\
& Q\left(\sinh ^{-2} \lambda t ; \frac{1}{4} \sinh ^{2} 2 \lambda t\right)=\sinh ^{2} \lambda x+\sinh ^{2} \lambda y+2 \sinh ^{2} \lambda x \cdot \sinh ^{2} \lambda y .
\end{aligned}
$$

The equations (4.7), (4.8) and (4.9) correspond to (2.14), (2.15) and (2.17), respectively. Moreover (2.8) should be noted and (4.10) corresponds to (2.17) with replacing $\left(\omega_{1}, \lambda\right)$ by $\left(\omega_{1} / 2,2 \lambda\right)$. 
By the limit under $\lambda \rightarrow 0$, we have the following (Rat- $\left.B_{2}\right)$ from (Trig- $B_{2}$ ) as was shown in the proof of [29, Proposition 6.3]. Here we note (2.13) and

$$
\begin{aligned}
& \cosh ^{-2} \lambda t \cdot \sinh ^{2} \lambda t=1-\cosh ^{-2} \lambda t, \\
& \cosh ^{-2} \lambda t \cdot \sinh ^{4} \lambda t=-1+\cosh ^{-2} \lambda t+\sinh ^{2} \lambda t, \\
& \cosh ^{-2} \lambda t \cdot \sinh ^{6} \lambda t=1-\cosh ^{-2} \lambda t-2 \sinh ^{2} \lambda t+\frac{1}{4} \sinh ^{2} 2 \lambda t, \\
& \lim _{\lambda \rightarrow 0} \lambda^{-2 j} \cosh ^{-2} \lambda t \cdot \sinh ^{2 j} \lambda t=t^{2 j} \quad \text { for } \quad j=1,2,3, \\
& \frac{1}{(x-y)^{2}}+\frac{1}{(x+y)^{2}}=\frac{2\left(x^{2}+y^{2}\right)}{\left(x^{2}-y^{2}\right)^{2}} .
\end{aligned}
$$

The result is as follows.

$\left(\right.$ Rat- $\left.B_{2}\right)$ :

$$
\begin{aligned}
Q\left(t^{-2} ; t^{-2}\right) & =x^{-2} y^{-2}, \\
T\left(t^{-2} ; t^{-2}\right) & =4\left(x^{-2}+y^{-2}\right)\left((x-y)^{-2}+(x+y)^{-2}\right)-4 x^{-2} y^{-2} \\
& =\frac{4\left(x^{2}+y^{2}\right)^{2}-4\left(x^{2}-y^{2}\right)^{2}}{x^{2} y^{2}\left(x^{2}-y^{2}\right)^{2}}=\frac{16}{\left(x^{2}-y^{2}\right)^{2}}, \\
Q\left(t^{-2} ; t^{2}\right) & =0, \\
T\left(t^{-2} ; t^{2}\right) & =\frac{4\left(x^{2}+y^{2}\right)^{2}}{\left(x^{2}-y^{2}\right)^{2}}=\frac{16 x^{2} y^{2}}{\left(x^{2}-y^{2}\right)^{2}}+4, \\
Q\left(t^{-2} ; t^{4}\right) & =x^{2}+y^{2}, \\
T\left(t^{-2}, t^{4}\right) & =\frac{4\left(x^{2}+y^{2}\right)\left(x^{4}+y^{4}\right)}{\left(x^{2}-y^{2}\right)^{2}}-4\left(x^{2}+y^{2}\right)=\frac{8 x^{2} y^{2}\left(x^{2}+y^{2}\right)}{\left(x^{2}-y^{2}\right)^{2}}, \\
Q\left(t^{-2} ; t^{6}\right) & =x^{4}+y^{4}+3 x^{2} y^{2}, \\
T\left(t^{-2}, t^{6}\right) & =\frac{4\left(x^{2}+y^{2}\right)\left(x^{6}+y^{6}\right)}{\left(x^{2}-y^{2}\right)^{2}}-4\left(x^{4}+3 x^{2} y^{2}+y^{4}\right)=\frac{16 x^{4} y^{4}}{\left(x^{2}-y^{2}\right)^{2}} .
\end{aligned}
$$

This expression of $T(x, y)$ for (Rat- $\left.B_{2}\right)$ is also given in Remark 4. Note that we ignore the difference of constants for $Q$ and $T$.

Proof of Theorem 2. The three cases (Ellip- $\left.B_{2}\right)$, (Trig- $\left.B_{2}\right)$ and (Rat- $\left.B_{2}\right)$ have been explained. Note that if $u^{ \pm}, v, w$ and $T\left(u^{-}, u^{+} ; v, w\right)$ (or $\left.Q\left(u^{-}, u^{+} ; v, w\right)\right)$ are defined and they have an analytic parameter, Lemma 3 assures that their analytic continuations also define $P$ and $P_{2}$ satisfying $\left[P, P_{2}\right]=0$.

(Toda- $D_{2}^{(1)}$-bry $\leftarrow$ (Ellip- $\left.B_{2}\right)$ : Replacing $(x, y)$ by $\left(x+\omega_{2}, y\right)$, we have

$$
\begin{aligned}
& Q\left(\cosh 2 \lambda t ; 0, \sinh ^{-2} \lambda t\right)=\lim _{\omega_{2} \rightarrow \infty} Q\left(\frac{e^{2 \lambda \omega_{2}}}{8 \lambda^{2}} \wp_{0}\left(t+\omega_{2}\right) ; \frac{1}{\lambda^{2}} \wp_{0}\left(t+\omega_{2}\right), \frac{1}{\lambda^{2}} \wp_{0}(t)\right) \\
& =\lim _{\omega_{2} \rightarrow \infty} \frac{e^{2 \lambda \omega_{2}}}{8 \lambda^{4}} \wp_{0}\left(x+\omega_{2}\right) \wp_{0}(y)=\cosh 2 \lambda x \cdot \sinh ^{-2} \lambda y, \\
& Q\left(\cosh 2 \lambda t ; 0, \cosh ^{-2} \lambda t\right)=\lim _{\omega_{2} \rightarrow \infty} Q\left(\frac{e^{2 \lambda \omega_{2}}}{8 \lambda^{2}} \wp_{0}\left(t+\omega_{2}\right) ;-\frac{1}{\lambda^{2}} \wp_{0}\left(t+\omega_{1}+\omega_{2}\right) ;-\frac{1}{\lambda_{2}} \wp_{0}\left(t+\omega_{1}\right)\right) \\
& =-\lim _{\omega_{2} \rightarrow \infty} \frac{e^{2 \lambda \omega_{2}}}{8 \lambda^{4}} \wp_{0}\left(x+\omega_{1}+\omega_{2}\right) \cdot \wp_{0}\left(y+\omega_{1}\right) \\
& =-\cosh 2 \lambda x \cdot \cosh ^{-2} \lambda y, \\
& Q\left(\cosh 2 \lambda t ; \sinh ^{-2} \lambda t, 0\right)=\sinh ^{-2} \lambda x \cdot \cosh ^{2} \lambda y,
\end{aligned}
$$


$Q\left(\cosh 2 \lambda t ; \cosh ^{-2} \lambda t, 0\right)=-\cosh ^{-2} \lambda x \cdot \sinh ^{2} \lambda y$.

Hence

$T\left(\cosh 2 \lambda t ; 0, \sinh ^{-2} \lambda t\right)=2(\cosh 2 \lambda(x+y)+\cosh 2 \lambda(x-y)) \cdot \sinh ^{-2} \lambda y$

$$
-4 \cosh 2 \lambda x \cdot \sinh ^{-2} \lambda y=8 \cosh 2 \lambda x,
$$

$T\left(\cosh 2 \lambda t ; 0, \cosh ^{-2} \lambda t\right)=2(\cosh 2 \lambda(x+y)+\cosh 2 \lambda(x-y)) \cdot \cosh ^{-2} \lambda y$

$+4 \cosh 2 \lambda x \cdot \cosh ^{-2} \lambda y=8 \cosh 2 \lambda x$,

$T\left(\cosh 2 \lambda t ; 0, \sinh ^{-2} 2 \lambda t\right)=T\left(\cosh 2 \lambda t ; \sinh ^{-2} 2 \lambda t, 0\right)=0$,

$T\left(\cosh 2 \lambda t ; \sinh ^{-2} \lambda t, 0\right)=8 \cosh 2 \lambda y$.

(Toda- $B_{2}^{(1)}$-bry) $\leftarrow\left(\right.$ Toda- $D_{2}^{(1)}$-bry): Replacing $(x, y)$ by $(x-N, y)$, we have

$$
\begin{aligned}
& \begin{aligned}
T\left(e^{-2 \lambda t} ; 0, \sinh ^{-2} \lambda t\right) & =\lim _{N \rightarrow \infty} T\left(2 e^{-2 \lambda N} \cosh 2 \lambda(t-N) ; 0, \sinh ^{-2} \lambda t\right) \\
& =\lim _{N \rightarrow \infty} 16 e^{-2 \lambda N} \cosh 2 \lambda(x-N)=8 e^{-2 \lambda x},
\end{aligned} \\
& T\left(e^{-2 \lambda t} ; 0, \sinh ^{-2} 2 \lambda t\right)=\lim _{N \rightarrow \infty} T\left(2 e^{-2 \lambda N} \cosh 2 \lambda(t-N) ; 0, \sinh ^{-2} 2 \lambda t\right)=0, \\
& T\left(e^{-2 \lambda t} ; e^{2 \lambda t}, 0\right)=\lim _{N \rightarrow \infty} T\left(2 e^{-2 \lambda N} \cosh 2 \lambda(t-N) ; \frac{1}{4} e^{2 \lambda N} \sinh ^{-2} \lambda(t-N), 0\right)=4 \cosh 2 \lambda y, \\
& T\left(e^{-2 \lambda t} ; e^{4 \lambda t}, 0\right)=\lim _{N \rightarrow \infty} T\left(2 e^{-2 \lambda N} \cosh 2 \lambda(t-N) ; \frac{1}{4} e^{4 \lambda N} \sinh ^{-2} 2 \lambda(t-N), 0\right)=0 .
\end{aligned}
$$

$\left(\operatorname{Trig}-C_{2}^{(1)}\right) \leftarrow\left(\right.$ Trig- $B_{2}^{(1)}$-bry): Replacing $(x, y)$ by $(x+N, y+N)$,

$$
\begin{aligned}
T\left(e^{-2 \lambda t}, 0 ; e^{2 \lambda t}, 0\right) & =\lim _{N \rightarrow \infty} T\left(e^{-2 \lambda t}, e^{-2 \lambda(t+2 N)} ; e^{-2 \lambda N} e^{2 \lambda(t+N)}, 0\right) \\
& =\lim _{N \rightarrow \infty} e^{-2 \lambda N} \cosh 2 \lambda(y+N)=2 e^{2 \lambda y}, \\
T\left(e^{-2 \lambda t}, 0 ; e^{4 \lambda t}, 0\right) & =\lim _{N \rightarrow \infty} T\left(e^{-2 \lambda t}, e^{-2 \lambda(t+2 N)} ; e^{-4 \lambda N} e^{4 \lambda(t+N)}, 0\right)=0 .
\end{aligned}
$$

By the transformation $(x, y, \lambda) \mapsto(y, x,-\lambda)$, we have

$$
T\left(e^{-2 \lambda t}, 0 ; 0, e^{-2 \lambda t}\right)=2 e^{-2 \lambda x}, \quad T\left(e^{-2 \lambda t}, 0 ; 0, e^{-4 \lambda t}\right)=0 .
$$

(Trig- $A_{1}^{(1)}$-bry $) \leftarrow\left(\right.$ Trig- $\left.B_{2}\right):$ Replacing $(x, y)$ by $(x+N, y+N)$,

$$
\begin{aligned}
Q\left(\sinh ^{-2} \lambda t, 0 ; e^{2 \lambda t}\right)= & \lim _{N \rightarrow \infty} Q\left(\sinh ^{-2} \lambda t, \sinh ^{-2} \lambda(t+2 N) ; \frac{1}{4} e^{2 \lambda N} \sinh ^{-2} \lambda(t+N)\right) \\
= & \lim _{N \rightarrow \infty} \frac{1}{4} e^{2 \lambda N} \sinh ^{-2} \lambda(x+N) \sinh ^{-2} \lambda(y+N)=0, \\
T\left(\sinh ^{-2} \lambda t, 0 ; e^{2 \lambda t}\right)= & 2\left(e^{2 \lambda x}+e^{2 \lambda y}\right) \sinh ^{-2} \lambda(x-y), \\
Q\left(\sinh ^{-2} \lambda t, 0 ; e^{4 \lambda t}\right) \lim _{N \rightarrow \infty} Q\left(\sinh ^{-2} \lambda t, \sinh ^{-2} \lambda(t+2 N) ; 4 e^{-4 \lambda N} \sinh ^{2} 2 \lambda(t+N)\right) & =16 \lim _{N \rightarrow \infty} e^{-4 \lambda N}\left(\sinh ^{2} \lambda(x+N)+\sinh ^{2} \lambda(y+N)\right. \\
& \left.+2 \sinh ^{2} \lambda(x+N) \sinh ^{2} \lambda(y+N)\right)=2 e^{2 \lambda(x+y)}, \\
T\left(\sinh ^{-2} \lambda t, 0 ; e^{4 \lambda t}\right)= & 2 \sinh ^{-2} \lambda(x-y)\left(e^{4 \lambda x}+e^{4 \lambda y}\right)-8 e^{2 \lambda(x+y)} \\
= & 2 \sinh ^{-2} \lambda(x-y)\left(e^{4 \lambda x}+e^{4 \lambda y}-e^{2 \lambda(x+y)}\left(e^{\lambda(x-y)}-e^{-\lambda(x-y)}\right)^{2}\right) \\
= & 4 e^{2 \lambda(x+y)} \sinh ^{-2} \lambda(x-y) .
\end{aligned}
$$


(Rat- $A_{1}$-bry $) \leftarrow$ (Trig- $A_{1}$-bry): Taking the limit $\lambda \rightarrow 0$,

$$
\begin{aligned}
Q\left(t^{-2}, 0 ; t\right) & =\lim _{\lambda \rightarrow 0} Q\left(\lambda^{2} \sinh ^{-2} \lambda t, 0 ; \frac{1}{2 \lambda}\left(e^{2 \lambda t}-1\right)\right)=0, \\
T\left(t^{-2}, 0 ; t\right) & =\frac{2(x+y)}{(x-y)^{2}}, \\
Q\left(t^{-2}, 0 ; t^{2}\right) & =\lim _{\lambda \rightarrow 0} Q\left(\lambda^{2} \sinh ^{-2} \lambda t, 0 ; \frac{1}{4 \lambda^{2}}\left(e^{2 \lambda t}+e^{-2 \lambda t}-2\right)\right)=0, \\
T\left(t^{-2}, 0 ; t^{2}\right) & =2 \frac{x^{2}+y^{2}}{(x-y)^{2}}, \\
Q\left(t^{-2}, 0 ; t^{3}\right) & =\lim _{\lambda \rightarrow 0} Q\left(\lambda^{2} \sinh ^{-2} \lambda t, 0 ; \frac{1}{8 \lambda^{3}}\left(e^{4 \lambda t}-3 e^{2 \lambda t}-e^{-2 \lambda t}+3\right)\right) \\
& =\lim _{\lambda \rightarrow 0} \frac{2}{8 \lambda}\left(e^{2 \lambda(x+y)}-1\right)=\frac{1}{2}(x+y), \\
T\left(t^{-2}, 0 ; t^{3}\right) & =2 \frac{x^{3}+y^{3}}{(x-y)^{2}}-2(x+y)=2 \frac{x y(x+y)}{(x-y)^{2}}, \\
Q\left(t^{-2}, 0 ; t^{4}\right) & =\lim _{\lambda \rightarrow 0} Q\left(\lambda^{2} \sinh ^{-2} \lambda t, 0 ; \frac{1}{16 \lambda^{4}}\left(e^{4 \lambda t}+e^{-4 \lambda t}-4 e^{2 \lambda t}-4 e^{-2 \lambda t}+6\right)\right) \\
& =\lim _{\lambda \rightarrow 0} \frac{2}{16 \lambda^{2}}\left(e^{2 \lambda(x+y)}+e^{-2 \lambda(x+y)}-2\right)=\frac{1}{2}(x+y)^{2}, \\
T\left(t^{-2}, 0 ; t^{4}\right) & =2 \frac{x^{4}+y^{4}}{(x-y)^{2}}-2(x+y)^{2}=\frac{4 x^{2} y^{2}}{(x-y)^{2}} .
\end{aligned}
$$

Thus we have completed the proof of Theorem 2 by using Lemma 3 and Lemma 4 .

Remark 5. Theorem 2 can be checked by direct calculations. For example, Remark 2 and the equations

$$
\begin{gathered}
2\left(\varepsilon e^{-2 \lambda(x+y)}-e^{-2 \lambda(x-y)}\right)\left(e^{2 \lambda x}\right)^{\prime}+4 e^{2 \lambda x} \frac{\partial}{\partial x}\left(\varepsilon e^{-2 \lambda(x+y)}-e^{-2 \lambda(x-y)}\right) \\
=4 \lambda\left(\varepsilon e^{-2 \lambda y}-e^{2 \lambda y}\right)-8 \lambda\left(\varepsilon e^{-2 \lambda y}-e^{2 \lambda y}\right)=\frac{\partial}{\partial y}\left(2\left(\varepsilon e^{-2 \lambda y}+e^{2 \lambda y}\right)\right) \\
2\left(\varepsilon e^{-2 \lambda(x+y)}-e^{-2 \lambda(x-y)}\right)\left(e^{4 \lambda x}\right)^{\prime}+4 e^{4 \lambda x} \frac{\partial}{\partial x}\left(\varepsilon e^{-2 \lambda(x+y)}-e^{-2 \lambda(x-y)}\right)=0
\end{gathered}
$$

with $\varepsilon=1$ give $T\left(e^{-2 \lambda t} ; e^{2 \lambda t}, 0\right)$ and $T\left(e^{-2 \lambda t} ; e^{4 \lambda t}, 0\right)$ for (Trig- $B_{2}$-bry). Moreover the functions $T\left(e^{-2 \lambda t}, 0 ; e^{2 \lambda t}, 0\right)$ and $T\left(e^{-2 \lambda t}, 0 ; e^{4 \lambda t}, 0\right)$ for (Toda- $\left.C_{2}^{(1)}\right)$ also follow from these equations with $\varepsilon=0$.

\subsection{Special case}

In this subsection we study the integrable systems (4.1) with (4.2) which are of the form

$$
\begin{aligned}
& R(x, y)=u^{-}(x-y)+u^{+}(x+y)+v(x)+w(y), \\
& u^{-}(t)=\sum_{j=0}^{1} A_{j} u_{j}^{-}(t), \quad u^{+}(t)=\sum_{j=0}^{1} A_{j} u_{j}^{+}(t), \\
& v(t)=\sum_{j=0}^{1} C_{j} v_{j}(t), \quad w(t)=\sum_{j=0}^{1} C_{j} w_{j}(t)
\end{aligned}
$$

with $A_{0}, A_{1}, C_{0}, C_{1} \in \mathbb{C}$. The most general system (Ellip- $B_{2}-\mathrm{S}$ ) in the following theorem is presented by [24] as an elliptic generalization of (Trig- $\left.B_{2}-\mathrm{S}\right)$ found by [32]. 
Theorem 3 ( $B_{2}$ : Special case, $[23,24,30]$ etc.). The operators $P$ and $P_{2}$ defined by the following pairs of $R(x, y)$ and $T(x, y)$ satisfy (4.1) and (4.2).

(Ellip- $\left.B_{2}-\mathrm{S}\right): \quad\left(\left\langle\wp\left(t ; 2 \omega_{1}, 2 \omega_{2}\right), \wp\left(t ; \omega_{1}, 2 \omega_{2}\right)\right\rangle ;\left\langle\wp\left(t ; \omega_{1}, 2 \omega_{2}\right), \wp\left(t ; \omega_{1}, \omega_{2}\right)\right\rangle\right)$

$$
\begin{aligned}
& v(x)=C_{0} \wp\left(x ; \omega_{1}, 2 \omega_{2}\right)+C_{1} \wp\left(x ; \omega_{1}, \omega_{2}\right), \quad w(y)=C_{0} \wp\left(y ; \omega_{1}, 2 \omega_{2}\right)+C_{1} \wp\left(y ; \omega_{1}, \omega_{2}\right), \\
& u^{-}(x-y)=A_{0} \wp\left(x-y ; 2 \omega_{1}, 2 \omega_{2}\right)+A_{1 \wp}\left(x-y ; \omega_{1}, 2 \omega_{2}\right) \text {, } \\
& u^{+}(x+y)=A_{0} \wp\left(x+y ; 2 \omega_{1}, 2 \omega_{2}\right)+A_{1 \wp}\left(x+y ; \omega_{1}, 2 \omega_{2}\right) \text {, } \\
& R(x, y)=A_{0} \wp\left(x-y ; 2 \omega_{1}, 2 \omega_{2}\right)+A_{0} \wp\left(x+y ; 2 \omega_{1}, 2 \omega_{2}\right) \\
& +A_{1} \wp\left(x-y ; \omega_{1}, 2 \omega_{2}\right)+A_{1} \wp\left(x+y ; \omega_{1}, 2 \omega_{2}\right) \\
& +C_{0 \wp}\left(x ; \omega_{1}, 2 \omega_{2}\right)+C_{0} \wp\left(y ; \omega_{1}, 2 \omega_{2}\right)+C_{1 \wp}\left(x ; \omega_{1}, \omega_{2}\right)+C_{1} \wp\left(y ; \omega_{1}, \omega_{2}\right), \\
& T(x, y)=2\left(A_{0 \wp}\left(x-y ; 2 \omega_{1}, 2 \omega_{2}\right)+A_{0} \wp\left(x+y ; 2 \omega_{1}, 2 \omega_{2}\right)\right. \\
& \left.+A_{1 \wp}\left(x-y ; \omega_{1}, 2 \omega_{2}\right)+A_{1 \wp}\left(x+y ; \omega_{1}, 2 \omega_{2}\right)\right) \\
& \times\left(C_{0} \wp\left(x ; \omega_{1}, 2 \omega_{2}\right)+C_{0} \wp\left(y ; \omega_{1}, 2 \omega_{2}\right)+C_{1 \wp}\left(x ; \omega_{1}, \omega_{2}\right)+C_{1} \wp\left(y ; \omega_{1}, \omega_{2}\right)\right) \\
& -4 A_{0} C_{0} \sum_{j=0}^{1} \wp\left(x+\omega_{j} ; 2 \omega_{1}, 2 \omega_{2}\right) \cdot \wp\left(y+\omega_{j} ; 2 \omega_{1}, 2 \omega_{2}\right) \\
& -4 A_{0} C_{1} \sum_{j=0}^{3} \wp\left(x+\omega_{j} ; 2 \omega_{1}, 2 \omega_{2}\right) \wp\left(y+\omega_{j} ; 2 \omega_{1}, 2 \omega_{2}\right) \\
& -4 A_{1} C_{0} \wp\left(x ; \omega_{1}, 2 \omega_{2}\right) \wp\left(y ; \omega_{1}, 2 \omega_{2}\right) \\
& -4 A_{1} C_{1} \sum_{j=0}^{1} \wp\left(x+\omega_{2 j} ; \omega_{1}, 2 \omega_{2}\right) \wp\left(y+\omega_{2 j} ; \omega_{1}, 2 \omega_{2}\right) \text {. }
\end{aligned}
$$

(Trig- $\left.B_{2}-\mathrm{S}\right): \quad\left(\left\langle\sinh ^{-2} \lambda t, \sinh ^{-2} 2 \lambda t\right\rangle ;\left\langle\sinh ^{-2} 2 \lambda t, \sinh ^{2} 2 \lambda t\right\rangle\right)$

$$
\begin{aligned}
v(x)=C_{0} & \sinh ^{-2} 2 \lambda x+C_{1} \sinh ^{2} 2 \lambda x, \quad w(y)=C_{0} \sinh ^{-2} 2 \lambda y+C_{1} \sinh ^{2} 2 \lambda y, \\
u^{-}(x-y) & =A_{0} \sinh ^{-2} \lambda(x-y)+A_{1} \sinh ^{-2} 2 \lambda(x-y), \\
u^{+}(x+y) & =A_{0} \sinh ^{-2} \lambda(x+y)+A_{1} \sinh ^{-2} 2 \lambda(x+y), \\
R(x, y)= & A_{0} \sinh ^{-2} \lambda(x+y)+A_{0} \sinh ^{-2} \lambda(x-y)+A_{1} \sinh ^{-2} 2 \lambda(x+y) \\
& +A_{1} \sinh ^{-2} 2 \lambda(x-y)+C_{0} \sinh ^{-2} 2 \lambda x+C_{0} \sinh ^{-2} 2 \lambda y \\
& +C_{1} \sinh ^{2} 2 \lambda x+C_{1} \sinh ^{2} 2 \lambda y, \\
T(x, y)= & 2\left(A_{0} \sinh ^{-2} \lambda(x+y)+A_{0} \sinh ^{-2} \lambda(x-y)+A_{1} \sinh ^{-2} 2 \lambda(x+y)\right. \\
& \left.+A_{1} \sinh ^{-2} 2 \lambda(x-y)\right)\left(C_{0} \sinh ^{-2} 2 \lambda x+C_{0} \sinh ^{-2} 2 \lambda y+C_{1} \sinh ^{2} 2 \lambda x\right. \\
& \left.+C_{1} \sinh ^{2} 2 \lambda y\right)-A_{0} C_{0}\left(\sinh ^{-2} \lambda x \cdot \sinh ^{-2} \lambda y+\cosh ^{-2} \lambda x \cdot \cosh ^{-2} \lambda y\right) \\
& -4 A_{0} C_{1}\left(\sinh ^{2} \lambda x+\sinh ^{2} \lambda y+2 \sinh ^{2} \lambda x \cdot \sinh ^{2} \lambda y\right) \\
& -4 A_{1} C_{0} \sinh ^{-2} 2 \lambda x \cdot \sinh ^{-2} 2 \lambda y .
\end{aligned}
$$

(Rat- $B_{2}$-S $): \quad\left(\left\langle t^{-2}, t^{2}\right\rangle ;\left\langle t^{-2}, t^{2}\right\rangle\right)$

$$
\begin{aligned}
& v(x)=C_{0} x^{-2}+C_{1} x^{2}, \quad w(y)=C_{0} y^{-2}+C_{1} y^{2}, \\
& u^{-}(x-y)=\frac{A_{0}}{(x-y)^{2}}+A_{1}(x-y)^{2}, \quad u^{+}(x+y)=\frac{A_{0}}{(x+y)^{2}}+A_{1}(x+y)^{2}, \\
& R(x, y)=\frac{A_{0}}{(x-y)^{2}}+\frac{A_{0}}{(x+y)^{2}}+A_{1}(x-y)^{2}+A_{1}(x+y)^{2}+\frac{C_{0}}{x^{2}}+\frac{C_{0}}{y^{2}}+C_{1} x^{2}+C_{1} y^{2}, \\
& T(x, y)=\frac{16 A_{0} C_{0}+16 A_{0} C_{1} x^{2} y^{2}}{\left(x^{2}-y^{2}\right)^{2}}+16 A_{1} C_{1} x^{2} y^{2} .
\end{aligned}
$$


(Toda- $D_{2}^{(1)}$-S-bry): $\quad\left(\langle\cosh 2 \lambda, \cosh 4 \lambda t\rangle ;\left\langle\sinh ^{-2} 2 \lambda t\right\rangle,\left\langle\sinh ^{-2} 2 \lambda t\right\rangle\right)$

$$
\begin{aligned}
& v(x)=C_{0} \sinh ^{-2} 2 \lambda x, \quad w(y)=C_{1} \sinh ^{-2} 2 \lambda y, \\
& u^{-}(x-y)=A_{0} \cosh 2 \lambda(x-y)+A_{1} \cosh 4 \lambda(x-y), \\
& u^{+}(x+y)=A_{0} \cosh 2 \lambda(x+y)+A_{1} \cosh 4 \lambda(x+y), \\
& R(x, y)=A_{0} \cosh 2 \lambda(x-y)+A_{0} \cosh 2 \lambda(x+y)+A_{1} \cosh 4 \lambda(x-y) \\
& \quad+A_{1} \cosh 4 \lambda(x+y)+C_{0} \sinh ^{-2} 2 \lambda x+C_{1} \sinh ^{-2} 2 \lambda y, \\
& T(x, y)=8 A_{1}\left(C_{0} \cosh 4 \lambda y+C_{1} \cosh 4 \lambda x\right) .
\end{aligned}
$$

(Toda- $B_{2}^{(1)}$-S-bry): $\quad\left(\left\langle e^{-2 \lambda t}, e^{-4 \lambda t}\right\rangle ;\left\langle e^{4 \lambda t}\right\rangle,\left\langle\sinh ^{-2} 2 \lambda t\right\rangle\right)$

$$
\begin{aligned}
& v(x)=C_{0} e^{4 \lambda x}, \quad w(y)=C_{1} \sinh ^{-2} 2 \lambda y, \\
& u^{-}(x-y)=A_{0} e^{-2 \lambda(x-y)}+A_{1} e^{-4 \lambda(x-y)}, \quad u^{+}(x+y)=A_{0} e^{-2 \lambda(x+y)}+A_{1} e^{-4 \lambda(x+y)}, \\
& R(x, y)=A_{0} e^{-2 \lambda(x-y)}+A_{0} e^{-2 \lambda(x+y)}+A_{1} e^{-4 \lambda(x-y)}+A_{1} e^{-4 \lambda(x+y)}+C_{0} e^{4 \lambda x}+C_{1} \sinh ^{-2} 2 \lambda y, \\
& T(x, y)=4 A_{1}\left(C_{0} \cosh 4 \lambda y+2 C_{1} e^{-4 \lambda x}\right) .
\end{aligned}
$$

$\left(\right.$ Toda- $\left.C_{2}^{(1)}-\mathrm{S}\right): \quad\left(\left\langle e^{-2 \lambda t}, e^{-4 \lambda t}\right\rangle, 0 ;\left\langle e^{4 \lambda t}\right\rangle,\left\langle e^{-4 \lambda t}\right\rangle\right)$

$$
\begin{aligned}
& v(x)=C_{0} e^{4 \lambda x}, \quad w(y)=C_{1} e^{-4 \lambda y}, \\
& u^{-}(x-y)=A_{0} e^{-2 \lambda(x-y)}+A_{1} e^{-4 \lambda(x-y)}, \quad u^{+}(x+y)=0, \\
& R(x, y)=A_{0} e^{-2 \lambda(x-y)}+A_{1} e^{-4 \lambda(x-y)}+C_{0} e^{4 \lambda x}+C_{1} e^{-4 \lambda y}, \\
& T(x, y)=2 A_{1}\left(C_{0} e^{4 \lambda y}+C_{1} e^{-4 \lambda x}\right) .
\end{aligned}
$$

(Trig- $A_{1}$-S-bry): $\quad\left(\left\langle\sinh ^{-2} \lambda t, \sinh ^{-2} 2 \lambda t\right\rangle, 0 ;\left\langle e^{-4 \lambda t}, e^{4 \lambda t}\right\rangle\right)$

$$
\begin{aligned}
& v(x)=C_{0} e^{-4 \lambda x}+C_{1} e^{4 \lambda x}, \quad w(y)=C_{0} e^{-4 \lambda y}+C_{1} e^{4 \lambda y}, \\
& u^{-}(x-y)=A_{0} \sinh ^{-2} \lambda(x-y)+A_{1} \sinh ^{-2} 2 \lambda(x-y), \quad u^{+}(x+y)=0, \\
& R(x, y)=A_{0} \sinh ^{-2} \lambda(x-y)+A_{1} \sinh ^{-2} 2 \lambda(x-y)+C_{0} e^{-4 \lambda x}+C_{0} e^{-4 \lambda y}+C_{1} e^{4 \lambda x}+C_{1} e^{4 \lambda y}, \\
& T(x, y)=2 A_{1} \sinh ^{-2} 2 \lambda(x-y)\left(C_{0} e^{-4 \lambda x}+C_{0} e^{-4 \lambda y}+C_{1} e^{4 \lambda x}+C_{1} e^{4 \lambda y}\right) \\
& \quad+4 A_{0} \sinh ^{-2} \lambda(x-y)\left(C_{0} e^{-2 \lambda(x+y)}+C_{1} e^{2 \lambda(x+y)}\right) .
\end{aligned}
$$

$\left(\right.$ Rat $^{d}-D_{2}$-S-bry): $\quad\left(\left\langle t^{2}, t^{4}\right\rangle ;\left\langle t^{-2}\right\rangle,\left\langle t^{-2}\right\rangle\right)$

$$
\begin{aligned}
& v(x)=C_{0} x^{-2}, \quad w(y)=C_{1} y^{-2}, \\
& u^{-}(x-y)=A_{0}(x-y)^{2}+A_{1}(x-y)^{4}, \quad u^{+}(x+y)=A_{0}(x+y)^{2}+A_{1}(x+y)^{4}, \\
& R(x, y)=2 A_{0}\left(x^{2}+y^{2}\right)+2 A_{1}\left(x^{4}+6 x^{2} y^{2}+y^{4}\right)+\frac{C_{0}}{x^{2}}+\frac{C_{1}}{y^{2}}, \\
& T(x, y)=32 A_{1}\left(C_{0} y^{2}+C_{1} x^{2}\right) .
\end{aligned}
$$

Proof. (Ellip- $B_{2}$-S): We have the following from (4.6), Lemma 4 and (2.8).

$$
\begin{aligned}
& Q\left(\wp\left(t ; \omega_{1}, 2 \omega_{2}\right) ; \wp\left(t ; \omega_{1}, 2 \omega_{2}\right)\right)=\wp\left(x ; \omega_{1}, 2 \omega_{2}\right) \wp\left(y ; \omega_{1}, 2 \omega_{2}\right), \\
& Q\left(\wp\left(t ; 2 \omega_{1}, 2 \omega_{2}\right) ; \wp\left(t ; \omega_{1}, 2 \omega_{2}\right)\right)=Q\left(\wp\left(t ; 2 \omega_{1}, 2 \omega_{2}\right) ; \wp\left(t ; 2 \omega_{1}, 2 \omega_{2}\right)\right. \\
& \quad+\wp\left(t 2 A_{1} \sinh ^{-2} 2 \lambda(x-y)\left(C_{0}+\omega_{1} ; 2 \omega_{1}, 2 \omega_{2}\right)\right) \\
& \quad=\wp\left(x ; 2 \omega_{1}, 2 \omega_{2}\right) \wp\left(y ; 2 \omega_{1}, 2 \omega_{2}\right)+\wp\left(x+\omega_{1} ; 2 \omega_{1}, 2 \omega_{2}\right) \wp\left(y+\omega_{1} ; 2 \omega_{1}, 2 \omega_{2}\right), \\
& Q\left(\wp\left(t ; \omega_{1}, 2 \omega_{2}\right) ; \wp\left(t ; \omega_{1}, \omega_{2}\right)\right)=\wp\left(x ; \omega_{1}, 2 \omega_{2}\right) \wp\left(y ; \omega_{1}, 2 \omega_{2}\right)
\end{aligned}
$$




$$
\begin{aligned}
& +\wp\left(x+\omega_{2} ; \omega_{1}, 2 \omega_{2}\right) \wp\left(y+\omega_{2} ; \omega_{1}, 2 \omega_{2}\right), \\
& Q\left(\wp\left(t ; 2 \omega_{1}, 2 \omega_{2}\right) ; \wp\left(t ; \omega_{1}, \omega_{2}\right)\right)=\sum_{j=0}^{3} \wp\left(x+\omega_{j} ; 2 \omega_{1}, 2 \omega_{2}\right) \wp\left(y+\omega_{j} ; 2 \omega_{1}, 2 \omega_{2}\right) .
\end{aligned}
$$

(Rat- $B_{2}-\mathrm{S}$ ) is given in $[32,(7.13)]$ but it is easy to check $(4.3)$ or prove the result as a limit of (Trig- $B_{2}$-S). (Rat ${ }^{d}-D_{2}$-S-bry) follows from $T\left(t^{2} ; t^{-2}, 0\right)=0$ and $T\left(t^{4} ; t^{-2}, 0\right)=32 y^{2}$ (cf. Remark 2 i)). Moreover (Trig- $\left.B_{2}-\mathrm{S}\right)$, (Toda- $D_{2}^{(1)}-\mathrm{S}-$ bry), (Toda- $\left.C_{2}^{(1)}-\mathrm{S}\right)$ and (Trig- $A_{1}$-S-bry) are obtained from the corresponding normal cases together with Lemma 4. For example, $Q$ for (Trig- $\left.B_{2}-\mathrm{S}\right)$ is given by $(4.7),(4.9)$ and

$$
\begin{aligned}
Q\left(\sinh ^{-2} \lambda t ; \sinh ^{-2} 2 \lambda t\right) & =Q\left(\sinh ^{-2} \lambda t ; \frac{1}{4} \sinh ^{-2} \lambda t-\frac{1}{4} \cosh ^{-2} \lambda t\right) \\
& =\frac{1}{4}\left(\sinh ^{-2} \lambda t \sinh ^{-2} \lambda y+\cosh ^{-2} \lambda x \cosh ^{-2} \lambda y\right), \\
Q\left(\sinh ^{-2} 2 \lambda t ; \sinh ^{-2} 2 \lambda t\right) & =\sinh ^{-2} 2 \lambda x \cdot \sinh ^{-2} 2 \lambda y, \\
Q\left(\sinh ^{-2} 2 \lambda t ; \sinh ^{2} 2 \lambda t\right)= & 0 .
\end{aligned}
$$

Thus we get Theorem 3 from Theorem 2 .

\subsection{Duality}

Definition 1 (Duality in $\boldsymbol{B}_{\mathbf{2}},[23]$ ). Under the coordinate transformation

$$
(x, y) \mapsto(X, Y)=\left(\frac{x+y}{\sqrt{2}}, \frac{x-y}{\sqrt{2}}\right)
$$

the pair $\left(P, P^{2}-P_{2}\right)$ also satisfies (4.1), which we call the duality of the commuting differential operators of type $B_{2}$.

Denoting $\partial_{x}=\partial / \partial x, \partial_{y}=\partial / \partial y$ and put

$$
L=P^{2}-P_{2}-\left(\frac{1}{2} \partial_{x}^{2}-\frac{1}{2} \partial_{y}^{2}+w-v\right)^{2}+u^{-}\left(\partial_{x}+\partial_{y}\right)^{2}+u^{+}\left(\partial_{x}-\partial_{y}\right)^{2} .
$$

Then the order of $L$ is at most 2 and the second order term of $L$ is

$$
\begin{aligned}
& -\left(u^{+}+u^{-}+v+w\right)\left(\partial_{x}^{2}+\partial_{y}^{2}\right)-2\left(u^{-}-u^{+}\right) \partial_{x} \partial_{y}+2 w \partial_{x}^{2}+2 v \partial_{y}^{2}-(w-v)\left(\partial_{x}^{2}-\partial_{y}^{2}\right) \\
& +u^{-}\left(\partial_{x}+\partial_{y}\right)^{2}+u^{+}\left(\partial_{x}-\partial_{y}\right)^{2}=0 .
\end{aligned}
$$

Since $L$ is self-adjoint, $L$ is of order at most 0 and the 0 -th order term of $L$ is

$$
\begin{gathered}
-\frac{1}{2}\left(\partial_{x}^{2}+\partial_{y}^{2}\right)\left(u^{+}+u^{-}+v+w\right)+\left(u^{+}+u^{-}+v+w\right)^{2}-4 v w-T-\partial_{x} \partial_{y}\left(u^{-}-u^{+}\right) \\
-\frac{1}{2}\left(\partial_{x}^{2}-\partial_{y}^{2}\right)(w-v)=\left(u^{+}+u^{-}+v+w\right)^{2}-4 v w-T
\end{gathered}
$$

and therefore we have the following proposition.

Proposition $1([23,24])$. i) By the duality in Definition 1 the pair $(R(x, y), T(x, y))$ changes into $(\tilde{R}(x, y), \tilde{T}(x, y))$ with

$$
\tilde{R}(x, y)=v\left(\frac{x+y}{\sqrt{2}}\right)+w\left(\frac{x-y}{\sqrt{2}}\right)+u^{+}(\sqrt{2} x)+u^{-}(\sqrt{2} y),
$$




$$
\tilde{T}(x, y)=\tilde{R}(x, y)^{2}-4 v\left(\frac{x+y}{\sqrt{2}}\right) w\left(\frac{x-y}{\sqrt{2}}\right)-T\left(\frac{x+y}{\sqrt{2}}, \frac{x-y}{\sqrt{2}}\right) .
$$

ii) Combining the duality with the scaling map $R(x, y) \mapsto c^{-2} R(c x, c y)$, the following pair $\left(R^{d}(x, y), T^{d}(x, y)\right)$ defines commuting differential operators if so does $(R(x, y), T(x, y))$. This $R^{d}(x, y)$ is also called the dual of $R(x, y)$,

$$
\begin{aligned}
& R^{d}(x, y)=v(x+y)+w(x-y)+u^{+}(2 x)+u^{-}(2 y), \\
& T^{d}(x, y)=R^{d}(x, y)^{2}-4 v(x+y) w(x-y)-T(x+y, x-y) .
\end{aligned}
$$

Remark 6. i) We list up the systems of type $B_{2}$ given in Sections 4.1 and 4.2:

$$
\begin{aligned}
& \left(u^{-}(t), u^{+}(t) ; v(t), w(t)\right) \\
& \left(\langle\wp(t)\rangle ;\left\langle\wp(t), \wp\left(t+\omega_{1}\right), \wp\left(t+\omega_{2}\right), \wp\left(t+\omega_{3}\right)\right\rangle\right), \\
& \left(\left\langle\wp\left(t ; 2 \omega_{1}, 2 \omega_{2}\right), \wp\left(t ; \omega_{1}, 2 \omega_{2}\right)\right\rangle ;\left\langle\wp\left(t ; \omega_{1}, 2 \omega_{2}\right), \wp\left(t ; \omega_{1}, \omega_{2}\right)\right\rangle\right), \\
& \left(\left\langle\sinh ^{-2} \lambda t\right\rangle ;\left\langle\sinh ^{-2} \lambda t, \sinh ^{-2} 2 \lambda t, \sinh ^{2} \lambda t, \sinh ^{2} 2 \lambda t\right\rangle\right), \\
& \left(\left\langle\sinh ^{-2} \lambda t, \sinh ^{-2} 2 \lambda t\right\rangle ;\left\langle\sinh ^{-2} 2 \lambda t, \sinh ^{2} 2 \lambda t\right\rangle\right), \\
& \left(\left\langle t^{-2}\right\rangle ;\left\langle t^{-2}, t^{2}, t^{4}, t^{6}\right\rangle\right), \\
& \left(\left\langle t^{-2}, t^{2}\right\rangle ;\left\langle t^{-2}, t^{2}\right\rangle\right), \\
& \left(\langle\cosh 2 \lambda t\rangle ;\left\langle\sinh ^{-2} \lambda t, \sinh ^{-2} 2 \lambda t\right\rangle,\left\langle\sinh ^{-2} \lambda t, \sinh ^{-2} 2 \lambda t\right\rangle\right), \\
& \left(\langle\cosh \lambda t, \cosh 2 \lambda t\rangle ;\left\langle\sinh ^{-2} \lambda t\right\rangle,\left\langle\sinh ^{-2} \lambda t\right\rangle\right), \\
& \left(\left\langle e^{-2 \lambda t}\right\rangle ;\left\langle e^{2 \lambda t}, e^{4 \lambda t}\right\rangle,\left\langle\sinh ^{-2} \lambda t, \sinh ^{-2} 2 \lambda t\right\rangle\right), \\
& \left(\left\langle e^{-\lambda t}, e^{-2 \lambda t}\right\rangle ;\left\langle e^{2 \lambda t}\right\rangle,\left\langle\sinh ^{-2} \lambda t\right\rangle\right), \\
& \left(\left\langle e^{-2 \lambda t}\right\rangle, 0 ;\left\langle e^{2 \lambda t}, e^{4 \lambda t}\right\rangle,\left\langle e^{-2 \lambda t}, e^{-4 \lambda t}\right\rangle\right), \\
& \left(\left\langle e^{-2 \lambda t}, e^{-4 \lambda t}\right\rangle, 0 ;\left\langle e^{4 \lambda t}\right\rangle,\left\langle e^{-4 \lambda t}\right\rangle\right), \\
& \left(\left\langle\sinh ^{-2} \lambda t\right\rangle, 0 ;\left\langle e^{-2 \lambda t}, e^{-4 \lambda t}, e^{2 \lambda t}, e^{4 \lambda t}\right\rangle\right), \\
& \left(\left\langle\sinh ^{-2} \lambda t, \sinh ^{-2} 2 \lambda t\right\rangle, 0 ;\left\langle e^{-4 \lambda t}, e^{4 \lambda t}\right\rangle\right), \\
& \left(\left\langle t^{-2}\right\rangle, 0 ;\left\langle t, t^{2}, t^{3}, t^{4}\right\rangle\right), \\
& \left(\left\langle t^{-2}\right\rangle,\left\langle t^{-2}\right\rangle ;\left\langle t^{2}, t^{4}\right\rangle\right) .
\end{aligned}
$$

(Toda- $D_{2}^{(1)}$-bry) (Toda- $D_{2}^{(1)}$-S-bry)$$
\text { (Toda- } B_{2}^{(1)} \text {-bry) }
$$$$
\text { (Toda- } B_{2}^{(1)} \text {-S-bry) }
$$$$
\left(\text { Toda- } C_{2}^{(1)}\right. \text { ) }
$$$$
\text { (Toda- } C_{2}^{(1)}-\mathrm{S} \text { ) }
$$$$
\text { (Trig- } A_{1} \text {-bry) }
$$$$
\text { (Trig- } A_{1} \text {-S-bry) }
$$$$
\text { (Rat- } A_{1} \text {-bry) }
$$$$
\text { (Rat- } D_{2} \text {-S-bry) }
$$

The first 6 cases above are classified by [24] as invariant systems. The systems such that at least two of $u^{ \pm}, v$ and $w$ are not entire are classified by [23]. (Trig-*) and (Toda-*) in the above are classified by [30] as certain systems with periodic potentials.

We do not put $\left(\left\langle t^{-2}, t^{2}\right\rangle, 0 ;\left\langle t, t^{2}\right\rangle\right)$ in the list which corresponds to (Rat- $A_{1}$-S-bry) because its dual defines a direct sum of trivial operators (cf. Section 9$)$.

ii) Since $1-\sinh ^{-2} t+4 \sinh ^{-2} 2 t=\operatorname{coth}^{2} t=t^{2}-(2 / 3) t^{4}+o\left(t^{4}\right)$ and $\sinh ^{2} t=t^{2}+(2 / 3) t^{4}+$ $o\left(t^{4}\right)$, we have $\sinh ^{2} 2 \lambda x+\sinh ^{2} 2 \lambda y-2 \operatorname{coth}^{2} \lambda(x-y)-2 \operatorname{coth}^{2} \lambda(x+y)=8 \lambda^{4}\left(x^{2}+y^{2}\right)^{2}+o\left(\lambda^{4}\right)$. Hence the potential function

$$
\frac{A_{0}}{(x-y)^{2}}+\frac{A_{0}}{(x+y)^{2}}+\frac{C_{0}}{x^{2}}+\frac{C_{0}}{y^{2}}+C_{1}\left(x^{2}+y^{2}\right)+A_{1}\left(x^{2}+y^{2}\right)^{2}
$$

is an analytic continuation of that of $\left(\right.$ Trig- $\left.B_{2}-\mathrm{S}\right)$ but this is not a completely integrable potential function of type $B_{2}$

iii) The dual is indicated by superfix ${ }^{d}$. For example, the dual of (Ellip- $\left.B_{2}\right)$ is denoted by $\left(\right.$ Ellip $\left.^{d}-B_{2}\right)$ whose potential function is

$$
R(x, y)=A \wp(2 x)+A \wp(2 y)+\sum_{j=0}^{3} C_{j}\left(\wp\left(x-y+\omega_{j}\right)+\wp\left(x+y+\omega_{j}\right)\right)
$$


and the dual of (Toda- $\left.C_{2}^{(1)}\right)$ is

$$
\left(\left\langle e^{-2 \lambda t}, e^{-4 \lambda t}\right\rangle,\left\langle e^{2 \lambda t}, e^{4 \lambda t}\right\rangle ; 0,\left\langle e^{-4 \lambda t}\right\rangle\right)
$$

since the dual of $\left(u^{-}(t), u^{+}(t) ; v(t), w(t)\right)$ is $\left(w(t), v(t) ; u^{+}(2 t), u^{-}(2 t)\right)$. Similarly

$$
\left(\left\langle\wp\left(t ; \omega_{1}, 2 \omega_{2}\right), \wp\left(t ; \omega_{1}, \omega_{2}\right)\right\rangle ;\left\langle\wp\left(2 t ; 2 \omega_{1}, 2 \omega_{2}\right), \wp\left(2 t ; \omega_{1}, 2 \omega_{2}\right)\right\rangle\right) .
$$

Since $4 \wp\left(2 t ; 2 \omega_{1}, 2 \omega_{2}\right)=\wp\left(t ; \omega_{1}, \omega_{2}\right)$ etc., $\left(\right.$ Ellip $\left.^{d}-B_{2}-S\right)$ coincides with (Ellip- $\left.B_{2}-S\right)$ by replacing $\left(\omega_{1}, \omega_{2}\right)$ by $\left(2 \omega_{2}, \omega_{1}\right)$.

Then we have the following diagrams and their duals, where the arrows with double lines represent specializations of parameters. For example, "Trig- $B_{2} \stackrel{5: 3}{\Rightarrow}$ Trig- $B C_{2}$-reg" means that 2 parameters (coupling constants $C_{2}$ and $C_{3}$ ) out of 5 in the potential function (Trig- $B_{2}$ ) are specialized to get the potential function (Trig- $B C_{2}$-reg) with 3 parameters. For the normal cases see Definition 5 and the diagrams in the last part of the next section (type $B_{n}$ ).

\section{Hierarchy of Normal Integrable Potentials of type $\boldsymbol{B}_{\mathbf{2}}$}

$$
\begin{aligned}
& \text { Trig- } B C_{2} \text {-reg } \rightarrow \text { Toda- } D_{2} \text {-bry } \\
& C_{2}=C_{3}=0 \Uparrow 5: 3 \quad C_{0}=C_{1}=0 \Uparrow 5: 3 \\
& \text { Trig- } B_{2} \rightarrow \text { Toda- } B_{2}^{(1)} \text {-bry } \underset{C_{0}=C_{1}=0}{\stackrel{5: 3}{\Rightarrow}} \quad \text { Toda- } B_{2}^{(1)}
\end{aligned}
$$

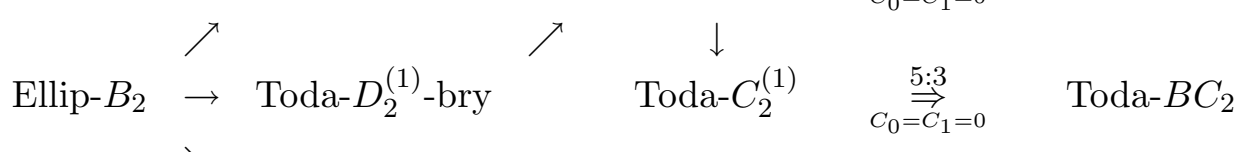

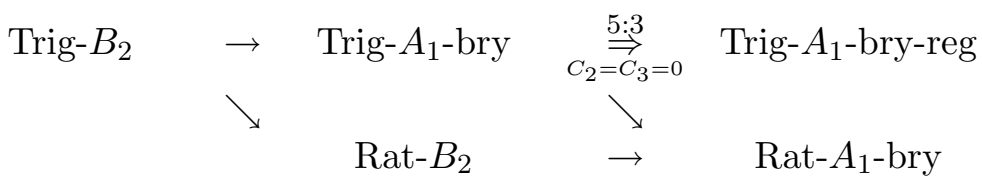

Hierarchy of Special Integrable Potentials of type $\boldsymbol{B}_{\mathbf{2}}$

$$
\begin{aligned}
& \text { Trig }^{(d)}-B_{2} \text {-S-reg } \rightarrow \text { Toda }^{(d)}-D_{2} \text {-S-bry } \quad \text { Rat }^{d} \text { - } D_{2} \text {-S-bry } \\
& C_{1}=0 \text { 个 } 4: 3 \quad C_{0}=0 \text { 个 } 4: 3 \\
& \operatorname{Trig}^{(d)}-B_{2}-\mathrm{S} \quad \rightarrow \text { Toda }^{(d)}-B_{2}^{(1)}-\mathrm{S} \text {-bry } \underset{C_{1}=0}{\stackrel{4: 3}{\Rightarrow}} \quad \operatorname{Toda}^{(d)}-B_{2}^{(1)}-\mathrm{S}
\end{aligned}
$$

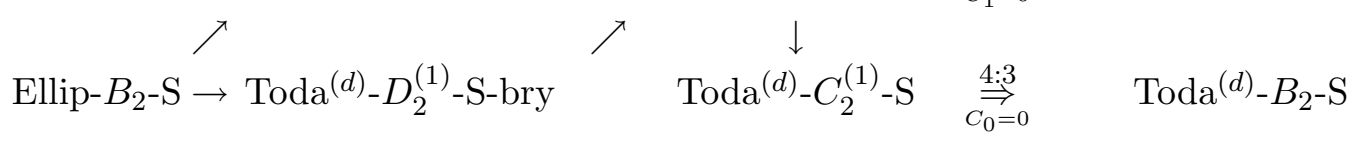

$$
\begin{aligned}
& \operatorname{Trig}^{(d)}-B_{2}-\mathrm{S} \quad \rightarrow \quad \operatorname{Trig}^{(d)}-A_{1} \text {-S-bry } \underset{C_{1}=0}{\stackrel{4: 3}{\Rightarrow}} \operatorname{Trig}^{(d)}-A_{1} \text {-S-bry-reg } \\
& \text { Rat- } B_{2}-\mathrm{S}
\end{aligned}
$$

Definition 2. We define some potential functions as specializations.

(Trig- $B_{2}$-S-reg) Trigonometric special potential of type $B_{2}$ with regular boundary conditions is (Trig- $B_{2}-\mathrm{S}$ ) with $C_{1}=0$.

(Toda- $D_{2}$-S-bry) Toda special potential of type $D_{2}$ with boundary conditions is (Toda- $B_{2}^{(1)}$-S-bry) with $C_{0}=0$.

(Toda- $\left.B_{2}^{(1)}-\mathrm{S}\right)$ Toda special potential of type $B_{2}^{(1)}$ is (Toda- $B_{2}^{(1)}-\mathrm{S}$-bry) with $C_{1}=0$. 
(Toda- $B_{2}-\mathrm{S}$ ) Toda special potential of type $B_{2}$ is (Toda- $C_{2}^{(1)}$-S-bry) with $C_{0}=0$.

(Trig- $A_{1}$-S-bry-reg) Trigonometric special potential of type $A_{1}$ with regular boundary conditions is (Trig- $A_{1}$-S-bry) with $C_{1}=0$.

Remark 7. We have some equivalences as follows:

$$
\begin{aligned}
& \left(\text { Ellip- } B_{2}-\mathrm{S}\right)=\left(\text { Ellip }^{d}-B_{2}-\mathrm{S}\right), \\
& \left(\text { Rat- } B_{2}-\mathrm{S}\right)=\left(\text { Rat }^{d}-B_{2}-\mathrm{S}\right), \\
& \left(\text { Trig- } B C_{2} \text {-reg }\right)=\left(\text { Trig }^{d}-B_{2} \text {-S-reg }\right), \\
& \left(\text { Trig- } A_{1} \text {-bry-reg }\right)=\left(\text { Toda }^{d}-D_{2} \text {-S-bry }\right), \\
& \left(\text { Toda- } D_{2} \text {-bry }\right)=\left(\text { Trig }^{d}-A_{1}-\mathrm{S}-\text { bry-reg }\right), \\
& \left(\text { Toda- } B_{2}^{(1)}\right)=\left(\text { Toda }^{d}-B_{2}^{(1)}-\mathrm{S}\right), \\
& \left(\text { Toda- } B C_{2}\right)=\left(\text { Toda }^{d}-B_{2}-\mathrm{S}\right) .
\end{aligned}
$$

\section{Type $B_{n}(n \geq 3)$}

In this section we construct integrals of the completely integrable systems of type $B_{n}$ appearing in the following diagram. The diagram is also given in [8, Figure III.1], where (Toda- $B_{n}^{(1)}$-bry) is missing. The most general system (Ellip- $\left.B_{n}\right)$ is called Inozemtzev model (cf. [12]).

\section{Hierarchy of Integrable Potentials with 5 parameters $(n \geq 2)$}

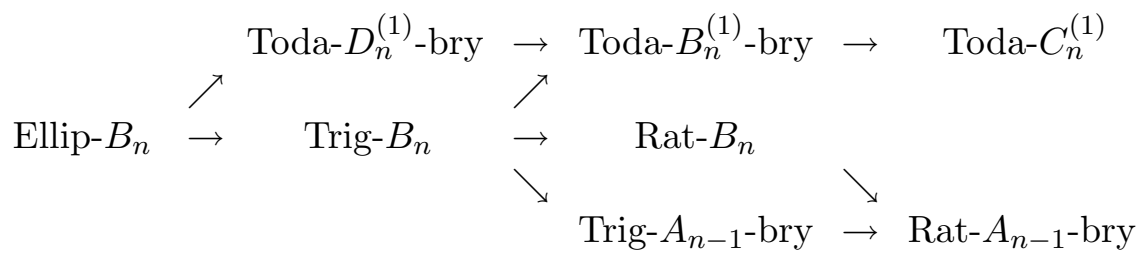

\subsection{Integrable potentials}

Definition 3. The potential functions $R(x)$ of (1.1) are as follows:

Here $A, C_{0}, C_{1}, C_{2}$ and $C_{3}$ are any complex numbers.

(Ellip- $B_{n}$ ) Elliptic potential of type $B_{n}$ :

$$
\sum_{1 \leq i<j \leq n} A\left(\wp\left(x_{i}-x_{j} ; 2 \omega_{1}, 2 \omega_{2}\right)+\wp\left(x_{i}+x_{j} ; 2 \omega_{1}, 2 \omega_{2}\right)\right)+\sum_{k=1}^{n} \sum_{j=0}^{3} C_{j} \wp\left(x_{k}+\omega_{j} ; 2 \omega_{1}, 2 \omega_{2}\right),
$$

(Trig- $B_{n}$ ) Trigonometric potential of type $B_{n}$ :

$$
\begin{aligned}
& \sum_{1 \leq i<j \leq n} A\left(\sinh ^{-2} \lambda\left(x_{i}-x_{j}\right)+\sinh ^{-2} \lambda\left(x_{i}+x_{j}\right)\right) \\
& \quad+\sum_{k=1}^{n}\left(C_{0} \sinh ^{-2} \lambda x_{k}+C_{1} \cosh ^{-2} \lambda x_{k}+C_{2} \sinh ^{2} \lambda x_{k}+\frac{C_{3}}{4} \sinh ^{2} 2 \lambda x_{k}\right),
\end{aligned}
$$

(Rat- $\left.B_{n}\right)$ Rational potential of type $B_{n}$ :

$$
\sum_{1 \leq i<j \leq n}\left(\frac{A}{\left(x_{i}-x_{j}\right)^{2}}+\frac{A}{\left(x_{i}+x_{j}\right)^{2}}\right)+\sum_{k=1}^{n}\left(C_{0} x_{k}^{-2}+C_{1} x_{k}^{2}+C_{2} x_{k}^{4}+C_{3} x_{k}^{6}\right),
$$


(Trig- $A_{n-1}$-bry) Trigonometric potential of type $A_{n-1}$ with boundary conditions:

$$
\sum_{1 \leq i<j \leq n} A \sinh ^{-2} \lambda\left(x_{i}-x_{j}\right)+\sum_{k=1}^{n}\left(C_{0} e^{-2 \lambda x_{k}}+C_{1} e^{-4 \lambda x_{k}}+C_{2} e^{2 \lambda x_{k}}+C_{3} e^{4 \lambda x_{k}}\right),
$$

(Toda- $B_{n}^{(1)}$-bry) Toda potential of type $B_{n}^{(1)}$ with boundary conditions:

$$
\sum_{i=1}^{n-1} A e^{-2 \lambda\left(x_{i}-x_{i+1}\right)}+A e^{-2 \lambda\left(x_{n-1}+x_{n}\right)}+C_{0} e^{2 \lambda x_{1}}+C_{1} e^{4 \lambda x_{1}}+C_{2} \sinh ^{-2} \lambda x_{n}+C_{3} \sinh ^{-2} 2 \lambda x_{n},
$$

(Toda- $\left.C_{n}^{(1)}\right)$ Toda potential of type $C_{n}^{(1)}$ :

$$
\sum_{i=1}^{n-1} A e^{-2 \lambda\left(x_{i}-x_{i+1}\right)}+C_{0} e^{2 \lambda x_{1}}+C_{1} e^{4 \lambda x_{1}}+C_{2} e^{-2 \lambda x_{n}}+C_{3} e^{-4 \lambda x_{n}}
$$

(Toda- $D_{n}^{(1)}$-bry) Toda potential of type $D_{n}^{(1)}$ with boundary conditions:

$$
\begin{aligned}
& \sum_{i=1}^{n-1} A e^{-2 \lambda\left(x_{i}-x_{i+1}\right)}+A e^{-2 \lambda\left(x_{n-1}+x_{n}\right)}+A e^{2 \lambda\left(x_{1}+x_{2}\right)} \\
& \quad+C_{0} \sinh ^{-2} \lambda x_{1}+C_{1} \sinh ^{-2} 2 \lambda x_{1}+C_{2} \sinh ^{-2} \lambda x_{n}+C_{3} \sinh ^{-2} 2 \lambda x_{n},
\end{aligned}
$$

(Rat- $A_{n-1}$-bry) Rational potential of type $A_{n-1}$ with boundary conditions:

$$
\sum_{1 \leq i<j \leq n} \frac{A}{\left(x_{i}-x_{j}\right)^{2}}+\sum_{k=1}^{n}\left(C_{0} x_{k}+C_{1} x_{k}^{2}+C_{2} x_{k}^{3}+C_{3} x_{k}^{4}\right) .
$$

Remark 8. In these cases the Schrödinger operator $P$ is of the form

$$
\begin{aligned}
& P=-\frac{1}{2} \sum_{j=1}^{n} \frac{\partial^{2}}{\partial x_{j}^{2}}+R(x), \\
& R(x)=\sum_{1 \leq i<j \leq n}\left(u_{e_{i}-e_{j}}(x)+u_{e_{i}+e_{j}}(x)\right)+\sum_{j=0}^{3} C_{j} v^{j}(x), \quad v^{j}(x)=\sum_{k=1}^{n} v_{e_{k}}^{j}(x) .
\end{aligned}
$$

Here

$$
\partial_{a} u_{\alpha}(x)=\partial_{b} v_{\beta}^{j}(x)=0 \quad \text { if } \quad a, b \in \mathbb{R}^{n} \quad \text { satisfy } \quad\langle a, \alpha\rangle=\langle b, \beta\rangle=0 .
$$

The complete integrability of the invariant systems (Ellip- $\left.B_{n}\right)$, (Trig- $\left.B_{n}\right)$ and (Rat- $\left.B_{n}\right)$ is established by [29]. We review their integrals and then we prove that the other five systems are also completely integrable by constructing enough integrals, which is announced by [30]. The complete integrability of (Trig- $A_{n-1}$-bry), (Toda- $C_{n}^{(1)}$ ), (Toda- $D_{n}^{(1)}$-bry) and (Rat- $A_{n-1}$-bry) is presented as an unknown problem by [8] and then that of (Toda- $B_{n}^{(1)}$-bry), (Toda- $C_{n}^{(1)}$ ) and (Toda- $D_{n}^{(1)}$-bry) are established by $[18,19,21]$ using $R$-matrix method. The compete integrability of (Trig- $A_{n-1}$-bry) and (Rat- $A_{n-1}$-bry) seems to have not been proved.

Definition $4([25,29])$. Let $u_{\alpha}(x)$ and $T_{I}^{o}\left(v^{j}\right)$ are functions given for $\alpha \in \Sigma\left(D_{n}\right)$ and subsets $I=\left\{i_{1}, \ldots, i_{k}\right\} \subset\{1, \ldots, n\}$ such that

$$
u_{\alpha}(x)=u_{-\alpha}(x) \quad \text { and } \quad \partial_{y} u_{\alpha}=0 \quad \text { for } \quad y \in \mathbb{R}^{n} \quad \text { with } \quad\langle\alpha, y\rangle=0 .
$$


Define a differential operator

$$
P_{n}(C)=\sum_{k=0}^{n} \frac{1}{k !(n-k) !} \sum_{w \in \mathfrak{S}_{n}}\left(q_{\{w(1), \ldots, w(k)\}}(C) \cdot \Delta_{\{w(k+1), \ldots, w(n)\}}^{2}\right)
$$

by

$$
\begin{aligned}
& \Delta_{\left\{i_{1}, \ldots, i_{k}\right\}}= \sum_{0 \leq j \leq\left[\frac{k}{2}\right]} \frac{1}{2^{k} j !(k-2 j) !} \\
& \times \sum_{w \in W\left(B_{k}\right)} \varepsilon(w) w\left(u_{e_{i_{1}}-e_{i_{2}}}(x) u_{e_{i_{3}}-e_{i_{4}}}(x) \cdots u_{e_{i_{2 j-1}}-e_{i_{2 j}}}(x) \cdot \partial_{i_{2 j+1}} \partial_{i_{2 j+2}} \cdots \partial_{i_{k}}\right), \\
& q_{\left\{i_{1}, \ldots, i_{k}\right\}}(C)=\sum_{\nu=1}^{k} \sum_{I_{1} \amalg \cdots \amalg I_{\nu}=\left\{i_{1}, \ldots, i_{k}\right\}} T_{I_{1}} \cdots T_{I_{\nu}}, \\
& T_{I}=(-1)^{\# I-1}\left(C S_{I}^{o}-\sum_{j=0}^{3} C_{j} T_{I}^{o}\left(v^{j}\right)\right),
\end{aligned}
$$

where

$$
\begin{aligned}
& S_{\left\{i_{1}, i_{2}, \ldots, i_{k}\right\}}^{o}=\frac{1}{2} \sum_{w \in W\left(B_{k}\right)} w\left(u_{e_{i_{1}}-e_{i_{2}}}(x) u_{e_{i_{2}}-e_{i_{3}}}(x) \cdots u_{e_{i_{k-1}}-e_{i_{k}}}(x)\right), \\
& S_{\varnothing}^{o}=0, S_{\{k\}}^{o}=1, \quad S_{\{i, j\}}^{o}=2 u_{e_{i}-e_{j}}(x)+2 u_{e_{i}+e_{j}}(x), \\
& T_{\varnothing}^{o}\left(v^{j}\right)=0, \quad T_{\{k\}}^{o}\left(v^{j}\right)=2 v_{e_{k}}^{j}(x) \quad \text { for } \quad 1 \leq k \leq n, \\
& q_{\varnothing}=1, \quad q_{\{i\}}=T_{\{i\}}, \quad q_{\left\{i_{1} i_{2}\right\}}=T_{\left\{i_{1}\right\}} T_{\left\{i_{2}\right\}}+T_{\left\{i_{1}, i_{2}\right\}}, \quad \ldots
\end{aligned}
$$

In the above, we identify $W\left(B_{k}\right)$ with the reflection group generated by $w_{e_{i_{k}}}$ and $w_{e_{i_{\nu}}-e_{i_{\nu+1}}}$ $(\nu=1, \ldots, k-1)$. The sum in (5.2) runs over all the partitions of the set $I$ and the order of the subsets $I_{1}, \ldots, I_{\nu}$ is ignored.

Replacing $\partial_{j}$ by $\xi_{j}$ for $j=1, \ldots, n$ in the definition of $\Delta_{\left\{i_{1}, \ldots, i_{k}\right\}}$ and $P_{n}(C)$, we define functions $\bar{\Delta}_{\left\{i_{1}, \ldots, i_{k}\right\}}$ and $\bar{P}_{n}(C)$ of $(x, \xi)$, respectively.

We will define $u_{\alpha}(x)$ and $T_{I}^{o}\left(v^{j}\right)$ so that

$$
\left[P_{n}(C), P_{n}\left(C^{\prime}\right)\right]=0 \quad \text { for } \quad C, C^{\prime} \in \mathbb{C} .
$$

Then putting

$$
\begin{aligned}
& q_{I}^{o}=\left.q_{I}\right|_{C=0}, \\
& P_{n}=P_{n}(0)=\sum_{k=0}^{n} \frac{1}{k !(n-k) !} \sum_{w \in \mathfrak{S}_{n}}\left(q_{\{w(1), \ldots, w(k)\}}^{o} \Delta_{\{w(k+1), \ldots, w(n)\}}^{2}\right), \\
& P_{n-j}=\sum_{i=j}^{n} \sum_{k=i}^{n} \frac{(-1)^{i-j}}{i !(k-i) !(n-k) !} \sum_{w \in \mathfrak{S}_{n} I_{1} \amalg \cdots \amalg I_{j}=w(\{1, \ldots, i\})} \sum_{I_{1} \cdots S_{I_{j}}^{o} q_{w(\{i+1, \ldots, k\})}^{o} \Delta_{w(\{k+1, \ldots, n\})}^{2},} S^{o},
\end{aligned}
$$

we have $P_{n}(C)=\sum_{j=0}^{n} C^{j} P_{n-j}$ and (1.4) and then

$$
\begin{aligned}
& {\left[P_{i}, P_{j}\right]=0 \quad \text { for } \quad 1 \leq i<j \leq n,} \\
& -\frac{P_{1}}{2}=-\frac{1}{2} \sum_{j=1}^{n} \frac{\partial^{2}}{\partial x_{j}^{2}}+\sum_{1 \leq i<j \leq n}\left(u_{e_{i}-e_{j}}(x)+u_{e_{i}+e_{j}}(x)\right)+\sum_{j=0}^{3} C_{j} v^{j}(x) .
\end{aligned}
$$


Remark 9. i) When $n=2, T(x, y)$ in the last section corresponds to $T_{12}$, namely

$$
T\left(x_{1}, x_{2}\right)=\left.T_{12}\right|_{C=0} .
$$

ii) Put

$$
U(x)=\sum_{1 \leq i<j \leq n}\left(u_{i j}^{-}\left(x_{i}-x_{j}\right)+u_{i j}^{+}\left(x_{i}+x_{j}\right)\right) \quad \text { and } \quad V(x)=\sum_{k=1}^{n} v_{k}\left(x_{k}\right)
$$

in (1.3) and let $T_{I}(U ; V)$ be the corresponding $T_{I}$ given by (5.3). Then [29, Remark 4.3] says

$$
T_{I}\left(c_{0} U ; c_{1} V+c_{2} W\right)=c_{0}^{\# I-1} c_{1} T_{I}(U ; V)+c_{0}^{\# I-1} c_{2} T_{I}(U ; W) \quad \text { for } \quad c_{i} \in \mathbb{C} .
$$

iii) The definition (5.3) may be replaced by

$$
T_{I}=(-1)^{\# I-1}\left(C \sum_{\nu=1}^{\# I} \sum_{I_{1} \amalg \cdots \amalg I_{\nu}=I}(-2 \lambda)^{\nu-1}(\nu-1) ! \cdot S_{I_{1}}^{o} \cdots S_{I_{\nu}}^{o}-\sum_{j=0}^{3} C_{j} T_{I}^{o}\left(v^{j}\right)\right)
$$

because $\lambda$ can be any complex number in [29, Lemma 5.2 ii)] when $v=C$. Note that we fixed $\lambda=1$ in [29]. Combining (5.9) and (5.8), we may put

$$
T_{I}=(-1)^{\# I-1}\left(C S_{I}^{o}+c^{\prime} \sum_{\nu \geq 2} c^{\nu-1} \sum_{I_{1} \amalg \cdots \amalg I_{\nu}=I} S_{I_{1}}^{o} \cdots S_{I_{\nu}}^{o}-\sum_{j=0}^{3} C_{j} T_{I}^{o}\left(v^{j}\right)\right)
$$

for any $c, c^{\prime} \in \mathbb{C}$ and hence

$$
T_{I}=(-1)^{\# I-1}\left(C S_{I}^{o}+\sum_{\nu \geq 2} c_{\nu} \sum_{I_{1} \amalg \cdots \amalg I_{\nu}=I} S_{I_{1}}^{o} \cdots S_{I_{\nu}}^{o}-\sum_{j=0}^{3} C_{j} T_{I}^{o}\left(v^{j}\right)\right)
$$

for any $c_{2}, c_{3}, \ldots \in \mathbb{C}$.

Theorem 4 (Ellip- $B_{n},[25],[29$, Theorem 7.2]). Put

$$
\begin{array}{ll}
u_{e_{i} \pm e_{j}}(x)=A \wp_{0}\left(x_{i} \pm x_{j} ; 2 \omega_{1}, 2 \omega_{2}\right) & \text { for } 1 \leq i<j \leq n, \\
v_{e_{k}}^{j}(x)=\wp_{0}\left(x_{k}+\omega_{j} ; 2 \omega_{1}, 2 \omega_{2}\right) & \text { for } 1 \leq k \leq n \text { and } 0 \leq j \leq 3
\end{array}
$$

and

$$
\begin{aligned}
& T_{I}^{O}\left(v^{j}\right)=\sum_{\nu=1}^{\# I} \sum_{I_{1} \amalg \cdots \amalg I_{\nu}=I}(-A)^{\nu-1}(\nu-1) ! \cdot S_{I_{1}}\left(v^{j}\right) \cdots S_{I_{\nu}}\left(v^{j}\right), \\
& S_{\left\{i_{1}, \ldots, i_{k}\right\}}\left(v^{j}\right)=\sum_{w \in W\left(B_{k}\right)} v_{w\left(e_{i_{1}}\right)}^{j}(x) u_{w\left(e_{i_{1}}-e_{i_{2}}\right)}(x) u_{w\left(e_{i_{2}}-e_{i_{3}}\right)}(x) \cdots u_{w\left(e_{i_{k-1}}-e_{i_{k}}\right)}(x) .
\end{aligned}
$$

Then (5.4) holds.

Example 1. Put $v_{k}^{j}=v_{e_{k}}^{j}, \tilde{v}_{k}=\sum_{j=0}^{3} C_{j} v_{k}^{j}$, and $w_{i j}^{ \pm}=u_{i j}^{-} \pm u_{i j}^{+}$. Then

$$
\begin{aligned}
& \Delta_{\{1\}}=\partial_{1}, \\
& \Delta_{\{1,2\}}=\partial_{1} \partial_{2}+u_{12}^{-}-u_{12}^{+}=\partial_{1} \partial_{2}+w_{12}^{-}, \\
& \Delta_{\{1,2,3\}}=\partial_{1} \partial_{2} \partial_{3}+w_{12}^{-} \partial_{3}+w_{23}^{-} \partial_{1}+w_{13}^{-} \partial_{2},
\end{aligned}
$$




$$
\begin{aligned}
& \Delta_{\{1,2,3,4\}}=\partial_{1} \partial_{2} \partial_{3} \partial_{4}+w_{34}^{-} \partial_{1} \partial_{2}+w_{24}^{-} \partial_{1} \partial_{3}+w_{23}^{-} \partial_{1} \partial_{4}+w_{14}^{-} \partial_{2} \partial_{3}+w_{13}^{-} \partial_{2} \partial_{4} \\
& +w_{12}^{-} \partial_{3} \partial_{4}+w_{12}^{-} w_{34}^{-}+w_{13}^{-} w_{24}^{-}+w_{14}^{-} w_{23}^{-}, \\
& S_{1}\left(v^{j}\right)=2 v_{1}^{j} \text {, } \\
& S_{\{1,2\}}\left(v^{j}\right)=2 v_{1}^{j} u_{12}^{-}+2 v_{1}^{j} u_{12}^{+}+2 v_{2}^{j} u_{12}^{-}+2 v_{2}^{j} u_{12}^{+}=2\left(v_{1}^{j}+v_{2}^{j}\right)\left(u_{12}^{-}+u_{12}^{+}\right) \text {, } \\
& S_{\{1,2,3\}}\left(v^{j}\right)=2 v_{1}^{j} u_{12}^{-} u_{23}^{-}+2 v_{1}^{j} u_{12}^{-} u_{23}^{+}+2 v_{1}^{j} u_{12}^{+} u_{23}^{-}+2 v_{1}^{j} u_{12}^{+} u_{23}^{+}+\cdots \\
& =2\left(v_{1}^{j}+v_{3}^{j}\right) w_{12}^{+} w_{23}^{+}+2\left(v_{1}^{j}+v_{2}^{j}\right) w_{23}^{+} w_{13}^{+}+2\left(v_{2}^{j}+v_{3}^{j}\right) w_{12}^{+} w_{13}^{+}, \\
& T_{\{1\}}=C S_{\{1\}}-\sum_{j=0}^{3} C_{j} T_{\{1\}}^{o}\left(v^{j}\right)=C-2 \tilde{v}_{1} \text {, } \\
& T_{\{1,2\}}=-C S_{\{1,2\}}^{o}+\sum_{j=0}^{3} C_{j} T_{\{1,2\}}^{o}\left(v^{j}\right), \\
& q_{\{1\}}=T_{\{1\}}, \\
& q_{\{1,2\}}=T_{\{1\}} T_{\{2\}}+T_{\{1,2\}} \text {, } \\
& q_{\{1,2,3\}}=T_{\{1\}} T_{\{2\}} T_{\{3\}}+T_{\{1,2\}} T_{\{3\}}+T_{\{1,3\}} T_{\{2\}}+T_{\{1\}} T_{\{2,3\}}+T_{\{1,2,3\}} .
\end{aligned}
$$

If $T^{o}\left(v^{j}\right)$ and $S_{i_{1}, \ldots, i_{k}}\left(v^{j}\right)$ are given by (5.10) and (5.11), then

$$
\begin{aligned}
& T_{\{1\}}^{o}\left(v^{j}\right)=2 v_{1}^{j}, \\
& T_{\{1,2\}}^{o}\left(v^{j}\right)=S_{\{1,2\}}\left(v^{j}\right)-A T_{\{1\}}^{o} T_{\{2\}}^{o}=2\left(v_{1}^{j}+v_{2}^{j}\right) w_{12}^{+}-4 A v_{1}^{j} v_{2}^{j}, \\
& T_{\{1,2,3\}}^{o}\left(v^{j}\right)=S_{\{1,2,3\}}\left(v^{j}\right)-2 A\left(v_{1}^{j} S_{\{2,3\}}\left(v^{j}\right)+v_{2}^{j} S_{\{1,3\}}\left(v^{j}\right)+v_{3}^{j} S_{\{1,2\}}\left(v^{j}\right)\right)+16 A^{2} v_{1}^{j} v_{2}^{j} v_{3}^{j} .
\end{aligned}
$$

In particular, if $n=2$, then

$$
\begin{aligned}
P_{2}(C)= & \Delta_{\{1,2\}}^{2}+q_{\{1\}} \Delta_{\{2\}}^{2}+q_{\{2\}} \Delta_{\{1\}}^{2}+q_{\{1,2\}}=\left(\partial_{1} \partial_{2}+u_{12}^{-}-u_{12}^{+}\right)^{2} \\
& +T_{\{1\}} \partial_{2}^{2}+T_{\{2\}} \partial_{1}^{2}+T_{\{1\}} T_{\{2\}}-C S_{\{1,2\}}^{o}+\sum_{j=0}^{3} C_{j} T_{\{1,2\}}^{o}\left(v^{j}\right) \\
= & \left(\partial_{1} \partial_{2}+u_{12}^{-}-u_{12}^{+}\right)^{2}+\left(C-2 \tilde{v}_{1}\right) \partial_{2}^{2}+\left(C-2 \tilde{v}_{2}\right) \partial_{1}^{2}+\left(C-2 \tilde{v}_{1}\right)\left(C-2 \tilde{v}_{2}\right) \\
& -2 C\left(u_{12}^{-}+u_{12}^{+}\right)+2\left(\tilde{v}_{1}+\tilde{v}_{2}\right)\left(u_{12}^{-}+u_{12}^{+}\right)-4 A \sum_{j=0}^{3} C_{j} v_{1}^{j} v_{2}^{j}=C^{2}-2 P \cdot C+P_{2}
\end{aligned}
$$

with

$$
\begin{aligned}
& P=-\frac{1}{2}\left(\partial_{1}^{2}+\partial_{2}^{2}\right)+\tilde{v}_{1}+\tilde{v}_{2}+u_{12}^{-}+u_{12}^{+}, \\
& P_{2}=\left(\partial_{1} \partial_{2}+u_{12}^{-}-u_{12}^{+}\right)^{2}-2 \tilde{v}_{1} \partial_{2}^{2}-2 \tilde{v}_{2} \partial_{1}^{2}+4 \tilde{v}_{1} \tilde{v}_{2}+2\left(\tilde{v}_{1}+\tilde{v}_{2}\right)\left(u_{12}^{-}+u_{12}^{+}\right)-4 A \sum_{j=0}^{3} C_{j} v_{1}^{j} v_{2}^{j},
\end{aligned}
$$

which should be compared with (4.2), (4.4) and (4.6).

In general

$$
\begin{aligned}
& P_{1}=\sum_{k=1}^{n}\left(\Delta_{\{k\}}^{2}+q_{\{k\}}^{o}\right)-\sum_{1 \leq i<j \leq n} S_{\{i, j\}}^{o}=\sum_{k=1}^{n}\left(\partial_{k}^{2}-2 \tilde{v}_{k}^{j}\right)-2 \sum_{1 \leq i<j \leq n} w_{i j}^{+}, \\
& P_{2}=\sum_{1 \leq i<j \leq n} \Delta_{\{i, j\}}^{2}+\sum_{\substack{1 \leq i \leq n \\
1 \leq j \leq n, i \neq j}} \sum_{j=1}^{n} q_{\{i\}}^{o} \Delta_{\{j\}}^{2}+\sum_{1 \leq i<j \leq n} q_{\{i, j\}}^{o},
\end{aligned}
$$




$$
\begin{aligned}
& -\sum_{\substack{1 \leq i<j \leq n \\
1 \leq k \leq n, k \neq i, j}} S_{\{i, j\}}^{o}\left(\Delta_{\{k\}}^{2}+q_{\{k\}}^{o}\right)+\sum_{\substack{1 \leq i<j \leq n \\
i<k<\ell \leq n \\
j \neq k, \ell}} S_{\{i, j\}}^{o} S_{\{k, \ell\}}^{o}+\sum_{1 \leq i<j<k \leq n} S_{\{i, j, k\}}^{o} \\
= & \sum\left(\partial_{i} \partial_{j}+w_{i j}^{-}\right)^{2}-\sum 2 \tilde{v}_{i} \partial_{j}^{2}+\sum 4 \tilde{v}_{i} \tilde{v}_{j}+\sum C_{j} T_{\{k, \ell\}}^{o}\left(v^{j}\right) \\
& -2 \sum w_{i j}^{+}\left(\partial_{k}^{2}-2 \tilde{v}_{k}\right)+4 \sum w_{i j}^{+} w_{k \ell}^{+}+2 \sum w_{i j}^{+} w_{j k}^{+} .
\end{aligned}
$$

Here if (5.10) and (5.11) are valid, then

$$
T_{\{k, \ell\}}^{o}\left(v^{j}\right)=2\left(v_{k}^{j}+v_{\ell}^{j}\right) w_{k \ell}^{+}-4 A v_{k}^{j} v_{\ell}^{j} .
$$

The commuting operator $P_{3}$ of the 6 -th order is

$$
\begin{aligned}
P_{3}= & \sum \Delta_{\{i, j, k\}}^{2}+\sum q_{\{k\}}^{o} \Delta_{\{i, j\}}^{2}+\sum q_{\{i, j, k\}}^{o}-\sum S_{\{k, \ell\}}^{o} \Delta_{\{i, j\}}^{2} \\
& -\sum S_{\{k, \ell\}}^{o} q_{\{i\}}^{o} \Delta_{\{j\}}^{2}-\sum S_{\{k, \ell\}}^{o} q_{\{i, j\}}^{o}+\sum S_{\{i, j, k, \ell, m\}}^{o}+\sum S_{\{i, j, k, \ell\}}^{o} S_{\{\mu, \nu\}}^{o} \\
& +\sum S_{\{i, j, k\}}^{o} S_{\{\ell, \mu, \nu\}}^{o}+\sum S_{\{i, j, k\}}^{o} S_{\{\ell, m\}}^{o} S_{\{\mu, \nu\}}^{o}+\sum S_{\left\{i_{1}, i_{2}\right\}}^{o} S_{\left\{j_{1}, j_{2}\right\}}^{o} S_{\left\{k_{1}, k_{2}\right\}}^{o} S_{\left\{\ell_{1}, \ell_{2}\right\}}^{o} .
\end{aligned}
$$

In Theorem 4 the Schrödinger operator is

$$
P=-\frac{1}{2} \sum_{k=0}^{n} \frac{\partial^{2}}{\partial x_{k}^{2}}+A \sum_{1 \leq i<j \leq n}\left(\wp\left(x_{i}-x_{j}\right)+\wp\left(x_{i}+x_{j}\right)\right)+\sum_{j=0}^{3} C_{j} \sum_{k=1}^{n} \wp\left(x_{k}+\omega_{j}\right)
$$

and the operator $P_{2}$ satisfying $\left[P, P_{2}\right]=0$ is given by (5.12) and (5.13) with

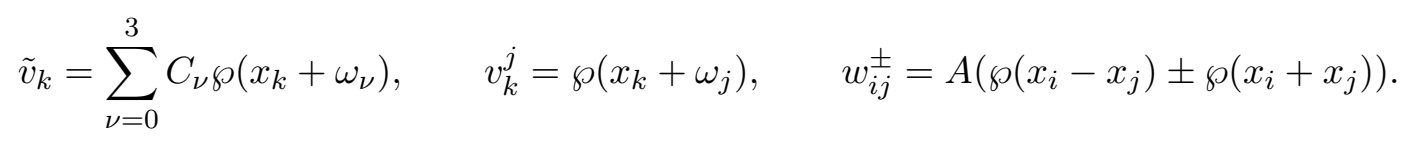

\subsection{Analytic continuation of integrals}

\section{Theorem 5 (Toda- $D_{n}^{(1)}$-bry). For}

$$
\begin{aligned}
& u_{e_{i}-e_{j}}(x)= \begin{cases}A e^{-2 \lambda\left(x_{i}-x_{i+1}\right)} & (j=i+1), \\
0 & (|j-i|>1),\end{cases} \\
& u_{e_{i}+e_{j}}(x)= \begin{cases}A e^{2 \lambda\left(x_{1}+x_{2}\right)} & (i+j=3), \\
A e^{-2 \lambda\left(x_{n-1}+x_{n}\right)} & (i+j=2 n-1), \\
0 & (i+j \notin\{3,2 n-1\}),\end{cases} \\
& v_{k}^{0}(x)=\delta_{1 k} \sinh ^{-2} \lambda x_{1}, \quad v_{k}^{1}(x)=\delta_{1 k} \sinh ^{-2} 2 \lambda x_{1}, \\
& v_{k}^{2}(x)=\delta_{n k} \sinh ^{-2} \lambda x_{n}, \quad v_{k}^{3}(x)=\delta_{n k} \sinh ^{-2} 2 \lambda x_{n},
\end{aligned}
$$

we have commuting integrals $P_{j}$ by (5.5), (5.1), (5.2), (5.3) and

$$
\begin{aligned}
& S_{\{k\}}^{o}=1 \quad \text { for } \quad 1 \leq k \leq n, \\
& S_{I}^{o}=0 \quad \text { if } \quad I \neq\{k, k+1, \ldots, \ell\} \quad \text { for } \quad 1 \leq k<\ell \leq n, \\
& S_{\{k, k+1, \ldots, \ell\}}^{o}=2 A^{\ell-k+1}\left(e^{-2 \lambda\left(x_{k}-x_{\ell}\right)}+\delta_{1 k} e^{2 \lambda\left(x_{1}+x_{\ell}\right)}+\delta_{\ell n} e^{-2 \lambda\left(x_{k}+x_{n}\right)}\right) \text {, } \\
& T_{\{k\}}^{0}\left(v^{j}\right)=2 v_{k}^{j}(x) \quad \text { for } \quad 0 \leq j \leq 3, \quad k=1, \ldots, n, \\
& T_{I}^{0}\left(v^{0}\right)=0 \quad \text { if } \quad I \neq\{1, \ldots, k\} \quad \text { for } \quad k=1, \ldots, n \text {, } \\
& T_{I}^{0}\left(v^{2}\right)=0 \quad \text { if } \quad I \neq\{k, \ldots, n\} \quad \text { for } \quad k=1, \ldots, n,
\end{aligned}
$$




$$
\begin{aligned}
& T_{I}^{0}\left(v^{1}\right)=T_{I}^{0}\left(v^{3}\right)=0 \quad \text { if } \quad \# I>1, \\
& T_{\{1, \ldots, k\}}^{0}\left(v^{0}\right)=8 A^{k-1}\left(e^{2 \lambda x_{k}}+\delta_{k n} e^{-2 \lambda x_{n}}\right) \quad \text { for } \quad k \geq 2, \\
& T_{\{n-k+1, \ldots, n\}}^{0}\left(v^{2}\right)=8 A^{k-1}\left(e^{-2 \lambda x_{n-k+1}}+\delta_{k n} e^{2 \lambda x_{1}}\right) \quad \text { for } \quad k \geq 2 .
\end{aligned}
$$

Proof. Put

$$
\begin{aligned}
& \tilde{x}=\left(x_{1}-\frac{1-1}{n-1} \omega_{2}, \ldots, x_{k}-\frac{k-1}{n-1} \omega_{2}, \ldots, x_{n}-\frac{n-1}{n-1} \omega_{2}\right), \\
& \tilde{u}_{e_{i} \mp e_{j}}(\tilde{x})=A \frac{e^{2 \lambda \omega_{2} /(n-1)}}{4 \lambda^{2}} \wp_{0}\left(x_{i}-\frac{i-1}{n-1} \omega_{2} \mp\left(x_{j}-\frac{j-1}{n-1} \omega_{2}\right) ; 2 \omega_{1}, 2 \omega_{2}\right), \\
& \tilde{v}_{k}^{j}(\tilde{x})=\frac{(-1)^{j}}{\lambda^{2}} \wp_{0}\left(x_{k}-\frac{k-1}{n-1} \omega_{2}+\omega_{j} ; 2 \omega_{1}, 2 \omega_{2}\right) \quad \text { for } \quad 0 \leq j \leq 3, \quad 1 \leq k \leq n .
\end{aligned}
$$

When $\omega_{2} \rightarrow \infty, \tilde{u}_{e_{i} \mp e_{j}}(\tilde{x})$ and $\tilde{v}_{k}^{\ell}(\ell=0,1,2,3)$ converge to $u_{e_{i} \mp e_{j}}(x)$ in $(5.14)$ and

$$
\begin{aligned}
& v_{k}^{0}(x)=\delta_{1 k} \sinh ^{-2} \lambda x_{1}, \quad v_{k}^{1}(x)=\delta_{1 k} \cosh ^{-2} \lambda x_{1}, \\
& v_{k}^{2}(x)=\delta_{n k} \sinh ^{-2} \lambda x_{n}, \quad v_{k}^{3}(x)=\delta_{n k} \cosh ^{-2} \lambda x_{n},
\end{aligned}
$$

respectively. Under the notation in Theorem 4 , let $\tilde{S}_{I}\left(\tilde{v}^{\ell}\right)$ and $\tilde{T}_{I}^{o}\left(\tilde{v}^{\ell}\right)$ be the functions defined in the same way as $S_{I}\left(v^{\ell}\right)$ and $T_{I}^{o}\left(v^{\ell}\right)$, respectively, where $\left(u_{e_{i} \mp e_{j}}(x), v_{k}^{\ell}(x)\right)$ are replaced by $\left(\tilde{u}_{e_{i} \mp e_{j}}(x), \tilde{v}_{k}^{\ell}(\tilde{x})\right)$. Then by taking the limits for $\omega_{2} \rightarrow \infty, \tilde{T}_{I}^{o}\left(\tilde{v}^{\ell}\right)$ converge to the following $\bar{T}_{I}^{o}\left(v^{\ell}\right)$

$$
\begin{array}{lllll}
\bar{T}_{I}^{0}\left(v^{0}\right)=\bar{T}_{I}^{0}\left(v^{1}\right)=0 & \text { if } & I \neq\{1, \ldots, k\} & \text { for } & k=1, \ldots, n, \\
\bar{T}_{I}^{0}\left(v^{2}\right)=\bar{T}_{I}^{0}\left(v^{3}\right)=0 & \text { if } & I \neq\{k, \ldots, n\} & \text { for } & k=1, \ldots, n .
\end{array}
$$

If $k \geq 2$, then

$$
\begin{aligned}
& \bar{T}_{\{1, \ldots, k\}}^{0}\left(v^{0}\right)= \lim _{\omega_{2} \rightarrow \infty} \sum_{\nu=1}^{\# I} \sum_{I_{1} \amalg \cdots \amalg I_{\nu}=I}\left(-A \frac{e^{2 \lambda \omega_{2} /(n-1)}}{4 \lambda^{2}}\right)^{\nu-1}(\nu-1) ! \tilde{S}_{I_{1}}\left(\tilde{v}^{0}\right) \cdots \tilde{S}_{I_{\nu}}\left(\tilde{v}^{0}\right) \\
&= \lim _{\omega_{2} \rightarrow \infty} \tilde{S}_{\{1, \ldots, k\}}\left(\tilde{v}^{0}\right)-\lim _{\omega_{2} \rightarrow \infty} \tilde{S}_{\{1\}}\left(\tilde{v}^{0}\right) A \frac{e^{2 \lambda \omega_{2} /(n-1)}}{4 \lambda^{2}} \tilde{S}_{\{2, \ldots, k\}}\left(\tilde{v}^{0}\right) \\
&= 2 A^{k-1} \sinh ^{-2} \lambda x_{1}\left(e^{-2 \lambda\left(x_{1}-x_{k}\right)}+e^{2 \lambda\left(x_{1}+x_{k}\right)}+\delta_{k n} e^{2 \lambda\left(x_{1}-x_{n}\right)}+\delta_{k n} e^{-2 \lambda\left(x_{1}+x_{n}\right)}\right) \\
&-2 \sinh ^{-2} \lambda x_{1} \cdot 2 A^{k-1}\left(e^{2 \lambda x_{k}}+\delta_{k n} e^{-2 \lambda x_{n}}\right)=8 A^{k-1}\left(e^{2 \lambda x_{k}}+\delta_{k n} e^{-2 \lambda x_{n}}\right), \\
& \bar{T}_{\{1, \ldots, k\}}^{0}\left(v^{1}\right)= 2 A^{k-1} \cosh ^{-2} \lambda x_{1}\left(e^{-2 \lambda\left(x_{1}-x_{k}\right)}+e^{2 \lambda\left(x_{1}+x_{k}\right)}+\delta_{k n} e^{2 \lambda\left(x_{1}-x_{n}\right)}+\delta_{k n} e^{-2 \lambda\left(x_{1}+x_{n}\right)}\right) \\
& \quad+4 A^{k-1} \cosh ^{-2} \lambda x_{1}\left(e^{2 \lambda x_{k}}+\delta_{k n} e^{-2 \lambda x_{n}}\right)=8 A^{k-1}\left(e^{2 \lambda x_{k}}+\delta_{k n} e^{-2 \lambda x_{n}}\right), \\
& \bar{T}_{\{n-k+1, \ldots, n\}}^{0}\left(v^{2}\right)=8 A^{k-1}\left(e^{-2 \lambda x_{n-k+1}}+\delta_{k n} e^{2 \lambda x_{1}}\right), \\
& \bar{T}_{\{n-k+1, \ldots, n\}}^{0}\left(v^{3}\right)=8 A^{k-1}\left(e^{-2 \lambda x_{n-k+1}}+\delta_{k n} e^{2 \lambda x_{1}}\right) .
\end{aligned}
$$

Replacing $v^{1}$ and $v^{3}$ by $(1 / 4)\left(v^{0}-v^{1}\right)$ and $(1 / 4)\left(v^{2}-v^{3}\right)$, respectively, we have the theorem by the analytic continuation given in Lemma 2.

As is proved by [29], suitable limits of the functions in Theorem 4 give the following theorem.

Theorem 6 (Trig- $\boldsymbol{B}_{\boldsymbol{n}},\left[29\right.$, Proposition 6.1]). For complex numbers $\lambda, C, C_{0}, \ldots, C_{3}$ and $A$ with $\lambda \neq 0$, we have (5.7) by putting

$$
u_{e_{i} \pm e_{j}}(x)=A \sinh ^{-2} \lambda\left(x_{i} \pm x_{k}\right),
$$




$$
\begin{aligned}
& v_{e_{k}}^{0}(x)=\sinh ^{-2} \lambda x_{k}, \\
& v_{e_{k}}^{2}(x)=\sinh ^{2} \lambda x_{k}, \quad v_{e_{k}}^{1}(x)=\cosh ^{-2} \lambda x_{k},
\end{aligned}
$$

and

$$
\begin{aligned}
& T_{I}=(-1)^{\# I-1}\left(C S_{I}^{o}-C_{0} T_{I}^{o}\left(v^{0}\right)-C_{1} T_{I}^{o}\left(v^{1}\right)-C_{2} S_{I}\left(v^{2}\right)-C_{3} S_{I}\left(v^{3}\right)\right. \\
& \left.\quad+2 C_{3} \sum_{I_{1} \amalg I_{2}=I}\left(S_{I_{1}}\left(v^{2}\right) S_{I_{2}}\left(v^{2}\right)+S_{I_{1}}\left(v^{2}\right) S_{I_{2}}^{o}+S_{I_{1}}^{o} S_{I_{2}}\left(v^{2}\right)\right)\right), \\
& T_{I}^{o}\left(v^{0}\right)=\sum_{\nu=1}^{\# I} \sum_{I_{1} \amalg \cdots \amalg I_{\nu}=I}(-A)^{\nu-1}(\nu-1) ! S_{I_{1}}\left(v^{0}\right) \cdots S_{I_{\nu}}\left(v^{0}\right), \\
& T_{I}^{o}\left(v^{1}\right)=\sum_{\nu=1}^{\# I} \sum_{I_{1} \amalg \cdots \amalg I_{\nu}=I} A^{\nu-1}(\nu-1) ! S_{I_{1}}\left(v^{1}\right) \cdots S_{I_{\nu}}\left(v^{1}\right) .
\end{aligned}
$$

\section{Theorem 7 (Trig- $\boldsymbol{A}_{\boldsymbol{n}-\mathbf{1}}$-bry). For}

$$
\begin{aligned}
& u_{e_{i}-e_{j}}(x)=A \sinh ^{-2} \lambda\left(x_{i}-x_{j}\right), \quad u_{e_{i}+e_{j}}(x)=0, \\
& v_{e_{k}}^{0}(x)=e^{-2 \lambda x_{k}}, \quad v_{e_{k}}^{1}(x)=e^{-4 \lambda x_{k}}, \quad v_{e_{k}}^{2}(x)=e^{2 \lambda x_{k}}, \quad v_{e_{k}}^{3}(x)=e^{4 \lambda x_{k}}
\end{aligned}
$$

we have (5.7) by putting

$$
T_{I}=(-1)^{\# I-1}\left(C S_{I}^{o}-\sum_{j=0}^{3} C_{j} S_{I}\left(v^{j}\right)+2 \sum_{I_{1} \amalg I_{2}=I}\left(C_{1} S_{I_{1}}\left(v^{0}\right) S_{I_{2}}\left(v^{0}\right)+C_{3} S_{I_{1}}\left(v^{2}\right) S_{I_{2}}\left(v^{2}\right)\right)\right) .
$$

Proof. Putting

$$
\begin{aligned}
& \tilde{u}_{e_{i} \pm e_{j}}=A \sinh ^{-2} \lambda\left(\left(x_{i}+N\right) \pm\left(x_{j}+N\right)\right), \quad \tilde{v}_{k}^{0}=\frac{1}{4} e^{2 \lambda N} \sinh ^{-2} \lambda\left(x_{k}+N\right), \\
& \tilde{v}_{k}^{1}=\frac{1}{4} e^{4 \lambda N} \sinh ^{-2} 2 \lambda\left(x_{k}+N\right)=\frac{1}{16} e^{4 \lambda N}\left(\sinh ^{-2} 2 \lambda\left(x_{k}+N\right)-\cosh ^{-2} 2 \lambda\left(x_{k}+N\right)\right), \\
& \tilde{v}_{k}^{2}=4 e^{-2 \lambda N} \sinh ^{2} \lambda\left(x_{k}+N\right), \quad \tilde{v}_{k}^{3}=4 e^{-4 \lambda N} \sinh ^{2} 2 \lambda\left(x_{k}+N\right), \\
& \tilde{x}=\left(x_{1}+N, x_{2}+N, \ldots, x_{n}+N\right)
\end{aligned}
$$

under the notation in Theorem 6, we have

$$
\begin{aligned}
& \left(\bar{u}_{e_{i}-e_{j}}, \bar{u}_{e_{i}+e_{j}}, \bar{v}_{k}^{0}, \bar{v}_{k}^{1}, \bar{v}_{k}^{2}, \bar{v}_{k}^{3}\right):=\lim _{N \rightarrow \infty}\left(\tilde{u}_{e_{i}-e_{j}}, \tilde{u}_{e_{i}+e_{j}}, \tilde{v}_{k}^{0}, \tilde{v}_{k}^{1}, \tilde{v}_{k}^{2}, \tilde{v}_{k}^{3}\right) \\
& \quad=\left(A \sinh ^{-2} \lambda\left(x_{i}-x_{k}\right), 0, e^{-2 \lambda x_{k}}, e^{-4 \lambda x_{k}} e^{2 \lambda x_{k}}, e^{4 \lambda x_{k}}\right), \\
& \lim _{N \rightarrow \infty} \frac{1}{4} e^{2 \lambda N} T_{I}^{o}\left(v^{0}\right)(\tilde{x})=\bar{S}_{I}\left(\bar{v}^{0}\right), \\
& \lim _{N \rightarrow \infty} \frac{1}{16} e^{4 \lambda N}\left(T_{I}^{o}\left(v^{0}\right)(\tilde{x})-T_{I}^{o}\left(v^{1}\right)(\tilde{x})\right)=\bar{S}_{I}\left(\bar{v}^{1}\right)-2 \sum_{I_{1} \amalg I_{2}=I} \bar{S}_{I_{1}}\left(\bar{v}^{0}\right) \bar{S}_{I_{2}}\left(\bar{v}^{0}\right), \\
& \lim _{N \rightarrow \infty} 4 e^{-2 \lambda N} T_{I}^{o}\left(v^{2}\right)(\tilde{x})=\bar{S}_{I}\left(\bar{v}^{2}\right), \\
& \lim _{N \rightarrow \infty} 4 e^{-2 \lambda N} T_{I}^{o}\left(v^{3}\right)(\tilde{x})=\bar{S}_{I}\left(\bar{v}^{3}\right)-2 \sum_{I_{1} \amalg I_{2}=I} \bar{S}_{I_{1}}\left(\bar{v}^{2}\right) \bar{S}_{I_{2}}\left(\bar{v}^{2}\right) .
\end{aligned}
$$

Here $\bar{S}_{I}\left(\bar{v}^{\ell}\right)$ are defined by (5.11) with $u_{e_{i} \pm e_{j}}$ and $v_{e_{k}}^{\ell}$ replaced by $\bar{u}_{e_{i} \pm e_{j}}$ and $\bar{v}_{e_{k}}^{\ell}$, respectively. Then the theorem is clear. 
Theorem 8 (Toda- $\boldsymbol{B}_{\boldsymbol{n}}^{(\mathbf{1})}$-bry). For the potential function defined by

$$
\begin{aligned}
& u_{e_{i}-e_{j}}(x)=\left\{\begin{array}{lll}
A e^{-2 \lambda\left(x_{i}-x_{i+1}\right)} & \text { if } & j=i+1, \\
0 & \text { if } & 1 \leq i<i+1<j \leq n,
\end{array}\right. \\
& u_{e_{i}+e_{j}}(x)=\left\{\begin{array}{lll}
A e^{-2 \lambda\left(x_{n-1}+x_{n}\right)} & \text { if } & i=n-1, \quad j=n, \\
0 & \text { if } & 1 \leq i<j \leq n
\end{array} \text { and } \quad i \neq n-1,\right. \\
& v_{k}^{0}(x)=\delta_{k 1} e^{2 \lambda x_{1}}, \quad v_{k}^{1}(x)=\delta_{k 1} e^{4 \lambda x_{1}}, \\
& v_{k}^{2}(x)=\delta_{k n} \sinh ^{-2} \lambda x_{n}, \quad v_{k}^{3}(x)=\delta_{k n} \sinh ^{-2} 2 \lambda x_{n},
\end{aligned}
$$

we have (5.7) with

$$
\begin{aligned}
& S_{\{k\}}^{o}=1 \quad \text { for } \quad 1 \leq k \leq n, \\
& S_{I}^{o}=0 \quad \text { if } \quad I \neq\{k, k+1, \ldots, \ell\} \quad \text { for } \quad 1 \leq k<\ell \leq n, \\
& S_{\{k, k+1, \ldots, \ell\}}^{o}=2 A^{\ell-k+1}\left(e^{-2 \lambda\left(x_{k}-x_{\ell}\right)}+\delta_{\ell n} e^{-2 \lambda\left(x_{k}+x_{n}\right)}\right), \\
& T_{\{k\}}^{0}\left(v^{j}\right)=2 v_{k}^{j}(x) \quad \text { for } \quad 0 \leq j \leq 3, \quad k=1, \ldots, n, \\
& T_{I}^{0}\left(v^{0}\right)=0 \quad \text { if } \quad I \neq\{1, \ldots, k\} \quad \text { for } \quad k=1, \ldots, n, \\
& T_{I}^{0}\left(v^{2}\right)=0 \quad \text { if } \quad I \neq\{k, \ldots, n\} \quad \text { for } \quad k=1, \ldots, n, \\
& T_{I}^{0}\left(v^{1}\right)=T_{I}^{0}\left(v^{3}\right)=0 \quad \text { if } \quad \# I>1, \\
& T_{\{1, \ldots, k\}}^{0}\left(v^{0}\right)=2 A^{k-1}\left(e^{2 \lambda x_{k}}+\delta_{k n} e^{-2 \lambda x_{n}}\right) \quad \text { for } \quad k \geq 2, \\
& T_{\{n-k+1, \ldots, n\}}^{0}\left(v^{2}\right)=8 A^{k-1} e^{-2 \lambda x_{n-k+1}} \quad \text { for } \quad k \geq 2 .
\end{aligned}
$$

Proof. Suppose Re $\lambda>0$. In (Toda- $D_{n}^{(1)}$-bry) put

$$
\begin{aligned}
& \tilde{x}=\left(x_{1}-(n-1) N, \ldots, x_{k}-(n-k) N, \ldots, x_{n}-(n-n) N\right), \\
& \tilde{u}_{e_{i}-e_{j}}= \begin{cases}A e^{-2 \lambda N} e^{-2 \lambda\left(x_{i}-(n-i) N-x_{i+1}+(n-i-1) N\right)} & (j=i+1), \\
0 & (|j-i|>1),\end{cases} \\
& \tilde{u}_{e_{i}+e_{j}}= \begin{cases}A e^{-2 \lambda N} e^{2 \lambda\left(x_{1}-(n-1) N+x_{2}-(n-2) N\right)} & (i+j=3), \\
A e^{-2 \lambda N} e^{-2 \lambda\left(x_{n-1}-N+x_{n}\right)} & (i+j=2 n-1), \\
0 & (i+j \notin\{3,2 n-1\}),\end{cases} \\
& \tilde{v}_{k}^{0}=\delta_{1 k} \frac{e^{2 \lambda(n-1) N}}{4} \sinh ^{-2} \lambda\left(x_{1}-(n-1) N\right), \\
& \tilde{v}_{k}^{1}=\delta_{1 k} \frac{e^{4 \lambda(n-1) N}}{4} \sinh ^{-2} 2 \lambda\left(x_{1}-(n-1) N\right), \\
& \tilde{v}_{k}^{2}=\delta_{n k} \sinh ^{-2} \lambda x_{n}, \quad \tilde{v}_{k}^{3}=\delta_{n k} \sinh ^{-2} 2 \lambda x_{n}
\end{aligned}
$$

and we have (5.15) by the limit $N \rightarrow \infty$. Moreover for $k \geq 2$, it follows from Theorem 5 and (5.8) that

$$
\begin{aligned}
& \tilde{T}_{\{1, \ldots, k\}}\left(\tilde{v}^{1}\right)=\tilde{T}_{\{1, \ldots, k\}}\left(\tilde{v}^{3}\right)=0, \\
& \tilde{T}_{\{1, \ldots, k\}}\left(\tilde{v}^{0}\right)=\frac{1}{4} e^{2 \lambda(n-1) N}\left(A e^{-2 \lambda N}\right)^{k-1}\left(8 e^{2 \lambda\left(x_{k}-(n-k) N\right)}+8 \delta_{k n} e^{-2 \lambda x_{n}}\right) \\
& =2 A^{k-1}\left(e^{2 \lambda x_{k}}+\delta_{k n} e^{-2 \lambda x_{n}}\right), \\
& \tilde{T}_{\{n-k+1, \ldots, n\}}\left(\tilde{v}^{2}\right)=\left(A e^{-2 \lambda N}\right)^{k-1}\left(8 e^{-2 \lambda\left(x_{n-k+1}-(k-1) N\right)}+8 \delta_{1 k} e^{2 \lambda\left(x_{1}-(n-1) N\right)}\right) \\
& =8 A^{k-1} e^{-2 \lambda x_{n-k+1}},
\end{aligned}
$$

which implies the theorem. 
Theorem 9 (Toda- $\left.C_{\boldsymbol{n}}^{(1)}\right)$. For the potential function defined by

$$
\begin{aligned}
& u_{e_{i}-e_{j}}(x)= \begin{cases}A e^{-2 \lambda\left(x_{i}-x_{i+1}\right)} & \text { if } \quad j=i+1, \\
0 & \text { if } \quad 1 \leq i<i+1<j \leq n,\end{cases} \\
& u_{e_{i}+e_{j}}(x)=0 \quad \text { for } \quad 1 \leq i<j \leq n, \\
& v_{k}^{0}(x)=\delta_{k 1} e^{2 \lambda x_{1}}, \quad v_{k}^{1}(x)=\delta_{k 1} e^{4 \lambda x_{1}}, \\
& v_{k}^{2}(x)=\delta_{k n} e^{-2 \lambda x_{n}}, \quad v_{k}^{3}(x)=\delta_{k n} e^{-4 \lambda x_{n}},
\end{aligned}
$$

we have (5.7) with

$$
\begin{aligned}
& S_{\{k\}}^{o}=1 \quad \text { for } \quad 1 \leq k \leq n, \\
& S_{I}^{o}=0 \quad \text { if } \quad I \neq\{k, k+1, \ldots, \ell\} \quad \text { for } \quad 1 \leq k<\ell \leq n \text {, } \\
& S_{\{k, k+1, \ldots, \ell\}}^{o}=2 A^{\ell-k+1} e^{-2 \lambda\left(x_{k}-x_{\ell}\right)} \text {, } \\
& T_{\{k\}}^{0}\left(v^{j}\right)=2 v_{k}^{j}(x) \quad \text { for } \quad 0 \leq j \leq 3, \quad k=1, \ldots, n, \\
& T_{I}^{0}\left(v^{0}\right)=0 \quad \text { if } \quad I \neq\{1, \ldots, k\} \quad \text { for } \quad k=1, \ldots, n \text {, } \\
& T_{I}^{0}\left(v^{2}\right)=0 \quad \text { if } \quad I \neq\{k, \ldots, n\} \quad \text { for } \quad k=1, \ldots, n \text {, } \\
& T_{I}^{0}\left(v^{1}\right)=T_{I}^{0}\left(v^{3}\right)=0 \quad \text { if } \quad \# I>1 \text {, } \\
& T_{\{1, \ldots, k\}}^{0}\left(v^{0}\right)=2 A^{k-1} e^{2 \lambda x_{k}} \quad \text { for } \quad k \geq 2 \text {, } \\
& T_{\{n-k+1, \ldots, n\}}^{0}\left(v^{2}\right)=2 A^{k-1} e^{-2 \lambda x_{n-k+1}} \quad \text { for } \quad k \geq 2 \text {. }
\end{aligned}
$$

Proof. Substituting $x_{k}$ by $x_{k}+R$ for $k=1, \ldots, n$ and multiplying $v_{k}^{0}, v_{k}^{1}, v_{k}^{2}$ and $v_{k}^{3}$ by $e^{-2 \lambda R}$, $e^{-4 \lambda R},(1 / 4) e^{2 \lambda R}$ and $(1 / 4) e^{4 \lambda R}$, respectively, we have the claim from Theorem 8 .

Theorem 10 (Rat- $\boldsymbol{A}_{\boldsymbol{n}-\mathbf{1}}$-bry). We have (5.7) if

$$
\begin{aligned}
& u_{e_{i}-e_{j}}(x)=\frac{A}{\left(x_{i}-x_{j}\right)^{2}}, \quad u_{e_{i}+e_{j}}(x)=0, \quad v_{k}^{j}(x)=x_{k}^{j+1}, \\
& T_{I}=(-1)^{\# I-1}\left(C S_{I}^{o}-\sum_{j=0}^{3} C_{j} S_{I}\left(v^{j}\right)+\sum_{I_{1} \amalg I_{2}=I} C_{1}\left(S_{I_{1}}\left(v^{0}\right) S_{I_{2}}^{o}+S_{I_{1}}^{o} S_{I_{1}}\left(v^{0}\right)\right)\right. \\
& \left.\quad+\sum_{I_{1} \amalg I_{2}=I} C_{3}\left(S_{I_{1}}\left(v^{1}\right) S_{I_{2}}^{o}+S_{I_{1}}\left(v^{0}\right) S_{I_{2}}\left(v^{0}\right)+S_{I_{1}}^{o} S_{I_{2}}\left(v^{1}\right)\right)\right) .
\end{aligned}
$$

Proof. Put

$$
\begin{aligned}
& \tilde{u}_{e_{i}-e_{j}}=\lambda^{2} \sinh ^{-2} \lambda\left(x_{i}-x_{j}\right), \quad \tilde{u}_{e_{i}+e_{j}}=0, \\
& \tilde{v}_{k}^{0}=\frac{1}{2 \lambda}\left(e^{2 \lambda x_{k}}-1\right), \quad \tilde{v}_{k}^{1}=\frac{1}{4 \lambda^{2}}\left(e^{2 \lambda x_{k}}+e^{-2 \lambda x_{k}}-2\right), \\
& \tilde{v}_{k}^{2}=\frac{1}{8 \lambda^{3}}\left(e^{4 \lambda x_{k}}-3 e^{2 \lambda x_{k}}-e^{-2 \lambda x_{k}}+3\right), \quad \tilde{v}_{k}^{3}=\frac{1}{16 \lambda^{4}}\left(e^{4 \lambda x_{k}}+e^{-4 \lambda x_{k}}-4 e^{2 \lambda x_{k}}-4 e^{-2 \lambda x_{k}}+6\right) .
\end{aligned}
$$

Then taking $\lambda \rightarrow 0$ we have the required potential function.

Owing to (Trig- $A_{n-1}$-bry) and Remark 9 , we have

$$
\begin{aligned}
& \lim _{\lambda \rightarrow 0} \tilde{S}_{I}\left(\sum \tilde{v}_{k}^{j}\right)=\bar{S}_{I}\left(\sum x_{k}^{j+1}\right), \\
& \lim _{\lambda \rightarrow 0} \lambda^{2} \frac{1}{8 \lambda^{3}}\left(\tilde{S}_{I_{1}}\left(\sum e^{2 \lambda x_{k}}\right) \tilde{S}_{I_{2}}\left(\sum e^{2 \lambda x_{k}}\right)-4 \tilde{S}_{I_{1}}^{o} \tilde{S}_{I_{2}}^{o}\right)
\end{aligned}
$$




$$
\begin{aligned}
& =\frac{1}{2}\left(\bar{S}_{I_{1}}\left(\sum x_{k}\right) \bar{S}_{I_{2}}^{o}+\bar{S}_{I_{1}}^{o} \bar{S}_{I_{2}}\left(\sum x_{k}\right)\right) \\
\lim _{\lambda \rightarrow 0} \lambda^{2} & \frac{1}{16 \lambda^{4}}\left(\tilde{S}_{I_{1}}\left(\sum e^{2 \lambda x_{k}}\right) \tilde{S}_{I_{2}}\left(\sum e^{2 \lambda x_{k}}\right)+\tilde{S}_{I_{1}}\left(\sum e^{-2 \lambda x_{k}}\right) \tilde{S}_{I_{2}}\left(\sum e^{-2 \lambda x_{k}}\right)-8 \tilde{S}_{I_{1}}^{o} \tilde{S}_{I_{2}}^{o}\right) \\
& =\frac{1}{2}\left(\bar{S}_{I_{1}}\left(\sum x_{k}^{2}\right) \bar{S}_{I_{2}}^{o}+\bar{S}_{I_{1}}\left(\sum x_{k}\right) \bar{S}_{I_{2}}\left(\sum x_{k}\right)+\bar{S}_{I_{1}}^{o} \bar{S}_{I_{2}}\left(\sum x_{k}\right)\right) .
\end{aligned}
$$

and thus the theorem.

As is proved by [29], suitable limits of the functions in Theorem 6 give the following theorem.

Theorem 11 (Rat- $B_{n}$, [29, Proposition 6.3]). Put

$$
\begin{aligned}
& u_{e_{i}-e_{j}}(x)=\frac{A}{\left(x_{i}-x_{j}\right)^{2}}, \quad u_{e_{i}+e_{j}}(x)=\frac{A}{\left(x_{i}+x_{j}\right)^{2}} \\
& v_{k}^{0}(x)=x_{k}^{-2}, \quad v_{k}^{1}(x)=x_{k}^{2}, \quad v_{k}^{2}(x)=x_{k}^{4}, \quad v_{k}^{3}(x)=x_{k}^{6} .
\end{aligned}
$$

Then (5.7) holds with

$$
\begin{aligned}
& T_{I}=(-1)^{\# I-1}\left(C S_{I}^{o}-C_{0} T_{I}^{o}\left(v^{0}\right)-C_{1} S_{I}\left(v^{1}\right)-C_{2} S_{I}\left(v^{2}\right)\right. \\
&+2 C_{2} \sum_{I_{1} \amalg I_{2}=I}\left(S_{I_{1}}\left(v^{1}\right) S_{I_{2}}^{o}+S_{I_{1}}^{o} S_{I_{2}}\left(v^{1}\right)\right)-2 C_{3} S_{I}^{o}\left(v^{3}\right) \\
&+C_{3} \sum_{I_{1} \amalg I_{2}=I}\left(S_{I_{1}}\left(v^{1}\right) S_{I_{2}}\left(v^{1}\right)+2 S_{I_{1}}\left(v^{2}\right) S_{I_{2}}^{o}+2 S_{I_{1}}^{o} S_{I_{2}}\left(v^{2}\right)\right) \\
&\left.-24 C_{3} \sum_{I_{1} \amalg I_{2} \amalg I_{3}=I}\left(S_{I_{1}}\left(v^{1}\right) S_{I_{2}}^{o} S_{I_{3}}^{o}+S_{I_{1}}^{o} S_{I_{2}}\left(v^{1}\right) S_{I_{3}}^{o}+S_{I_{1}}^{o} S_{I_{2}}^{o} S_{I_{3}}\left(v^{1}\right)\right)\right), \\
& T_{I}^{o}\left(v^{0}\right)=\sum_{\nu=1}^{\# I} \sum_{I_{1} \amalg \cdots \amalg I_{\nu}=I}(-A)^{\nu-1}(\nu-1) ! \cdot S_{I_{1}}\left(v^{0}\right) \cdots S_{I_{\nu}}\left(v^{0}\right) .
\end{aligned}
$$

Definition 5. We define some potential functions as specializations of potential functions in Definition 3.

(Trig- $A_{n-1}$-bry-reg) Trigonometric potential of type $A_{n-1}$ with regular boundary conditions is (Trig- $A_{n-1}$-bry) with $C_{2}=C_{3}=0$.

(Trig- $A_{n-1}$ ) Trigonometric potential of type $A_{n-1}$ is (Trig- $A_{n-1}$-bry) with $C_{0}=C_{1}=C_{2}=$ $C_{3}=0$.

(Trig- $B C_{n}$-reg) Trigonometric potential of type $B C_{n}$ with regular boundary conditions is (Trig$\left.B_{n}\right)$ with $C_{2}=C_{3}=0$.

(Toda- $D_{n}$-bry) Toda potential of type $D_{n}$ with boundary conditions is (Toda- $B_{n}^{(1)}$-bry) with $C_{0}=C_{1}=0$.

(Toda- $B_{n}^{(1)}$ ) Toda potential of type $B_{n}^{(1)}$ is (Toda- $B_{n}^{(1)}$-bry) with $C_{2}=C_{3}=0$.

(Toda- $D_{n}^{(1)}$ ) Toda potential of type $D_{n}^{(1)}$ is (Toda- $D_{n}^{(1)}$-bry) with $C_{0}=C_{1}=C_{2}=C_{3}=0$.

(Toda- $A_{n-1}$ ) Toda potential of type $A_{n-1}$ is (Toda- $C_{n}^{(1)}$ ) with $C_{0}=C_{1}=C_{2}=C_{3}=0$.

(Toda- $B C_{n}$ ) Toda potential of type $B_{n}$ is (Toda- $C_{n}^{(1)}$ ) with $C_{0}=C_{1}=0$.

(Ellip- $D_{n}$ ) Elliptic potential of type $D_{n}$ is (Ellip- $B_{n}$ ) with $C_{0}=C_{1}=C_{2}=C_{3}=0$.

(Trig- $D_{n}$ ) Trigonometric potential of type $D_{n}$ is (Trig- $B_{n}$ ) with $C_{0}=C_{1}=C_{2}=C_{3}=0$. 
(Rat- $\left.D_{n}\right)$ Rational potential of type $D_{n}$ is $\left(\right.$ Rat- $\left.B_{n}\right)$ with $C_{0}=C_{1}=C_{2}=C_{3}=0$.

(Toda- $D_{n}$ ) Toda potential of type $D_{n}$ is (Toda- $B_{n}^{(1)}$-bry) with $C_{0}=C_{1}=C_{2}=C_{3}=0$.

(Rat- $\left.B_{n}-2\right)$ Rational potential of type $B_{n}-2$ is (Rat- $\left.B_{n}\right)$ with $C_{2}=C_{3}=0$.

(Rat- $A_{n-1}$-bry2) Rational potential of type $A_{n-1}$ with 2-boundary conditions is (Rat- $A_{n-1}$-bry) with $C_{2}=C_{3}=0$. In this case, we may assume $C_{0}=0$ or $C_{1}=0$ by the transformation $x_{k} \mapsto x_{k}+c(k=1, \ldots, n)$ with a suitable $c \in \mathbb{C}$.

Then we have the following diagrams for $n \geq 3$. Note that we don't write all the arrows in the diagrams (ex. (Toda- $D_{n}$-bry) $\rightarrow\left(\right.$ Toda- $\left.B C_{n}\right)$ ) and the meaning of the symbol $\stackrel{5: 3}{\Rightarrow}$ is same as in the diagram for type $B_{2}$. Namely, 5 parameters (coupling constant) in the potential are reduced to 3 parameters by a certain restriction.

\section{Hierarchy of Elliptic-Trigonometric-Rational Integrable Potentials}

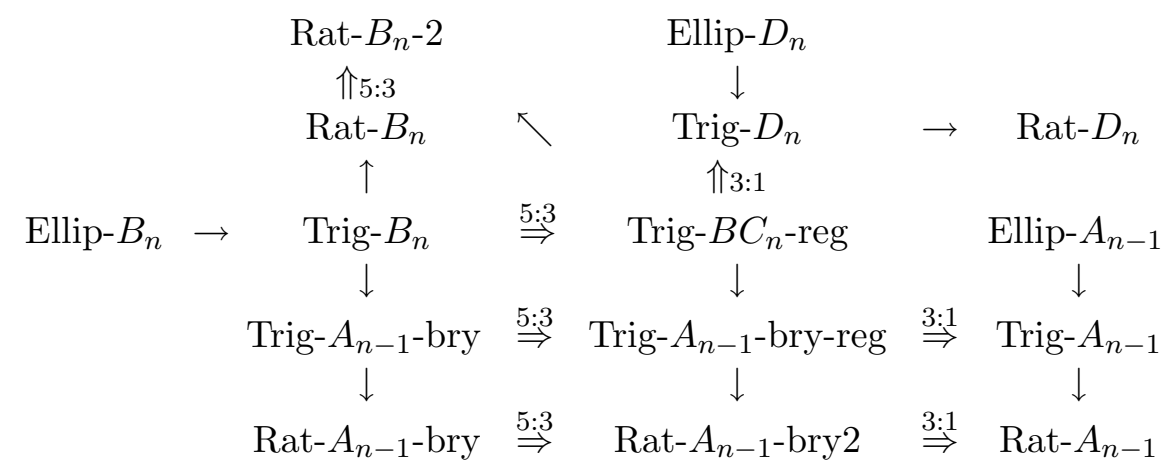

Hierarchy of Toda Integrable Potentials

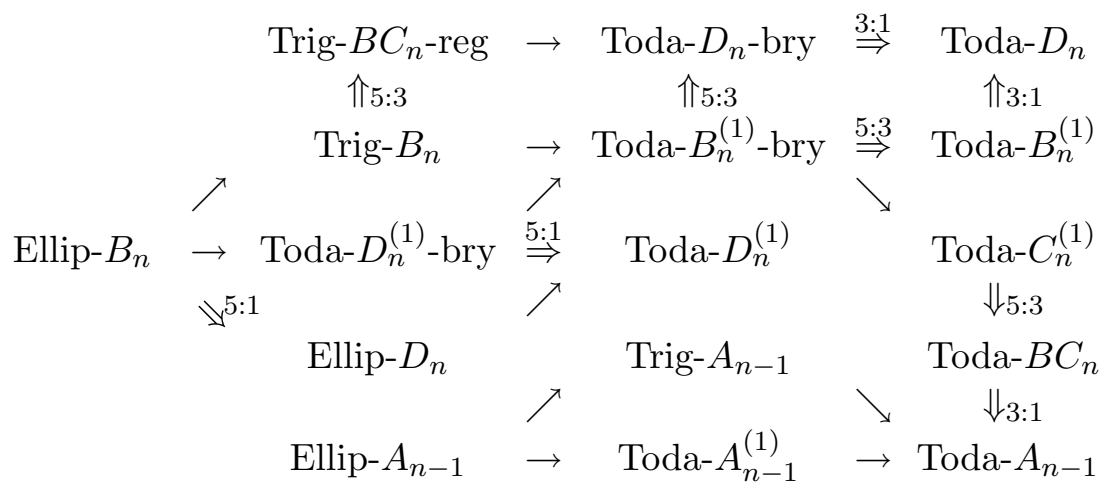

\section{$6 \quad$ Type $D_{n}(n \geq 3)$}

Theorem 12 (Type $D_{n}$ ). The Schrödinger operators (Ellip- $\left.D_{n}\right)$, (Trig- $\left.D_{n}\right)$, (Rat- $\left.D_{n}\right)$, (Toda$\left.D_{n}^{(1)}\right)$ and (Toda- $\left.D_{n}\right)$ are in the commutative algebra of differential operators generated by $P_{1}, P_{2}, \ldots, P_{n-1}$ and $\Delta_{\{1, \ldots, n\}}$ which are the corresponding operators for (Ellip- $B_{n}$ ), (Trig- $B_{n}$ ), (Rat- $\left.B_{n}\right)$, (Toda- $D_{n}^{(1)}$-bry), (Toda- $D_{n}$-bry) with $C_{0}=C_{1}=C_{2}=C_{3}=0$, respectively.

Proof. This theorem is proved by [29] in the cases $\left(\right.$ Ellip- $\left.D_{n}\right)$, (Trig- $\left.D_{n}\right)$, (Rat- $\left.D_{n}\right)$. Other two cases have been defined by suitable analytic continuation and therefore the claim is clear. 
Remark 10. In the above theorem we have $P_{n}=\Delta_{\{1, \ldots, n\}}^{2}$ because $q_{I}^{o}=0$ if $I \neq \varnothing$. Then $\left[P_{j}, P_{n}\right]=0$ implies $\left[P_{j}, \Delta_{\{1, \ldots, n\}}\right]=0$.

Hierarchy of Integrable Potentials of Type $D_{n}(n \geq 3)$

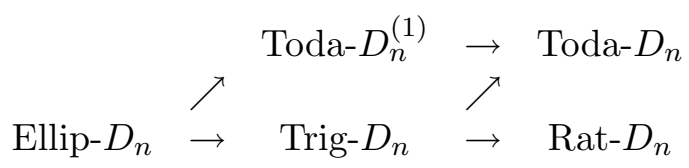

\section{Classical limits}

For functions $f(\xi, x)$ and $g(\xi, x)$ of $(\xi, x)=\left(\xi_{1}, \ldots, \xi_{n}, x_{1}, \ldots, x_{n}\right)$, we define their Poisson bracket by

$$
\{f, g\}=\sum_{k=1}^{n}\left(\frac{\partial f}{\partial \xi_{k}} \frac{\partial g}{\partial x_{k}}-\frac{\partial g}{\partial \xi_{k}} \frac{\partial f}{\partial x_{k}}\right) .
$$

\section{Theorem 13. Put}

$$
\bar{P}(\xi, x)=-\frac{1}{2} \sum_{k=1}^{n} \xi_{k}^{2}+R(x) .
$$

Then for the integrable potential function $R(x)$ given in this note, the functions $\bar{P}_{k}(\xi, x)$ and $\bar{\Delta}_{\{1, \ldots, n\}}(\xi, x)$ of $(\xi, x)$ defined by replacing $\partial_{\nu}$ by $\xi_{\nu}(\nu=1, \ldots, n)$ in the definitions of $P_{k}$ and $\Delta_{\{1, \ldots, n\}}$ in Sections 3, 4 and 5 satisfy

$$
\left\{\bar{P}_{i}(\xi, x), \bar{P}_{j}(\xi, x)\right\}=\left\{\bar{P}(\xi, x), \bar{P}_{k}(\xi, x)\right\}=0 \quad \text { for } \quad 1 \leq i<j \leq n \quad \text { and } \quad 1 \leq k \leq n .
$$

Hence $\bar{P}(\xi, x)$ are Hamiltonians of completely integrable dynamical systems.

Moreover if the potential function $R(x)$ is of type $D_{n}$, then

$$
\left\{\bar{\Delta}_{\{1, \ldots, n\}}(\xi, x), \bar{P}_{k}(\xi, x)\right\}=\left\{\bar{\Delta}_{\{1, \ldots, n\}}(\xi, x), \bar{P}(\xi, x)\right\}=0 \quad \text { for } \quad 1 \leq k \leq n .
$$

Proof. If $R(x)$ is a potential function of (Ellip- $A_{n-1}$ ), (Ellip- $B_{n}$ ) or (Ellip- $D_{n}$ ), the claim is proved in [29, 32]. Since the claim keeps valid under suitable holomorphic continuations with respect to the parameters which are given in the former sections, we have the theorem.

Remark 11. Since our operators $P_{k}$ are expressed by operators $P_{k}^{\nu}=\sum_{i} p_{k, i}^{\nu}(x) q_{k, i}^{\nu}(\partial)$ such that the polynomials $q_{k, i}^{\nu}(\partial)$ satisfy $\left[p_{k, i}^{\nu}(x), q_{k, i}^{\nu}(\partial)\right]=0$, there is no ambiguity in the definition of the classical limits by replacing $\partial_{\nu}$ by $\xi_{\nu}$. In another word, if we have given the above integrals $\bar{P}_{j}(x, \xi)$ of the classical limit, we have a natural unique quantization of them.

\section{Analogue for one variable}

Putting $n=1$ for the Schrödinger operator $P$ of type $A_{n}$ in Section 3 or of type $B_{n}$ in Section 5 , we examine the ordinary differential equation $P u=C u$ with $C \in \mathbb{C}$ (cf. [41, $\S 10.6]$ ). We will write the operators $Q=P-C$.

(Ellip- $B_{1}$ ) The Heun equation (cf. [32, § 8], [41, pp. 576])

$$
-\frac{1}{2} \frac{d^{2}}{d t^{2}}+\sum_{j=0}^{3} C_{j \wp}\left(t+\omega_{j}\right)-C .
$$


(Ellip- $\left.A_{1}\right) \quad$ The Lamé equation

$$
-\frac{1}{2} \frac{d^{2}}{d t^{2}}+A \wp(t)-C
$$

(Trig- $B C_{1}$-reg) The Gauss hypergeometric equation

$$
-\frac{1}{2} \frac{d^{2}}{d t^{2}}+\frac{C_{0}}{\sinh ^{2} \lambda t}+\frac{C_{1}}{\sinh ^{2} 2 \lambda t}-C .
$$

(Trig- $\left.A_{1}\right) \quad$ The Legendre equation

$$
-\frac{1}{2} \frac{d^{2}}{d t^{2}}+\frac{C_{0}}{\sinh ^{2} \lambda t}-C
$$

(Trig- $\left.B_{1}\right)$ with $C_{0}=C_{1}=C_{3}=0$. The (Modified) Mathieu equation

$$
-\frac{1}{2} \frac{d^{2}}{d t^{2}}+C_{2} \cosh 2 \lambda t-C
$$

(Rat- $\left.B_{1}-2\right) \quad$ Equation of the paraboloid of revolution

$$
-\frac{1}{2} \frac{d^{2}}{d t^{2}}+\frac{C_{0}}{t^{2}}+C_{1} t^{2}-C
$$

This is the Weber equation if $C_{0}=0$. With $s=t^{2}$ and using the unknown function $t^{\frac{1}{2}} u$, the above equation is reduced to the Whittaker equation:

$$
-\frac{1}{2} \frac{d^{2}}{d s^{2}}+\frac{C_{0}^{\prime}}{s^{2}}+\frac{C_{1}^{\prime}}{s}-C^{\prime}
$$

(Rat- $A_{0}$-bry2) with $C_{2}=C_{3}=0$ :

$$
-\frac{1}{2} \frac{d^{2}}{d t^{2}}+C_{0} t+C_{1} t^{2}-C
$$

If $C_{1} \neq 0$, this is transformed into the Weber equation under the coordinate $s=t+C_{2} /\left(2 C_{1}\right)$. If $C_{1}=0$, this is the Stokes equation which is reduced to the Bessel equation. In particular, the Airy equation corresponds to $C=C_{1}=0$.

(Toda- $B C_{1}$ )

$$
-\frac{1}{2} \frac{d^{2}}{d t^{2}}+C_{0} e^{-2 t}+C_{1} e^{-4 t}-C
$$

which is transformed into (Rat- $\left.B_{1}-2\right)$ by putting $s=e^{-t}$. In particular (Toda- $\left.A_{1}\right)$

$$
-\frac{1}{2} \frac{d^{2}}{d t^{2}}+C_{0} e^{-2 t}-C
$$

is reduced to the Bessel equation.

$\left(\right.$ Rat- $\left.A_{1}\right)$ the Bessel equation

$$
-\frac{1}{2} \frac{d^{2}}{d t^{2}}+\frac{C_{0}}{t^{2}}-C
$$


In fact, the equation $-u^{\prime \prime} / 2+C_{0} u / t^{2}=C u$ is equivalent to

$$
\left(\frac{d^{2}}{d t^{2}}+\frac{1}{t} \frac{d}{d t}-\frac{2 C_{0}+1 / 4}{t^{2}}+2 C\right) t^{-1 / 2} u=0
$$

since

$$
t^{-\frac{1}{2}} \circ \frac{d^{2}}{d t^{2}} \circ t^{\frac{1}{2}}=\frac{d^{2}}{d t^{2}}+\frac{1}{t} \frac{d}{d t}-\frac{1}{4 t^{2}}
$$

Hence if $C \neq 0$, the function $v=t^{-1 / 2} u$ satisfies the following Bessel equation with $s=\sqrt{-2 C} t$ :

$$
\frac{d^{2} v}{d s^{2}}+\frac{1}{s} \frac{d v}{d s}-\left(1-\frac{C_{0}+1 / 8}{C s^{2}}\right) v=0
$$

\section{Hierarchy of ordinary differential equations}

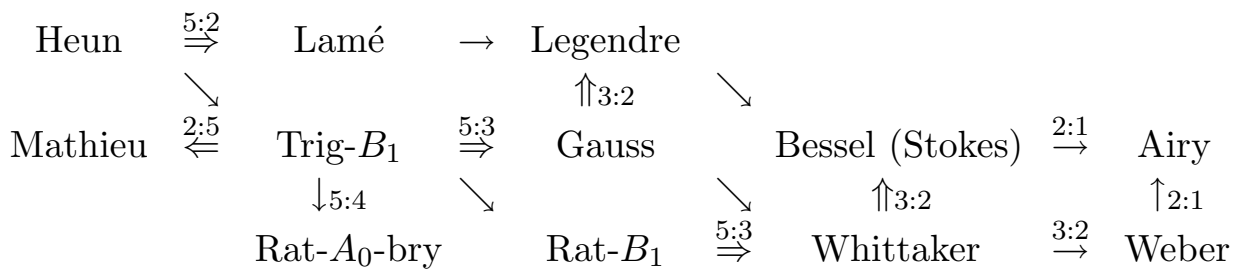

\section{A classification}

We present a conjecture which characterizes the systems listed in this note.

Let $P$ be the Schrödinger operator with the expression (1.1) and consider the condition

$$
\text { there exist } P_{1}, \ldots, P_{n} \text { such that }\left\{\begin{array}{l}
P \in \mathbb{C}\left[P_{1}, \ldots, P_{n}\right], \\
{\left[P_{i}, P_{j}\right]=0 \quad(1 \leq i<j \leq n),} \\
\sigma\left(P_{k}\right)=\sum_{1 \leq j_{1}<\cdots<j_{k} \leq n} \xi_{j_{1}}^{2} \cdots \xi_{j_{k}}^{2} \quad(1 \leq k \leq n) .
\end{array}\right.
$$

Note that all the completely integrable systems given in Sections 3, 4 or 5 satisfy this condition.

Conjecture. Suppose P satisfies (9.1). Under a suitable affine transformation of the coordinate $x \in \mathbb{C}^{n}$ which keeps the algebra $\mathbb{C}\left[\sum_{k=1}^{n} \partial_{k}^{2}, \sum_{k=1}^{n} \partial_{k}^{4}, \ldots, \sum_{k=1}^{n} \partial_{k}^{2 n}\right]$ invariant, $P$ is transformed into an integrable Schrödinger operator studied in Sections 3, 4 or 5, (namely $u_{i j}^{ \pm}$, $v_{k}$ and $w_{k}$ in (1.3) are suitable analytic continuations of the corresponding functions of the invariant elliptic systems) or in general a direct sum of such operators and/or trivial operators

$$
\frac{d^{2}}{d x^{2}}+v(x)
$$

with arbitrary functions $v(x)$ of one variable.

Here the direct sum of the two operators $P_{j}\left(x, \partial_{x}\right)=\sum_{\alpha \in\{0,1, \ldots\}^{n_{j}}} a_{\alpha}(x) \partial_{x}^{\alpha}$ of $x \in \mathbb{C}^{n_{j}}$ for $j=1,2$ means the operator $P_{1}\left(x, \partial_{x}\right)+P_{2}\left(y, \partial_{y}\right)$ of $(x, y) \in \mathbb{C}^{n_{1}+n_{2}}$.

We review known conditions assuring this conjecture and give another condition (cf. Theorem 19 and Remark 17). We also review related results on the classification of completely integrable quantum systems associated with classical root systems. 
Remark 12. The condition

there exists $P_{2}$ such that $\left[P, P_{2}\right]=0$ and $\sigma\left(P_{2}\right)=\sum_{1 \leq i<j \leq n} \xi_{i}^{2} \xi_{j}^{2}$

may be sufficient to assure the claim of the conjecture.

Remark 13 (Type $\boldsymbol{A}_{\mathbf{2}}$ ). If $n=2$ and if there exists $P_{3}$ satisfying

$$
\sigma\left(P_{3}\right)=\xi_{1} \xi_{2}+\xi_{2} \xi_{3}+\xi_{3} \xi_{1} \quad \text { and } \quad\left[P, P_{3}\right]=\left[\partial_{1}+\partial_{2}+\partial_{3}, P\right]=\left[\partial_{1}+\partial_{2}+\partial_{3}, P_{3}\right]=0,
$$

then Conjecture is true.

In fact this case is reduced to solving the equation

$$
\left|\begin{array}{lll}
u(x) & u^{\prime}(x) & 1 \\
v(y) & v^{\prime}(y) & 1 \\
w(z) & w^{\prime}(z) & 1
\end{array}\right|=0 \quad \text { for } \quad x+y+z=0
$$

for three unknown functions $u(t), v(t)$ and $w(t)$, which is solved by $[3,4]$. Here $u(t)=u_{e_{1}-e_{2}}(t)$, $v(t)=u_{e_{2}-e_{3}}(t)$ and $w(t)=u_{e_{1}-e_{3}}(-t)$.

\subsection{Pairwise interactions and meromorphy}

Theorem 14 ([40]). The potential function $R(x)$ of $P$ satisfying (9.1) is of the form

$$
R(x)=\sum_{\alpha \in \Sigma\left(B_{n}\right)^{+}} u_{\alpha}(\langle\alpha, x\rangle)
$$

with meromorphic functions $u_{\alpha}(t)$ of one variable.

Remark 14. i) The condition (9.2) assures

$$
R(x)=\sum_{\alpha \in \Sigma\left(B_{n}\right)^{+}} u_{\alpha}(\langle\alpha, x\rangle)+\sum_{1 \leq i<j<k \leq n} C_{i j k} x_{i} x_{j} x_{k}
$$

with $C_{i j k} \in \mathbb{C}$ and thus the above theorem is proved in the invariant case (cf. Section 9.2) by [32] or in the case of Type $B_{2}$ by [23] or in the case of Type $A_{n-1}$. This theorem is proved in [40] by using $\left[P, P_{2}\right]=\left[P, P_{3}\right]=0$.

ii) Suppose $n=2$ and the operators

$$
\begin{aligned}
& P=-\frac{1}{2}\left(\frac{\partial^{2}}{\partial x_{1}^{2}}+\frac{\partial^{2}}{\partial x_{2}^{2}}\right)+R\left(x_{1}, x_{2}\right), \\
& T=\sum_{i=0}^{m} c_{j} \frac{\partial^{m}}{\partial x_{1}^{i} \partial x_{2}^{m-i}}+\sum_{i+j \leq m-2} T_{i, j}\left(x_{1}, x_{2}\right) \frac{\partial^{i+j}}{\partial x_{1}^{i} \partial x_{2}^{j}}
\end{aligned}
$$

satisfy $[P, T]=0$ and $\sigma_{m}(T) \notin \mathbb{C}[\sigma(P)]$. Here $c_{j} \in \mathbb{C}$. Then $[31$, Theorem 8.1] shows that there exist functions $u_{\nu, i}(t)$ of one variable such that

$$
R\left(x_{1}, x_{2}\right)=\sum_{\nu=1}^{L} \sum_{i=0}^{m_{\nu}-1}\left(b_{\nu} x_{1}+a_{\nu} x_{2}\right)^{i} u_{\nu, i}\left(a_{\nu} x_{1}-b_{\nu} x_{2}\right)
$$

by putting

$$
\left(\xi \frac{\partial}{\partial \tau}-\tau \frac{\partial}{\partial \xi}\right) \sum_{i=0}^{m} c_{i} \xi^{m-i} \tau^{i}=\prod_{\nu=1}^{L}\left(a_{\nu} \xi-b_{\nu} \tau\right)^{m_{\nu}} .
$$

Here $\left(a_{\nu}, b_{\nu}\right) \in \mathbb{C}^{2} \backslash\{(0,0)\}$ and $a_{\nu} b_{\mu} \neq a_{\mu} b_{\nu}$ if $\mu \neq \nu$. 
Definition 6. By the expression (9.4), put

$$
S=\left\{\alpha \in \Sigma\left(B_{n}\right)^{+} ; u_{\alpha}^{\prime} \neq 0\right\}
$$

and let $W(S)$ be the Weyl group generated by $\left\{w_{\alpha} ; \alpha \in S\right\}$ and moreover put $\bar{S}=W(S) S$.

Theorem 15 ([23] for Type $\boldsymbol{B}_{\mathbf{2}},[40]$ in general). If the root system $\bar{S}$ has no irreducible component of rank one, then (9.2) assures that any function $u_{\alpha}(t)$ extends to a meromorphic function on $\mathbb{C}$.

Remark 15 ([32, (6.4)-(6.5)], [40, $\S 3])$. The condition $(9.2)$ is equivalent to

$$
S^{i j}=S^{j i} \quad(1 \leq i<j \leq n)
$$

with

$$
\begin{aligned}
S^{i j}= & \left(\partial_{i}^{2} v_{i}\left(x_{i}\right)+\sum_{\nu \in I(i, j)} \partial_{i}^{2}\left(u_{i \nu}^{+}\left(x_{i}+x_{\nu}\right)+u_{i \nu}^{-}\left(x_{i}-x_{\nu}\right)\right)\right)\left(u_{i j}^{+}\left(x_{i}+x_{j}\right)-u_{i j}^{-}\left(x_{i}-x_{j}\right)\right) \\
& +3\left(\partial_{i} v_{i}\left(x_{i}\right)+\sum_{\nu \in I(i, j)} \partial_{i}\left(u_{i \nu}^{+}\left(x_{i}+x_{\nu}\right)+u_{i \nu}^{-}\left(x_{i}-x_{\nu}\right)\right)\right)\left(\partial_{i} u_{i j}^{+}\left(x_{i}+x_{j}\right)-\partial_{i} u_{i j}^{-}\left(x_{i}-x_{j}\right)\right) \\
& +2\left(v_{i}\left(x_{i}\right)+\sum_{\nu \in I(i, j)}\left(u_{i \nu}^{+}\left(x_{i}+x_{\nu}\right)+u_{i \nu}^{-}\left(x_{i}-x_{\nu}\right)\right)\right)\left(\partial_{i}^{2} u_{i j}^{+}\left(x_{i}+x_{j}\right)-\partial_{i}^{2} u_{i j}^{-}\left(x_{i}-x_{j}\right)\right) \\
& +\sum_{\nu \in I(i, j)}\left(\partial_{i}^{2} u_{i \nu}^{+}\left(x_{i}+x_{\nu}\right)-\partial_{i}^{2} u_{i \nu}^{-}\left(x_{i}-x_{\nu}\right)\right)\left(u_{j \nu}^{+}\left(x_{j}+x_{\nu}\right)-u_{j \nu}^{-}\left(x_{j}-x_{\nu}\right)\right) .
\end{aligned}
$$

Here $I(i, j)=\{1,2, \ldots, n\} \backslash\{i, j\}$.

Lemma 5. Suppose $P$ satisfies (9.2) and (9.4). Let $S_{0}$ be a subset of $\bar{S}$ such that

$$
S_{0} \subset \sum_{i=1}^{m} \mathbb{R} e_{i} \quad \text { and } \quad \bar{S} \backslash S_{0} \subset \sum_{i=m+1}^{n} \mathbb{R} e_{i}
$$

with a suitable $m$. Then the Schrödinger operator

$$
P^{\prime}=-\frac{1}{2} \sum_{i=1}^{m} \partial_{i}^{2}+\sum_{\alpha \in S_{0} \cap S} u(\langle\alpha, x\rangle)
$$

on $\mathbb{R}^{m}$ admits a differential operator $P_{2}^{\prime}$ on $\mathbb{R}^{m}$ satisfying $\left[P^{\prime}, P_{2}^{\prime}\right]=0$ and $\sigma\left(P_{2}^{\prime}\right)=\sum_{1 \leq i<j \leq n}^{m} \xi_{i}^{2} \xi_{j}^{2}$, that is, the condition (9.2) with replacing $P$ by $P^{\prime}$.

Proof. This lemma clearly follows from the equivalent condition (9.5) given in Remark 15.

\subsection{Invariant case}

Theorem 16 ([24, 25, 29, 32]). Assume that $P$ in (1.1) is invariant under the Weyl group $W=W\left(A_{n-1}\right), W\left(B_{n}\right)$ or $W\left(D_{n}\right)$ with $n \geq 3$, or $W=W\left(B_{2}\right)$. If we have (1.2) with

$$
P_{1}=\partial_{1}+\partial_{2}+\cdots+\partial_{n} \quad \text { if } \quad W=W\left(A_{n-1}\right),
$$




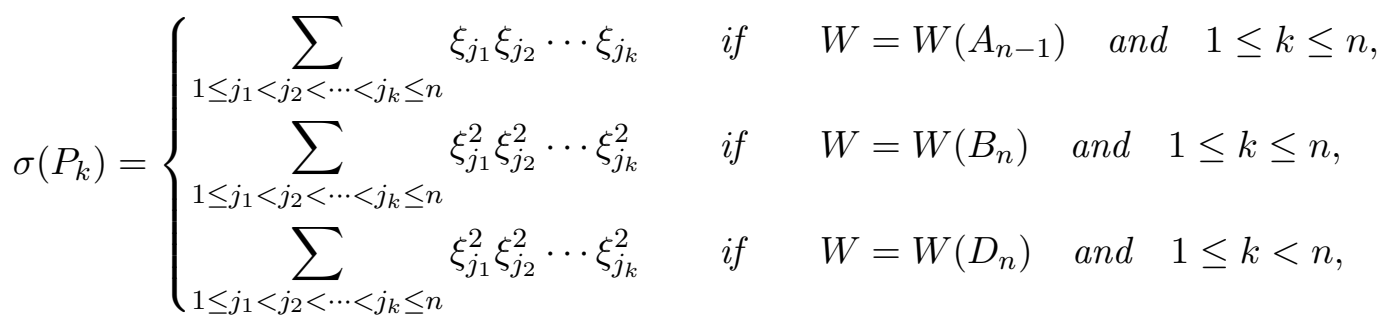

$$
\begin{aligned}
& \sigma\left(P_{n}\right)=\xi_{1} \xi_{2} \cdots \xi_{n} \quad \text { if } \quad W=W\left(D_{n}\right),
\end{aligned}
$$

Conjecture is true.

Remark 16. The condition

$$
\begin{aligned}
& {\left[P, P_{1}\right]=\left[P, P_{3}\right]=0 \quad \text { if } \quad W=W\left(A_{n-1}\right),} \\
& {\left[P, P_{2}\right]=0 \quad \text { if } \quad W=W\left(B_{n}\right) \quad \text { or } \quad W=W\left(D_{n}\right)}
\end{aligned}
$$

together with (9.4) is sufficient for the proof of this theorem.

\subsection{Enough singularities}

Put $\Xi=\left\{\alpha \in \Sigma\left(B_{n}\right)^{+} ; u_{\alpha}(t)\right.$ is not entire $\}$.

Theorem 17. i) ([23]) Suppose $n=2$ and let $\bar{S}$ be of type $B_{2}$. If $\# \Xi \geq 2$, then Conjecture is true.

ii) ([40]) If $\bar{S}$ is of type $A_{n-1}$ or of type $B_{n}$ and moreover the reflections $w_{\alpha}$ for $\alpha \in \Xi$ generate $W\left(A_{n-1}\right)$ or $W\left(B_{n}\right)$, respectively, then Conjecture is true.

This theorem follows from the following key Lemma.

Lemma 6 ([23, 37, 40]). Suppose (9.1) and moreover that there exist $\alpha$ and $\beta$ in $S$ such that $\alpha \neq \beta,\langle\alpha, \beta\rangle \neq 0$ and $u_{\alpha}(t)$ has a singularity at $t=t_{0}$. Then $u_{\alpha}\left(t-t_{0}\right)$ is an even function with a pole of order two at the origin and

$$
\begin{aligned}
& u_{w_{\alpha}(\beta)}\left(t-2 t_{0} \frac{\langle\alpha, \gamma\rangle}{\langle\alpha, \alpha\rangle}\right)=u_{\beta}(t) \quad \text { if } \quad w_{\alpha}(\beta) \in \Sigma\left(B_{n}\right)^{+}, \\
& u_{-w_{\alpha}(\beta)}\left(-t+2 t_{0} \frac{\langle\alpha, \gamma\rangle}{\langle\alpha, \alpha\rangle}\right)=u_{\beta}(t) \quad \text { if } \quad-w_{\alpha}(\beta) \in \Sigma\left(B_{n}\right)^{+} .
\end{aligned}
$$

Corollary 1. Suppose the assumption in Lemma 6.

i) If $u_{\alpha}(t)$ has another singularity at $t_{1} \neq t_{0}$, then

$$
u_{\gamma}\left(t+2\left(t_{1}-t_{0}\right) \frac{\langle\alpha, \gamma\rangle}{\langle\alpha, \alpha\rangle}\right)=u_{\gamma}(t) \quad \text { for } \quad \gamma \in S
$$

ii) Assume that $u_{\alpha}$ has poles at $0, t_{0}$ and $t_{1}$ such that $t_{0}$ and $t_{1}$ are linearly independent over $\mathbb{R}$. Then $u_{\beta}(t)$ is a doubly periodic function and therefore $u_{\beta}(t)$ has poles and hence $u_{\alpha}(t)$ is also a doubly periodic function. We may moreover assume that $u_{\beta}$ has a pole at 0 by a parallel transformation of the variable $x$.

Case I: Suppose $\alpha=e_{i}-e_{j}, \beta=e_{j}-e_{k}$ with $1 \leq i<j<k \leq n$.

$$
u_{e_{i}-e_{j}}(t)=u_{e_{j}-e_{k}}(t)=u_{e_{i}-e_{k}}(t)=C \wp\left(t ; 2 \omega_{1}, 2 \omega_{2}\right)+C^{\prime}
$$

with suitable $C, C^{\prime} \in \mathbb{C}$, which corresponds to (Ellip- $A_{2}$ ).

Case II: Suppose $\alpha=e_{i}-e_{j}$ and $\beta=e_{j}$ with $1 \leq i<j \leq n$.

Then $\left(u_{e_{i}-e_{j}}(t), u_{e_{i}+e_{j}}(x), u_{e_{i}}(t), u_{e_{j}}(t)\right)$ is (Ellip- $\left.B_{2}\right)$, (Ellip- $\left.B_{2}-\mathrm{S}\right)$ or (Ellip $\left.{ }^{d}-B_{2}\right)$. 
iii) If $\bar{S}$ is of type $A_{n-1}$ or $B_{n}$ or $D_{n}$ and one of $u_{\alpha}(t)$ is a doubly periodic function with poles, then $P$ transforms into (Ellip- $\left.A_{n-1}\right)$ or $\left(\right.$ Ellip- $B_{n}$ ) or (Ellip- $D_{n}$ ) under a suitable parallel transformation on $\mathbb{C}^{n}$.

Proof of Corollary 1. i) is a direct consequence of Lemma 6. iii) follows from ii). We have only to show ii).

Case I: It follows from (9.6) that $u_{\alpha}(t)=u_{\beta}(t)=u_{e_{i}-e_{k}}(t)$ and they are even functions. Let

$$
\Gamma_{2 \omega_{1}, 2 \omega_{2}}=\left\{2 m_{1} \omega_{1}+2 m_{2} \omega_{2} ; m_{1}, m_{2} \in \mathbb{Z}\right\}
$$

be the set of poles of $u_{\alpha}$. Then (9.7) implies $u_{\beta}\left(t+2 \omega_{1}\right)=u_{\beta}\left(t+2 \omega_{2}\right)=u_{\beta}(t)$. Since $2 \omega_{1}$ and $2 \omega_{2}$ are periods of $\wp(t)$ and there exists only one double pole in the fundamental domain defined by these periods, we have the claim.

Case II: It follows from (9.6) that $u_{e_{i}-e_{j}}(t)=u_{e_{i}+e_{j}}(t)$ and $u_{e_{i}}(t)=u_{e_{k}}(t)$ and they are even functions. Let $\Gamma_{2 \omega_{1}, 2 \omega_{2}}$ be the poles of $u_{e_{i}-e_{j}}(t)$. Then (9.7) means $u_{e_{i}}\left(t+2 \omega_{1}\right)=u_{e_{i}}\left(t+2 \omega_{2}\right)=$ $u_{e_{i}}(t)$. Considering the poles of $u_{e_{i}-e_{k}}(t)$ with (9.7), we have four possibilities of poles of $u_{e_{i}}$ :

(Case II-0): $\Gamma_{2 \omega_{1}, 2 \omega_{2}}$,

(Case II-1): $\Gamma_{2 \omega_{1}, 2 \omega_{2}} \bigcup\left(\omega_{1}+\Gamma_{2 \omega_{1}, 2 \omega_{2}}\right)$,

(Case II-2): $\Gamma_{2 \omega_{1}, 2 \omega_{2}} \bigcup\left(\omega_{2}+\Gamma_{2 \omega_{1}, 2 \omega_{2}}\right)$,

(Case II-3): $\Gamma_{2 \omega_{1}, 2 \omega_{2}} \bigcup\left(\omega_{1}+\Gamma_{2 \omega_{1}, 2 \omega_{2}}\right) \bigcup\left(\omega_{2}+\Gamma_{2 \omega_{1}, 2 \omega_{2}}\right)$.

Here we note that (Case II-1) changes into (Case II-2) if we exchange $\omega_{1}$ and $\omega_{2}$. Then we have

(Case II-0): $u_{e_{i}-e_{j}}\left(t+4 \omega_{1}\right)=u_{e_{i}-e_{j}}\left(t+4 \omega_{2}\right)=u(t)$,

(Case II-2): $u_{e_{i}-e_{j}}\left(t+4 \omega_{1}\right)=u_{e_{i}-e_{j}}\left(t+2 \omega_{2}\right)=u(t)$,

(Case II-3): $u_{e_{i}-e_{j}}\left(t+2 \omega_{1}\right)=u_{e_{i}-e_{j}}\left(t+2 \omega_{2}\right)=u(t)$.

Thus (Case II-0), (Case II-2) and (Case II-3) are reduced to (Ellip $\left.{ }^{d}-B_{2}\right)$, (Ellip- $B_{2}$-S) and (Ellip- $B_{2}$ ), respectively.

Let $\mathcal{H}$ be a finite set of mutually non-parallel vectors in $\mathbb{R}^{n}$ and suppose

$$
P=-\frac{1}{2} \sum_{j=1}^{n} \partial_{j}^{2}+R(x), \quad R(x)=\sum_{\alpha \in \mathcal{H}} C_{\alpha} \frac{\langle\alpha, \alpha\rangle}{\langle\alpha, x\rangle^{2}}+\tilde{R}(x) .
$$

Here $C_{\alpha}$ are nonzero complex numbers and $\tilde{R}(x)$ is real analytic at the origin. We assume that $\mathcal{H}$ is irreducible, namely,

$$
\begin{aligned}
& \mathbb{R}^{n}=\sum_{\alpha \in \mathcal{H}} \mathbb{R} \alpha, \\
& \varnothing \neq \forall \mathcal{H}^{\prime} \varsubsetneqq \mathcal{H} \Rightarrow \exists \alpha \in \mathcal{H}^{\prime} \quad \text { and } \quad \exists \beta \in \mathcal{H} \backslash \mathcal{H}^{\prime} \quad \text { with } \quad\langle\alpha, \beta\rangle \neq 0 .
\end{aligned}
$$

Definition 7. The potential function $R(x)$ of a Schrödinger operator is reducible if $R(x)$ and $\mathbb{R}^{n}$ is decomposed as $R(x)=R_{1}(x)+R_{2}(x)$ and $\mathbb{R}^{n}=V_{1} \oplus V_{2}$ such that

$$
0 \subsetneq V_{1} \subsetneq \mathbb{R}^{n}, \quad V_{2}=V_{1}^{\perp}, \quad \partial_{v_{2}} R_{1}(x)=\partial_{v_{1}} R_{2}(x)=0 \quad \text { for } \quad \forall v_{2} \in V_{2} \quad \text { and } \quad \forall v_{1} \in V_{1} .
$$

If $R(x)$ is not reducible, $R(x)$ is called to be irreducible.

Theorem 18 ([37]). Suppose $n \geq 2$ and there exists a differential operator $Q$ with $[P, Q]=0$ whose principal symbol does not depend on $x$ and is not a polynomial of $\sum_{i=0}^{n} \xi_{i}^{2}$. Put $W=$ $\left\{w_{\alpha} ; \alpha \in \mathcal{H}\right\}$. If

$2 C_{\alpha} \neq k(k+1) \quad$ for $\quad k \in \mathbb{Z} \quad$ and $\quad \alpha \in \mathcal{H}$,

then $W$ is a finite reflection group and $\sigma(Q)$ is $W$-invariant. 


\subsection{Periodic potentials}

The following theorem is a generalization of the result in [30].

Theorem 19. Assume $R(x)$ is of the form (9.4) with meromorphic functions $u_{\alpha}(t)$ on $\mathbb{C}$ and

$$
R\left(x+\frac{2 \pi \sqrt{-1} \alpha}{\langle\alpha, \alpha\rangle}\right)=R(x) \quad \text { for } \quad \alpha \in \Sigma\left(B_{n}\right)
$$

and moreover assume that

the root system $\bar{S}$ does not contain an irreducible component of type $B_{2}$ or even if $\bar{S}$ contains an irreducible component

$\bar{S}_{2}=\left\{ \pm e_{i} \pm e_{j}, \pm e_{i}, \pm e_{j}\right\}$ of type $B_{2}$, the origin $s=0$ is not an isolated essential singularity of $u_{\alpha}(\log s)$ for $\alpha \in \bar{S}_{2} \bigcap \Sigma\left(D_{n}\right)^{+}$.

Then Conjecture is true.

Remark 17. i) The integrable systems classified in this note which satisfy the assumption of Theorem 19 under a suitable coordinate system are (Ellip-*) and (Trig-*) and (Toda-*), which are the systems given in this note whose potential functions are not rational.

ii) The assumption (9.9) implies that $u_{\alpha}(\log s)$ is a meromorphic function on $\mathbb{C} \backslash\{0\}$ for any $\alpha \in \Sigma\left(B_{n}\right)^{+}$. It means that the corresponding Schrödinger operator is naturally defined on the Cartan subgroup of $S p(n, \mathbb{C})$ with a meromorphic potential function.

Lemma 7. Assume $n=2,(9.2),(9.9), \bar{S}$ is of type $B_{2}$ and moreover $u_{\alpha}(\log s)$ are holomorphic for $\alpha \in \Sigma\left(B_{2}\right)^{+}$and $0<|s| \ll 1$. If the origin is at most a pole of $u_{\beta}(\log s)$ for $\beta \in \Sigma\left(D_{2}\right)^{+}$, the origin is also at most a pole of $u_{\alpha}(\log s)$ for $\alpha \in \Sigma\left(B_{2}\right)^{+}$.

Proof. Use the notation as in (4.2). Put

$$
\begin{array}{lc}
u^{-}(\log s)=U_{0}^{-}+\sum_{\nu=r}^{\infty} \nu U_{\nu}^{-} s^{\nu}, & u^{+}(\log s)=U_{0}^{+}+\sum_{\nu=m}^{\infty} \nu U_{\nu}^{+} s^{\nu}, \\
v(\log s)=V_{0}+\sum_{\nu=-\infty}^{\infty} \nu V_{\nu} s^{\nu}, & w(\log s)=W_{0}+\sum_{\nu=-\infty}^{\infty} \nu W_{\nu} s^{\nu} .
\end{array}
$$

with $U_{\nu}^{-}, U_{\nu}^{+}, V_{\nu}, W_{\nu} \in \mathbb{C}, r m \neq 0$ and $\left(U_{r}^{-}, U_{m}^{+}\right) \neq 0$. Then as is shown in [30] the condition for the existence of $T(x, y)$ in (4.3) is equivalent to

$$
p q(2 p-q)(p-q)\left(V_{2 p-q} U_{q-p}^{+}+V_{q} U_{p-q}^{-}+W_{q-2 p} U_{p}^{+}-W_{q} U_{p}^{-}\right)=0 \quad \text { for } \quad p, q \in \mathbb{Z} .
$$

Hence if $p<r$ and $p<m$,

$$
p(p-k)(p+k) k\left(V_{p+k} U_{-k}^{+}+V_{p-k} U_{k}^{-}\right)=0 \quad \text { for } \quad k \in \mathbb{Z} .
$$

Case $U_{r}^{-} \neq 0$ : Put $k=r$. Suppose $q$ is negative with a sufficiently large absolute value. Then $V_{q}=\left(-U_{-r}^{+} / U_{r}^{-}\right) V_{q+2 r}$, which implies $V_{q}=0$ since $\sum_{\nu=-\infty}^{\infty} \nu V_{\nu} s^{\nu}$ converges for $0<|s| \ll 1$.

Suppose $q$ is negative with a sufficiently large absolute value compared to $p$. Then by the relation $W_{q-2 p} U_{p}^{+}-W_{q} U_{p}^{-}=0$ we similarly conclude $W_{q}=0$.

Case $U_{m}^{+} \neq 0$ : Putting $k=-m$, we have the same conclusion as above in the same way. 
Proof of Theorem 19. Lemma 5 assures that we may assume $\bar{S}$ is an irreducible root system. We may moreover assume that the rank of $\bar{S}$ is greater than one.

Suppose that there exists $\gamma \in S$ such that the origin is neither a removable singularity nor an isolated singularity of $u_{\gamma}(\log s)$. Then $u_{\gamma}(t)$ is a doubly periodic function with poles. Owing to Corollary 1, $\sum_{\alpha \in S_{0}} u_{\alpha}(\langle\alpha, x\rangle)$ is reduced to the potential function of (Ellip- $\left.A_{n-1}\right)$ or (Ellip- $\left.B_{n}\right)$ or (Ellip- $\left.D_{n}\right)$.

Thus we may assume that the origin is a removable singularity or an isolated singularity of $u_{\alpha}(\log s)$ for any $\alpha \in S$.

Let $\alpha, \beta \in S \bigcap \Sigma\left(D_{n}\right)$ with $\alpha \neq \beta$ and $\langle\alpha, \beta\rangle \neq 0$. Put $\gamma=w_{\alpha} \beta$ or $\gamma=-w_{\alpha} \beta$ so that $\gamma \in \Sigma\left(D_{n}\right)^{+}$. Then [30] shows that $u(t)=u_{\alpha}(t), v(t)=u_{\beta}(t)$ and $w(t)=u_{\gamma}(-t)$ satisfy (9.3). Then Remark 13 says that the origin is at most a pole of $u(\log s), v(\log s)$ and $w(\log s)$.

Let $\alpha \in S \cap \Sigma\left(D_{n}\right)$ and $\beta \in S \backslash \Sigma\left(D_{n}\right)$ with $\langle\alpha, \beta\rangle \neq 0$. Let $W$ be the reflection group generated by $w_{\alpha}$ and $w_{\beta}$ and put $S^{o}=W\{\alpha, \beta\} \cap \Sigma\left(B_{n}\right)$. Then [30] also shows that

$$
R(x)=\sum_{\gamma \in S^{o}} u_{\gamma}(\langle\gamma, x\rangle)
$$

defines an integrable potential function of type $B_{2}$. Hence Lemma 7 assures that the origin is at most a pole of $u_{\alpha}(\log s)$ for $\alpha \in S^{o}$.

Since $S$ is irreducible, the origin is at most a pole of $u_{\alpha}(\log s)$ for $\alpha \in S$. Then Theorem 19 follows from [30].

\subsection{Uniqueness}

We give some remarks on the operator which commutes with the Schrödinger operator $P$.

Remark 18 ([32, Lemma 3.1 ii)]). If differential operators $Q$ and $Q^{\prime}$ satisfy $\left[Q, Q^{\prime}\right]=0$, $\sigma\left(Q^{\prime}\right)=\sum_{j=1}^{n} \xi_{j}^{N}$ and $\operatorname{ord}(Q) \leq N-2$, then $Q$ has a constant principal symbol, that is, $\sigma(Q)$ does not depend on $x$.

Hence if there exist differential operators $Q_{1}, \ldots, Q_{n}$ with constant principal symbols such that $\sigma\left(Q_{1}\right), \ldots, \sigma\left(Q_{n}\right)$ are algebraically independent and moreover they satisfy $\left[Q_{i}, Q_{j}\right]=0$ for $1 \leq i<j \leq n$, then any operator $Q$ satisfying $\left[Q, Q_{j}\right]=0$ for $j=1, \ldots, n$ has a constant principal symbol. In particular, if a differential operator $Q$ satisfies $\left[Q, P_{k}\right]=0$ for $P_{k}$ in (1.2) and (1.4) with $k=1, \ldots, n$, then $\sigma(Q)$ does not depend on $x$.

Remark 19. Assume that a differential operator $Q$ commutes with a Schrödinger operator $P$ and moreover assume that there exist linearly independent vectors $c_{j} \in \mathbb{C}^{n}$ for $j=1, \ldots, n$ such that the operator is invariant under the parallel transformations $x \mapsto x+c_{j}$ for $j=1, \ldots, n$. Then $\sigma(Q)$ does not depend on $x$ (cf. [32, Lemma $3.1 \mathrm{i}$ )]).

Furthermore assume that $P$ is of type (Ellip- $F$ ) or (Trig- $F$ ) or (Rat- $F$ ) with $F=A_{n-1}$ or $B_{n}$ or $D_{n}$. If the condition (9.8) holds or $Q$ is $W(F)$-invariant, it follows from Theorem 18 or [29, Proposition 3.6] that $Q$ is in the ring $\mathbb{C}\left[P_{1}, \ldots, P_{n}\right]$ generated by the $W(F)$-invariant commuting differential operators. If the condition (8.11) is not valid, $\sigma(Q)$ is not necessarily $W(F)$-invariant (cf. $[7,35,39])$.

Remark 20 ([32, Theorem 3.2]). Let $P$ be the Schrödinger operator in Theorem 16 . Under the notation in Theorem 16 suppose $P_{k}$ are $W$-invariant for $1 \leq k \leq n$. Then the ring $\mathbb{C}\left[P_{1}, \ldots, P_{n}\right]$ is uniquely determined by $P$ and $Q$, where $Q=P_{3}$ if $W=W\left(A_{n-1}\right)$ and $Q=P_{2}$ if $W=W\left(B_{n}\right)$ or $W\left(D_{n}\right)$. 
Remark 21. If $P_{c}=-(1 / 2) \sum_{j=1}^{n} \partial_{j}^{2}+c R(x)$ is a Schrödinger operator with a coupling constant $c \in \mathbb{C}$ such that $P_{c}$ admits a non-trivial commuting differential operator $Q_{c}$ of order four for any $c \in \mathbb{C}$, then the operator $P_{c}$ may be a system stated in Conjecture under a suitable coordinate system.

The following example satisfies neither this condition nor the condition (9.8). It does not admit commuting differential operators (1.2) satisfying (1.4) if $m \neq 0,-1$.

Example 2. It is shown in $[6,35]$ that the Schrödinger operator

$$
P=-\frac{1}{2} \sum_{j=1}^{n} \frac{\partial^{2}}{\partial x_{j}^{2}}+\sum_{1 \leq i<j<n} \frac{m(m+1)}{\left(x_{i}-x_{j}\right)^{2}}+\sum_{i=1}^{n-1} \frac{m+1}{\left(x_{i}-\sqrt{m} x_{n}\right)^{2}}
$$

is completely integrable for any $m$ and algebraically integrable if $m$ is an integer.

The following example shows that the Schrödinger operator $P$ does not necessarily determine the commuting system $\mathbb{C}\left[P_{1}, \ldots, P_{n}\right]$.

Example 3. Let $\alpha, \beta, \gamma$ and $\lambda$ be complex numbers. Put $\left(A_{0}, A_{1}, C_{0}, C_{1}\right)=(\alpha, \gamma / 2-\lambda / 2, \beta, \lambda)$ for $\left(\right.$ Rat- $\left.B_{2}-\mathrm{S}\right)$ in Theorem 3 (cf. [32, Remark 3.7]). Then the Schrödinger operator

$$
P_{\alpha, \beta, \gamma}=-\frac{1}{2}\left(\frac{\partial^{2}}{\partial x^{2}}+\frac{\partial^{2}}{\partial y^{2}}\right)+\left(x^{2}+y^{2}\right)\left(\frac{2 \alpha}{\left(x^{2}-y^{2}\right)^{2}}+\frac{\beta}{x^{2} y^{2}}+\gamma\right)
$$

commutes with

$$
\begin{aligned}
Q_{\alpha, \beta, \gamma, \lambda}= & \left(\frac{\partial^{2}}{\partial x \partial y}+\frac{4 \alpha x y}{\left(x^{2}-y^{2}\right)^{2}}-2(\gamma-\lambda) x y\right)^{2}-2\left(\frac{\beta}{y^{2}}+\lambda y^{2}\right) \frac{\partial^{2}}{\partial x^{2}}-2\left(\frac{\beta}{x^{2}}+\lambda x^{2}\right) \frac{\partial^{2}}{\partial y^{2}} \\
& +4\left(\frac{\beta}{x^{2}}+\lambda x^{2}\right)\left(\frac{\beta}{y^{2}}+\lambda y^{2}\right)+\frac{16 \alpha \lambda x^{2} y^{2}+16 \alpha \beta}{\left(x^{2}-y^{2}\right)^{2}}+8 \lambda(\gamma-\lambda) x^{2} y^{2}
\end{aligned}
$$

for any $\lambda \in \mathbb{C}$. Note that $\left[Q_{\alpha, \beta, \gamma, \lambda}, Q_{\alpha, \beta, \gamma, \lambda^{\prime}}\right] \neq 0$ if $\lambda \neq \lambda^{\prime}$ and these operators are $W\left(B_{2}\right)$ invariant. The half of the coefficient of the term $\lambda$ of $Q_{\alpha, \beta, \gamma, \lambda}$ considered as a polynomial function of $\lambda$ is

$$
S_{\alpha, \beta, \gamma}=-\left(y \frac{\partial}{\partial x}-x \frac{\partial}{\partial y}\right)^{2}+2 \alpha\left(\frac{x y}{(x-y)^{2}}-\frac{x y}{(x+y)^{2}}\right)+2 \beta\left(\frac{y^{2}}{x^{2}}+\frac{x^{2}}{y^{2}}\right)+4 \gamma x^{2} y^{2} .
$$

In particular, $P=-(1 / 2)\left(\partial_{x}^{2}+\partial_{y}^{2}\right)+\gamma\left(x^{2}+y^{2}\right)$ commutes with $\partial_{x} \partial_{y}-2 \gamma x y$ and $x \partial_{y}-y \partial_{x}$.

Note that if $R(x)$ is a polynomial function on $\mathbb{C}^{n}$, the condition $\left[-(1 / 2) \sum_{j=1}^{n} \partial_{j}^{2}+R(x), Q\right]=0$ for a differential operator $Q$ implies that the coefficients of $Q$ are polynomial functions (cf. [32, Lemma 3.4]).

\subsection{Regular singularities}

Definition 8 ([16]). Put $\vartheta_{k}=t_{k} \partial / \partial t_{k}$ and $Y_{k}=\left\{t=\left(t_{1}, \ldots, t_{n}\right) \in \mathbb{C}^{n} ; t_{k}=0\right\}$. Then a differential operator $Q$ of the variable $t$ is said to have regular singularities along the set of walls $\left\{Y_{1}, \ldots, Y_{n}\right\}$ if

$$
Q=q\left(\vartheta_{1}, \ldots, \vartheta_{n}\right)+\sum_{k=1}^{n} t_{k} Q_{k}(t, \vartheta)
$$


Here $q$ is a polynomial of $n$ variables and $Q_{k}$ are differential operators with the form

$$
Q_{k}(t, \vartheta)=\sum a_{\alpha}(t) \vartheta_{1}^{\alpha_{1}} \cdots \vartheta_{n}^{\alpha_{n}}
$$

and $a_{\alpha}(t)$ are analytic at $t=0$. In this case we define

$$
\sigma_{*}(Q)=q\left(\xi_{1}, \ldots, \xi_{n}\right)
$$

and $\sigma_{*}(Q)$ is called the indicial polynomial of $Q$.

Theorem 20. Let $R(t)$ be a holomorphic function defined on a neighborhood of the origin of $\mathbb{C}^{n}$. Let $Q_{1}$ and $Q_{2}$ be differential operators of t which have regular singularities along the set of walls $\left\{Y_{1}, \ldots, Y_{n}\right\}$. Suppose $\sigma_{*}\left(Q_{1}\right)=\sigma_{*}\left(Q_{2}\right)$ and $\left[Q_{1}, P\right]=\left[Q_{2}, P\right]=0$ with the Schrödinger operator

$$
P=-\frac{1}{2}\left(\vartheta_{n}^{2}+\sum_{j=1}^{n-1}\left(\vartheta_{j+1}-\vartheta_{j}\right)^{2}\right)+R(t) .
$$

Then $Q_{1}=Q_{2}$.

Proof. Put $t_{j}=e^{-\left(x_{j}-x_{j+1}\right)}$ for $j=1, \ldots, n-1$ and $t_{n}=e^{-x_{n}}$. Then $\partial_{j}=\vartheta_{j+1}-\vartheta_{j}$ for $j=$ $1, \ldots, n-1$ and $\partial_{n}=-\vartheta_{n}$. Under the coordinate system $x=\left(x_{1}, \ldots, x_{n}\right)$ Remark 19 says that $Q_{1}-Q_{2}$ has a constant principal symbol, which implies $Q_{1}=Q_{2}$ because $\sigma_{*}\left(Q_{1}-Q_{2}\right)=0$.

A more general result than this theorem is given in [31]. The following corollary is a direct consequence of this theorem.

Corollary 2. Put $t_{j}=e^{-\lambda\left(x_{j}-x_{j+1}\right)}$ for $j=1, \ldots, n-1$ and $t_{n}=e^{-\lambda x_{n}}$. Suppose $P$ is the Schrödinger operator of type (Trig- $\left.A_{n-1}\right)$, (Trig- $A_{n-1}$-bry-reg), (Trig- $B C_{n}$-reg), (Trig- $D_{n}$ ), (Toda- $\left.A_{n-1}\right)$, (Toda- $\left.B C_{n}\right)$ or $\left(\right.$ Toda- $\left.D_{n}\right)$.

i) $P$ and $P_{k}$ for $k=1, \ldots, n$ have regular singularities along the set of walls $\left\{Y_{1}, \ldots, Y_{n}\right\}$.

ii) Let $Q$ be a differential operator which has regular singularities along the set of walls $\left\{Y_{1}, \ldots, Y_{n}\right\}$ and satisfies $[Q, P]=0$. If $\sigma_{*}(Q)=\sigma_{*}(\tilde{Q})$ for an operator $\tilde{Q} \in \mathbb{C}\left[P_{1}, \ldots, P_{n}\right]$, then $Q=\tilde{Q}$.

Remark 22. i) This corollary assures that certain radial parts of invariant differential operators on a symmetric space correspond to our completely integrable systems with regular singularities and the map $\sigma_{*}$ corresponds to the Harish-Chandra isomorphism (cf. [31]).

ii) The system (Trig- $B C_{n}$-reg) is Heckman-Opdam's hypergeometric system [11] of type $B C_{n}$. Since (Trig- $B C_{n}$-reg) is a generalization of Gauss hypergeometric system related to the root system $\Sigma\left(B_{n}\right)$, the systems in the following diagram are considered to be generalizations of Gauss hypergeometric system and its limits (cf. Section 8). They form a class whose eigenfunctions should be easier to be analyzed than those of other systems in this note.

Hierarchy starting from (Trig- $B C_{n}-$ reg)

$\begin{array}{ccccc} & & & & \text { Toda- } A_{n-1} \\ & & & \nearrow & \\ \text { Rat- } D_{n} & & \text { Trig- } A_{n-1} & \rightarrow & \text { Rat- } A_{n-1} \\ \Uparrow 3: 1 & & \Uparrow_{3: 1} & & \uparrow_{3: 1} \\ \text { Rat- } B_{n-2} & & \text { Trig- } A_{n-1} \text {-bry-reg } & \rightarrow & \text { Rat- } A_{n-1} \text {-bry2 } \\ \uparrow & \nearrow & & & \\ \text { Trig- } B C_{n} \text {-reg } & \rightarrow & \text { Toda- } D_{n} \text {-bry } & \rightarrow & \text { Toda- } B C_{n} \\ \Downarrow_{3: 1} & & \Downarrow_{3: 1} & & \Downarrow_{3: 1} \\ \text { Trig- } D_{n} & \rightarrow & \text { Toda- } D_{n} & \rightarrow & \text { Toda- } A_{n-1} \\ \downarrow & \searrow & & & \\ \text { Trig- } A_{n-1} & & \text { Rat- } D_{n} & & \end{array}$




\subsection{Other forms}

If a Schrödinger operator $P$ is in the commutative algebra $\mathbb{D}=\mathbb{C}\left[P_{1}, \ldots, P_{n}\right]$, then the differential operator $\tilde{P}:=\psi(x)^{-1} P \circ \psi(x)$ with a function $\psi(x)$ is in the commutative algebra $\tilde{\mathbb{D}}=$ $\mathbb{C}\left[\psi(x)^{-1} P_{1} \circ \psi(x), \ldots, \psi(x)^{-1} P_{n} \circ \psi(x)\right]$ of differential operators. Then

$$
\begin{aligned}
& \tilde{P}=-\frac{1}{2} \sum_{j=1}^{n} \frac{\partial^{2}}{\partial x_{j}^{2}}+\sum_{j=1}^{n} a_{j}(x) \frac{\partial}{\partial x_{j}}+\tilde{R}(x), \\
& \frac{\partial \psi(x)}{\partial x_{j}}=a_{j}(x) \quad \text { for } \quad j=1, \ldots, n .
\end{aligned}
$$

Conversely, if a function $\psi(x)$ satisfies (9.12) for a differential operator $\tilde{P}$ of the form (9.11), then $P=\psi(x) \tilde{P} \circ \psi(x)^{-1}$ is of the form (1.1), which we have studied in this note.

If $\psi(x)$ is a function satisfying

$$
\frac{1}{2 \psi(x)} \sum_{j=1}^{n} \frac{\partial^{2} \psi}{\partial x_{j}^{2}}(x)=R(x)
$$

then

$$
\tilde{P}=\psi(x)^{-1}\left(-\frac{1}{2} \sum_{j=1}^{n} \partial_{j}^{2}+R(x)\right) \circ \psi(x)=-\frac{1}{2} \sum_{j=1}^{n} \partial_{j}^{2}-\psi(x)^{-1} \sum_{j=1}^{n} \frac{\partial \psi}{\partial x_{j}}(x) \partial_{j} .
$$

Note that

$$
e^{-\phi(x)} \frac{\partial e^{\phi(x)}}{\partial x_{j}}=\frac{\partial \phi(x)}{\partial x_{j}}, \quad e^{-\phi(x)} \sum_{j=1}^{n} \frac{\partial^{2} e^{\phi(x)}}{\partial x_{j}^{2}}=\sum_{j=1}^{n} \frac{\partial^{2} \phi(x)}{\partial x_{j}^{2}}+\sum_{j=1}^{n}\left(\frac{\partial \phi(x)}{\partial x_{j}}\right)^{2} .
$$

Putting

$$
\phi(x)=m \sum_{1 \leq i<j \leq n} \log \sinh \lambda\left(x_{i}-x_{j}\right),
$$

we have

$$
\begin{aligned}
& \frac{\partial \phi(x)}{\partial x_{k}}=\lambda m \sum_{1 \leq i \leq n, i \neq k} \operatorname{coth} \lambda\left(x_{k}-x_{i}\right) \\
& \sum_{j=1}^{n} \frac{\partial^{2} \phi(x)}{\partial x_{j}^{2}}+\sum_{k=1}^{n}\left(\frac{\partial \phi(x)}{\partial x_{k}}\right)^{2}=-2 \lambda^{2} m \sum_{1 \leq i<j \leq n} \sinh ^{-2} \lambda\left(x_{i}-x_{j}\right) \\
& \quad+2 \lambda^{2} m^{2} \sum_{1 \leq i<j \leq n} \operatorname{coth}^{2} \lambda\left(x_{i}-x_{j}\right)+\lambda^{2} m^{2} \frac{n(n-1)(n-2)}{3} \\
& =2 \lambda^{2} m(m-1) \sum_{1 \leq i<j \leq n} \sinh ^{-2} \lambda\left(x_{i}-x_{j}\right)+\lambda^{2} m^{2} \frac{n\left(n^{2}-1\right)}{3}
\end{aligned}
$$

since

$$
\operatorname{coth} \alpha \cdot \operatorname{coth} \beta+\operatorname{coth} \beta \cdot \operatorname{coth} \gamma+\operatorname{coth} \gamma \cdot \operatorname{coth} \alpha=-1 \quad \text { if } \quad \alpha+\beta+\gamma=0 .
$$

Hence

$$
\tilde{P}=-\frac{1}{2} \sum_{j=1}^{n} \partial_{j}^{2}-m \sum_{1 \leq i<j \leq n} \lambda \operatorname{coth} \lambda\left(x_{i}-x_{j}\right)\left(\partial_{i}-\partial_{j}\right)
$$




$$
\begin{aligned}
& \psi(x)=\prod_{1 \leq i<j \leq n} \lambda^{m} \sinh ^{m} \lambda\left(x_{i}-x_{j}\right), \\
& \psi(x) \circ \tilde{P} \circ \psi^{-1}(x)=-\frac{1}{2} \sum_{j=1}^{n} \partial_{j}^{2}+\sum_{1 \leq i<j \leq n} \frac{m(m-1) \lambda^{2}}{\sinh ^{2} \lambda\left(x_{i}-x_{j}\right)}+\frac{m^{2} n\left(n^{2}-1\right) \lambda^{2}}{6}
\end{aligned}
$$

and $\tilde{P}$ is transformed into the Schrödinger operator of type (Trig- $A_{n-1}$ ).

Now we put

$$
\begin{aligned}
\phi(x)= & m_{0} \sum_{1 \leq i<j \leq n}\left(\log \sinh \lambda\left(x_{i}-x_{j}\right)+\log \sinh \lambda\left(x_{i}+x_{j}\right)\right)+m_{1} \sum_{1 \leq k \leq n} \log \sinh \lambda x_{k} \\
& +m_{2} \sum_{1 \leq k \leq n} \log \sinh 2 \lambda x_{k}
\end{aligned}
$$

and we have

$$
\begin{aligned}
& \frac{\partial \phi(x)}{\partial x_{k}}=\lambda m_{0} \sum_{1 \leq i \leq n, i \neq k}\left(\operatorname{coth} \lambda\left(x_{k}+x_{i}\right)+\operatorname{coth} \lambda\left(x_{k}-x_{i}\right)\right)+\lambda m_{1} \operatorname{coth} \lambda x_{k}+2 \lambda m_{2} \operatorname{coth} 2 \lambda x_{k}, \\
& \operatorname{coth} \lambda x_{k} \operatorname{coth} 2 \lambda x_{k}=1+\frac{1}{2} \sinh ^{-2} \lambda x_{k}, \\
& \sum_{\{i, j, k\}=I}\left(2 \operatorname{coth} \lambda\left(x_{k}+x_{i}\right) \operatorname{coth} \lambda\left(x_{k}-x_{i}\right)+2 \operatorname{coth} \lambda\left(x_{k}+x_{i}\right) \operatorname{coth} \lambda\left(x_{k}-x_{j}\right)\right. \\
& \left.\quad+\operatorname{coth} \lambda\left(x_{k}+x_{i}\right) \operatorname{coth} \lambda\left(x_{k}+x_{j}\right)+\operatorname{coth} \lambda\left(x_{k}-x_{i}\right) \operatorname{coth} \lambda\left(x_{k}-x_{j}\right)\right) \\
& =\sum_{\{i, j, k\}=I}\left(\operatorname{coth} \lambda\left(x_{k}+x_{i}\right) \operatorname{coth} \lambda\left(x_{k}+x_{j}\right)+\operatorname{coth} \lambda\left(x_{i}-x_{j}\right) \operatorname{coth} \lambda\left(x_{i}+x_{k}\right)\right. \\
& \left.+\operatorname{coth} \lambda\left(x_{j}-x_{i}\right) \operatorname{coth} \lambda\left(x_{j}+x_{k}\right)\right)+\sum_{\{i, j, k\}=I}\left(\operatorname{coth} \lambda\left(x_{k}-x_{i}\right) \operatorname{coth} \lambda\left(x_{k}-x_{j}\right)\right)=8
\end{aligned}
$$

for $I \subset\{1, \ldots, n\} \quad$ with $\quad \# I=3$,

$$
\begin{aligned}
& \operatorname{coth} \lambda\left(x_{k}+x_{i}\right)+\operatorname{coth} \lambda\left(x_{k}-x_{i}\right)=\frac{\sinh 2 \lambda x_{k}}{\sinh \lambda\left(x_{k}+x_{i}\right) \sinh \lambda\left(x_{k}-x_{i}\right)}, \\
& \frac{\cosh 2 \lambda x_{k}-\cosh 2 \lambda x_{i}}{\sinh \lambda\left(x_{k}+x_{i}\right) \sinh \lambda\left(x_{k}-x_{i}\right)}=\frac{2 \cosh ^{2} \lambda x_{k}-2 \cosh ^{2} \lambda x_{i}}{\sinh \lambda\left(x_{k}+x_{i}\right) \sinh \lambda\left(x_{k}-x_{i}\right)}=2, \\
& \sum_{k=1}^{n}\left(\frac{\partial \phi(x)}{\partial x_{k}}\right)^{2}=2 \lambda^{2} m_{0}^{2} \sum_{1 \leq i<j \leq n}\left(\operatorname{coth}^{2} \lambda\left(x_{i}-x_{j}\right)+\operatorname{coth}^{2} \lambda\left(x_{i}+x_{j}\right)\right)
\end{aligned}
$$$$
+\lambda^{2} m_{1}^{2} \sum_{k=1}^{n} \operatorname{coth}^{2} \lambda x_{k}+4 \lambda^{2} m_{2}^{2} \sum_{k=1}^{n} \operatorname{coth}^{2} 2 \lambda x_{k}+2 \lambda^{2} m_{1} m_{2} \sum_{k=1}^{n} \sinh ^{-2} \lambda x_{k}
$$$$
+\frac{4 \lambda^{2} m_{0}^{2} n(n-1)(n-2)}{3}+2 \lambda^{2} m_{0}\left(m_{1}+2 m_{2}\right) n(n-1)+4 \lambda^{2} m_{1} m_{2} n,
$$

$$
\begin{aligned}
& \sum_{j=1}^{n} \frac{\partial^{2} \phi(x)}{\partial x_{j}^{2}}+\sum_{k=1}^{n}\left(\frac{\partial \phi(x)}{\partial x_{k}}\right)^{2}=2 \lambda^{2} m_{0}\left(m_{0}-1\right) \sum_{1 \leq i<j \leq n}\left(\sinh ^{-2} \lambda\left(x_{i}-x_{j}\right)+\sinh ^{-2} \lambda\left(x_{i}+x_{j}\right)\right) \\
& +\lambda^{2} m_{1}\left(m_{1}+2 m_{2}-1\right) \sum_{k=1}^{n} \sinh ^{-2} \lambda x_{k}+4 \lambda^{2} m_{2}\left(m_{2}-1\right) \sum_{k=1}^{n} \sinh ^{-2} 2 \lambda x_{k}
\end{aligned}
$$$$
+\lambda^{2}\left(\left(\frac{2}{3} m_{0}(2 n-1)+2 m_{1}+4 m_{2}\right) m_{0}(n-1)+\left(m_{1}+2 m_{2}\right)^{2}\right) n \text {. }
$$ 
Hence

$$
\begin{aligned}
\tilde{P}= & -\frac{1}{2} \sum_{j=1}^{n} \partial_{j}^{2}-\sum_{k=1}^{n} \lambda\left(\sum_{1 \leq i<j \leq n} m_{0}\left(\operatorname{coth} \lambda\left(x_{i}-x_{k}\right)+\operatorname{coth} \lambda\left(x_{i}+x_{k}\right)\right)\right. \\
& \left.+m_{1} \operatorname{coth} \lambda x_{k}+2 m_{2} \operatorname{coth} 2 \lambda x_{k}\right) \partial_{k}, \\
\psi(x) & =\prod_{1 \leq i<j \leq n}\left(\sinh ^{m_{0}} \lambda\left(x_{i}-x_{j}\right) \sinh ^{m_{0}} \lambda\left(x_{i}+x_{j}\right)\right) \prod_{k=1}^{n} \sinh ^{m_{1}} \lambda x_{k} \prod_{k=1}^{n} \sinh ^{m_{2}} 2 \lambda x_{k}
\end{aligned}
$$

and $\tilde{P}$ is transformed into the Schrödinger operator of type (Trig- $B C_{n}$-reg):

$$
\begin{aligned}
\psi(x) \circ \tilde{P} \circ \psi^{-1}(x) & =-\frac{1}{2} \sum_{j=1}^{n} \partial_{j}^{2}+m_{0}\left(m_{0}-1\right) \sum_{1 \leq i<j \leq n}\left(\frac{\lambda^{2}}{\sinh ^{2} \lambda\left(x_{i}-x_{j}\right)}+\frac{\lambda^{2}}{\sinh ^{2} \lambda\left(x_{i}+x_{j}\right)}\right) \\
& +\sum_{k=1}^{n} \frac{m_{1}\left(m_{1}+2 m_{2}-1\right) \lambda^{2}}{2 \sinh ^{2} \lambda x_{k}}+\sum_{k=1}^{n} \frac{2 m_{2}\left(m_{2}-1\right) \lambda^{2}}{\sinh ^{2} 2 \lambda x_{k}} \\
& +\lambda^{2}\left(\frac{m_{0}^{2}}{3}(2 n-1)(n-1)+m_{0}\left(m_{1}+2 m_{2}\right)(n-1)+\frac{\left(m_{1}+2 m_{2}\right)^{2}}{2}\right) n .
\end{aligned}
$$

Remark 23. As is shown in [15, Theorem 5.24 in Ch. II], the operator (9.13) or (9.14) gives the radial part of the differential equation satisfied by the zonal spherical function of a Riemannian symmetric space $G / K$ of the non-compact type which corresponds to the Laplace-Beltrami operator on $G / K$. Here $G$ is a real connected semisimple Lie group with a finite center, $K$ is a maximal compact subgroup of $G$ and the numbers $2 m, 2 m_{0}, 2 m_{1}$ and $2 m_{2}$ correspond to the multiplicities of the roots of the restricted root system for $G$.

Similarly the following operator $\tilde{P}$ is used to characterize the $K$-fixed Whittaker vector $v$ on $G=G L(n, \mathbb{R})$

$$
\begin{aligned}
& \tilde{P}=-\frac{1}{2} \sum_{j=1}^{n} \partial_{j}^{2}+\sum_{j=1}^{n}\left(\frac{n+1}{4}-\frac{j}{2}\right) \partial_{j}+C \sum_{j=1}^{n-1} e^{2\left(x_{j}-x_{j+1}\right)}, \quad \psi(x)=e^{\sum_{j=1}^{n}(j / 2-n+1 / 4) x_{j}}, \\
& \psi(x) \circ \tilde{P} \circ \psi^{-1}(x)=-\frac{1}{2} \sum_{j=1}^{n} \partial_{j}^{2}+C \sum_{j=1}^{n-1} e^{2\left(x_{j}-x_{j+1}\right)}+\frac{n\left(n^{2}-1\right)}{48} .
\end{aligned}
$$

Namely $v$ is a simultaneous eigenfunction of the invariant differential operators on $G / K$ and satisfies $v(n x)=\chi(n) v(x)$ with $n \in N$ and $x \in G / K$. Here $G=K A N$ is an Iwasawa decomposition of $G$ and $\chi$ is a nonsingular character of the nilpotent Lie group $N$. Then $\left.v\right|_{A}$ is a simultaneous eigenfunction of the commuting algebra of differential operators determined by $\tilde{P}$.

\section{References}

[1] Adler M., Some finite-dimensional integrable systems and their behaviour, Comm. Math. Phys. 55 (1977), $195-230$.

[2] Bogoyavlensky O.I., On perturbations of the periodic Toda lattice, Comm. Math. Phys. 51 (1976), 201-209.

[3] Braden H.W., Byatt-Smith J.G.B., On a functional differential equation of determinantal types, Bull. London Math. Soc. 31 (1999), 463-470, math.CA/9804082. 
[4] Buchstaber V.M., Perelomov A.M., On the functional equation related to the quantum three-body problem, in Berezin Memorial Volume, Amer. Math. Soc. Transl. (2) 175 (1996), 15-34, math-ph/0205032.

[5] Calogero F., Solution of the one-dimensional $N$-body problems with quadratic and/or inversely quadratic pair-potentials, J. Math. Phys. 12 (1971), 419-436.

[6] Chalykh O.A., Feigin M., Veselov A., New integrable generalizations of Calogero-Moser quantum problems, J. Math. Phys. 39 (1998), 695-703.

[7] Chalykh O.A., Veselov A., Commutative rings of partial differential operators and Lie algebras, Comm. Math. Phys. 126 (1990), 597-611.

[8] van Diejen J.F., Difference Calogero-Moser systems and finite Toda chains, J. Math. Phys. 36 (1995), 1299-1323.

[9] Goodman R., Wallach N.R., Classical and quantum-mechanical systems of Toda lattice type. I, Comm. Math. Phys. 83 (1982), 355-386.

[10] Goodman R., Wallach N.R., Classical and quantum-mechanical systems of Toda lattice type. III. Joint eigenfunctions of the quantized systems, Comm. Math. Phys. 105 (1986), 473-509.

[11] Heckman G.J., Opdam E.M., Root system and hypergeometric functions. I, Comp. Math. 64 (1987), 329-352.

[12] Inozemtsev V.I., Lax representation with spectral parameter on a torus for integrable particle systems, Lett. Math. Phys. 17 (1989), 11-17.

[13] Inozemtsev V.I., The finite Toda lattices, Comm. Math. Phys. 121 (1989), 629-638.

[14] Levi D., Wojciechowski S., On the Olshanetsky-Perelomov many-body system in an external field, Phys. Lett. A 103 (1984), 11-14.

[15] Helgason S., Groups and geometric analysis, Academic Press, 1984.

[16] Kashiwara M., Oshima T., Systems of differential equations with regular singularities and their boundary value problems, Ann. Math. 106 (1977), 145-200.

[17] Kostant B., The solution to a generalized Toda lattices and representation theory, Adv. Math. 34 (1979), 195-338.

[18] Kuznetsov V.B., Separation of variables for the $D_{n}$-type periodic Toda lattice, J. Phys. A: Math. Gen. 30 (1997), 2127-2138, solv-int/9701009.

[19] Kuznetsov V.B., Jørgensen J., Christiansen P.L., New boundary conditions for integrable lattices, J. Phys. A: Math. Gen. 28 (1995), 4639-4654, hep-th/9503168.

[20] Kuznetsov V.B., Tsyganov A.V., Infinite series of Lie algebras and boundary conditions for integrable systems, J. Soviet Math. 59 (1992), 1085-1092.

[21] Kuznetsov V.B., Tsyganov A.V., Separation of variables for the quantum relativistic Toda lattices, Mathematical Preprint Series, University of Amsterdam, hep-th/9402111.

[22] Moser J., Three integrable Hamiltonian systems connected with isospectral deformation, Adv. Math. 16 (1975), 197-220.

[23] Ochiai H., Commuting differential operators of rank two, Indag. Math. (N.S.) 7 (1996), 243-255.

[24] Ochiai H., Oshima T., Commuting differential operators with $B_{2}$ symmetry, Funkcial. Ekvac. 46 (2003), 297-336.

[25] Ochiai H., Oshima T., Sekiguchi H., Commuting families of symmetric differential operators, Proc. Japan Acad. A 70 (1994), 62-66.

[26] Olshanetsky M.A., Perelomov A.M., Explicit solutions of the periodic Toda lattices, Invent. Math. 54 (1979), 261-269.

[27] Olshanetsky M.A., Perelomov A.M., Classical integrable finite dimensional systems related to Lie algebras, Phys. Rep. 71 (1981), 313-400.

[28] Olshanetsky M.A., Perelomov A.M., Quantum integrable systems related to Lie algebras, Phys. Rep. 94 (1983), 313-404.

[29] Oshima T., Completely integrable systems with a symmetry in coordinates, Asian J. Math. 2 (1998), 935-956, math-ph/0502028.

[30] Oshima T., A class of completely integrable quantum systems associated with classical root systems, Indag. Mathem. 48 (2005), 655-677, math-ph/0502019. 
[31] Oshima T., Commuting differential operators with regular singularities, in Algebraic Analysis of Differential Equations, Springer-Verlag, Tokyo, to appear, math.AP/0611899.

[32] Oshima T., Sekiguchi H., Commuting families of differential operators invariant under the action of a Weyl group, J. Math. Sci. Univ. Tokyo 2 (1995), 1-75.

[33] Ruijsenaars S.N.M., Systems of Calogero-Moser type, Proceedings of the 1994 CRM Banff Summer School 'Particles and Fields', CRM Ser. Math. Phys. (1999), 251-352.

[34] Sekiguchi J., Zonal spherical functions on some symmetric spaces, Publ. Res. Inst. Math. Sci. 12 (1977), suppl., 455-459.

[35] Sergeev A.N., Veselov A.P., Deformed quantum Calogero-Moser problems and Lie superalgebras, Comm. Math. Phys. 245 (2004), 249-278, math-ph/0303025.

[36] Sutherland B., Exact results for a quantum many-body problems in one dimension II, Phys. Rev. A 5 (1972), 1372-1376.

[37] Taniguchi K., On the symmetry of commuting differential operators with singularities along hyperplanes, Int. Math. Res. Not. 36 (2004), 1845-1867, math-ph/0309011.

[38] Toda M., Wave propagation in anharmonic lattice, IPSJ J. 23 (1967), 501-506.

[39] Veselov A., Stykas K.L., Chalykh O.A., Algebraic integrability for the Schrödinger equations and groups generated by reflections, Theor. Math. Phys. 94 (1993), 573-574.

[40] Wakida S., Quantum integrable systems associated with classical Weyl groups, Master Thesis, University of Tokyo, 2004.

[41] Whittaker E.T., Watson G.N., A course of modern analysis, 4th ed., Cambridge University Press, 1927. 